\title{
Fragmentations of Nitrone Triflates to 9-Membered Rings
}

John A. Murphy, ${ }^{\# \star}$ Mohan Mahesh, ${ }^{\#}$ Gary McPheators, ${ }^{\#}$ R. Vijaya Anand, "Thomas M. McGuire, "Robert Carling ${ }^{\mathcal{E}}$ and Alan R. Kennedy"

${ }^{\#}$ WestCHEM, Department of Pure and Applied Chemistry, University of Strathclyde,

295 Cathedral Street, Glasgow G1 1XL, United Kingdom, and and ${ }^{£}$ Merck, Sharp and Dohme Ltd., Neuroscience Research Centre, Terlings Park, Harlow, Essex CM20 2QR, United Kingdom. John.Murphy@strath.ac.uk 
Table of Contents

Section A Experimental Procedures

General Methods and Materials

Spiro [4.5] decan-6-one (18)

2-(2-Bromoethoxy)tetrahydropyran

Preparation of Davis' Oxaziridine (19)

7-(2-Bromoethyl)-7-hydroxyspiro[4.5]decan-6-one (21)

S6

Nitrone (22a)

$2^{\prime}, 3^{\prime}, 5^{\prime}, 6^{\prime}$-Tetrahydrospiro[cyclopentane-1,7'-cyclopenta[b]pyridine]-4'( $\left.1^{\prime} H\right)$-one (23)

$N^{\prime}$-3,4-dihydro-2H-naphthalenylidene- $N, N$-dimethylhydrazine (26)

$N^{\prime}$-[2-Allyl-3,4-dihydro-2H-naphthalenylidene]- $N, N^{\prime}$-dimethylhydrazine (27)

2-Allyl-3,4-dihydro-2H-naphthalen-1-one (28) S8

2-Allyl-2-hydroxy-3,4-dihydro-2H-naphthalen-1-one (29) S9

2-Allyl-2-methoxy-3,4-dihydro-2H-naphthalen-1-one (30) S9

Nitrones (31a) and (31b) S10

3a-(4-Methoxyphenyl)-2-methyl-3,3a,4,5-tetrahydro-2H-benzo[g]indol-2-ol (32) S10

Spiro[4.5]dec-6-ene (36)

S11

7-Oxaspiro[bicyclo[4.1.0]heptane-2,1'-cyclopentane (37)

S12

7-(4-Methoxyphenyl)spiro[4.5]decan-6-ol (38)

7-(4-Methoxyphenyl)spiro[4.5]decan-6-one (39)

7-Allyl-7-(4-methoxyphenyl)spiro[4.5]decan-6-one (40)

-(4-Methoxyphenyl)-6-oxospiro[4.5]dec-7-yl]acetaldehyde (41)

3a'-(4-Methoxyphenyl)-2',3',3a',4',5',6'-hexahydrospiro[cyclopentane-1,7' indole]1'-oxide (43) $\mathbf{S 1 4}$

10-(4-Methoxyphenyl)-7-azaspiro-[4,8]-tridec-10-en-6-one (44)

S15

Fragmentative Rearrangement of Nitrone 46

\section{Section B}

NMR Spectra

Compound $21 \quad{ }^{1} \mathrm{H}$ NMR

Compound $21 \quad{ }^{13} \mathrm{C}$ NMR

Compound 22a ${ }^{1} \mathrm{H}$ NMR

Compound 22a ${ }^{13} \mathrm{C}$ NMR

Compound $23 \quad{ }^{1} \mathrm{H}$ NMR

Compound $23 \quad{ }^{13} \mathrm{C}$ NMR

Compound $26 \quad{ }^{1} \mathrm{H}$ NMR

Compound $26 \quad{ }^{13} \mathrm{C}$ NMR 
$\begin{array}{lll}\text { Compound } 27 & { }^{13} \mathrm{C} \text { NMR } & \text { S26 }\end{array}$

$\begin{array}{llr}\text { Compound } 28 & { }^{1} \mathrm{H} \text { NMR } & \text { S27 }\end{array}$

$\begin{array}{lrr}\text { Compound } 28 & { }^{13} \mathrm{C} \text { NMR } & \text { S28 }\end{array}$

$\begin{array}{llc}\text { Compound } 29 & { }^{1} \text { H NMR } & \text { S29 }\end{array}$

$\begin{array}{lll}\text { Compound } 29 & { }^{13} \mathrm{C} \text { NMR } & \mathbf{S 3 0}\end{array}$

$\begin{array}{lll}\text { Compound } 30 & { }^{1} \mathrm{H} \text { NMR } & \text { S31 }\end{array}$

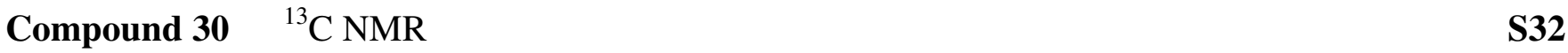

Compound $31 \quad{ }^{1} \mathrm{H}$ NMR (diast 1) $\quad$ S33

$\begin{array}{lll}\text { Compound } 31 & \left.{ }^{13} \mathrm{C} \text { NMR (diast } 1\right) & \mathbf{S 3 4}\end{array}$

$\begin{array}{lll}\text { Compound } 31 & { }^{1} \mathrm{H} \text { NMR (diast 2) } & \mathbf{3 3 5}\end{array}$

$\begin{array}{lll}\text { Compound } 31 & { }^{13} \mathrm{C} \text { NMR (diast 2) } & \mathbf{S 3 6}\end{array}$

$\begin{array}{lll}\text { Compound } 32 & { }^{1} \mathrm{H} \text { NMR } & \text { S37 }\end{array}$

$\begin{array}{llc}\text { Compound } 32 & { }^{13} \mathrm{C} \text { NMR } & \text { S38 }\end{array}$

$\begin{array}{lll}\text { Compound } 38 & { }^{1} \mathrm{H} \text { NMR } & \text { S39 }\end{array}$

$\begin{array}{lll}\text { Compound } 38 & { }^{13} \mathrm{C} \text { NMR } & \mathbf{5 4 0}\end{array}$

$\begin{array}{lll}\text { Compound } 39 & { }^{1} \mathrm{H} \text { NMR } & \mathbf{S 4 1}\end{array}$

$\begin{array}{llr}\text { Compound } 39 & { }^{13} \mathrm{C} \text { NMR } & \mathbf{S 4 2}\end{array}$

$\begin{array}{llr}\text { Compound } 40 & { }^{1} \mathrm{H} \text { NMR } & \mathbf{S 4 3}\end{array}$

$\begin{array}{lll}\text { Compound } 40 & { }^{13} \mathrm{C} \text { NMR } & \text { S44 }\end{array}$

$\begin{array}{llr}\text { Compound } 41 & { }^{1} \mathrm{H} \text { NMR } & \mathbf{S 4 5}\end{array}$

$\begin{array}{lll}\text { Compound } 41 & { }^{13} \mathrm{C} \text { NMR } & \text { S46 }\end{array}$

$\begin{array}{lll}\text { Compound } 43 & { }^{1} \mathrm{H} \text { NMR } & \mathbf{S 4 7}\end{array}$

$\begin{array}{llr}\text { Compound } 43 & { }^{13} \mathrm{C} \text { NMR } & \mathbf{S 4 8}\end{array}$

$\begin{array}{llr}\text { Compound } 44 & { }^{1} \mathrm{H} \text { NMR } & \mathbf{5 4 9}\end{array}$

$\begin{array}{lll}\text { Compound } 44 & { }^{13} \mathrm{C} \text { NMR } & \text { S50 }\end{array}$

$\begin{array}{llc}\text { Compound } 46 & { }^{1} \mathrm{H} \text { NMR } & \text { S51 }\end{array}$

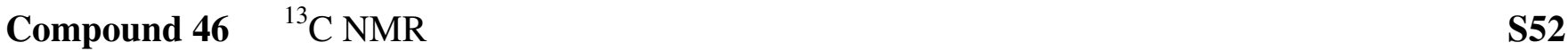

$\begin{array}{lll}\text { Compound } 46 & { }^{1} \mathrm{H}-{ }^{1} \mathrm{H} \text { COSY NMR } & \mathbf{S 5 3}\end{array}$

$\begin{array}{lll}\text { Compound } 47 & { }^{1} \mathrm{H} \text { NMR } & \mathbf{S 5 4}\end{array}$

$\begin{array}{lll}\text { Compound } 47 & { }^{13} \mathrm{C} \text { NMR } & \mathbf{S 5 5}\end{array}$

$\begin{array}{lll}\text { Compound } 47 & { }^{1} \mathrm{H}-{ }^{1} \mathrm{H} \text { COSY NMR } & \mathbf{5 5 6}\end{array}$

$\begin{array}{lll}\text { Compound } 47 & { }^{1} \mathrm{H}-{ }^{13} \mathrm{C} \text { HSQC NMR } & \text { S57 }\end{array}$ 


\section{General methods and Materials:}

Mass spectra were carried out using a QUATTRO instrument, using low resolution electron impact ionization (EI)/chemical ionization (CI) or accurate mass electron impact ionization (EI)/chemical ionization (CI) peak match. Mass spectra were carried out at the EPSRC Mass Spectrometry Service Centre, Swansea. Melting points were measured on a Gallenkamp melting apparatus. Infrared spectra were obtained on a Perkin Elmer Spectrum One FT IR spectrometer. ${ }^{1} \mathrm{H}$ NMR spectra were recorded at $400 \mathrm{MHz}$ on a Bruker DPX 400 or AV400 spectrometer. ${ }^{13} \mathrm{C}$ NMR spectra were recorded at $100.6 \mathrm{MHz}$. Chemical shifts $(\delta)$ are quoted in parts per million (ppm) downfield of tetramethylsilane. Multiplicities are abbreviated as s, singlet; d, doublet; t, triplet; q, quartet; m, multiplet; br, broad. The coupling constants $(J)$ are reported in $\mathrm{Hz}$. Flash chromatography was carried out using Prolabo silica gel 60 (200 - 400 mesh). Thin layer chromatography analysis was carried out on aluminium sheets of silica gel $60 \mathrm{~F}_{254}$. The plates were developed using acidic ethanolic vanillin, potassium permanganate or phosphomolybdic acid solutions.

All reagents were obtained from commercial suppliers and used without further purification unless otherwise stated. Organic solvents were dried and/or distilled prior to use. Petroleum ether was distilled at $40-60^{\circ} \mathrm{C}$; tetrahydrofuran was distilled from sodium and benzophenone; dichloromethane was distilled from calcium hydride; diethyl ether and toluene were distilled over sodium. Unless otherwise stated reactions requiring anhydrous conditions were carried out in dry apparatus under nitrogen.

\section{Spiro [4.5] decan-6-one (18): ${ }^{1}$}

Potassium tert-butoxide $(57.1 \mathrm{~g}, 510 \mathrm{mmol})$ and was stirred in tolueene $(450 \mathrm{~mL})$ were placed in a flamedried flask equipped with a condenser under nitrogen. This was followed by the addition of cyclohexanone $(23.8 \mathrm{~mL}, 230 \mathrm{mmol})$. The reaction mixture was allowed to stir for $1 \mathrm{~h}$ before the addition of 1,4dibromobutane $(27.5 \mathrm{~mL}, 230 \mathrm{mmol})$, after that the reaction was refluxed for $5 \mathrm{~h}$ before cooling to rt. Water $(125 \mathrm{~mL})$ and hydrochloric acid $(15 \% \mathrm{v} / \mathrm{v}, 250 \mathrm{~mL})$ were added to the reaction mixture before extraction with diethyl ether $(2 \times 300 \mathrm{~mL})$. The combined organic layers were washed with saturated aqueous brine solution $(200 \mathrm{~mL})$, dried over anhydrous $\mathrm{Na}_{2} \mathrm{SO}_{4}$, filtered and concentrated under reduced pressure. The mixture was subjected to column chromatography, (98:2 isohexane:EtOAc) to give spiro [4.5] decan-6-one 18 as colorless oil (22.05 g, 63\%); IR (thin film) 2930, 2857, 1707, 1449, 1259, $1124 \mathrm{~cm}^{-1}$; ${ }^{1} \mathrm{H}$ NMR (400 $\left.\mathrm{MHz}, \mathrm{CDCl}_{3}\right) \delta 1.36$ - $1.40\left(\mathrm{~m}, 2 \mathrm{H}, \mathrm{CH}_{2}\right), 1.55$ - $1.59\left(\mathrm{~m}, 4 \mathrm{H}, \mathrm{CH}_{2}\right), 1.69-1.71\left(\mathrm{~m}, 4 \mathrm{H}, \mathrm{CH}_{2}\right), 1.79-1.82$ $\left(\mathrm{m}, 2 \mathrm{H}, \mathrm{CH}_{2}\right), 2.03-2.07\left(\mathrm{~m}, 2 \mathrm{H}, \mathrm{CH}_{2}\right), 2.37-2.40\left(\mathrm{~m}, 2 \mathrm{H}, \mathrm{CH}_{2}\right) ;{ }^{13} \mathrm{C} \mathrm{NMR}\left(100 \mathrm{MHz}, \mathrm{CDCl}_{3}\right) \delta 23.0$ $\left(\mathrm{CH}_{2}\right), 25.4\left(\mathrm{CH}_{2}\right), 27.5\left(\mathrm{CH}_{2}\right), 35.6\left(\mathrm{CH}_{2}\right), 39.6\left(\mathrm{CH}_{2}\right), 40.1\left(\mathrm{CH}_{2}\right), 57.0(\mathrm{~s}), 214.7(\mathrm{~s}) ; \mathrm{m} / \mathrm{z}\left(\mathrm{EI}^{+}\right) 152\left(\mathrm{M}^{+}\right.$, 11\%), 111 (38), 84 (32), 67 (67), 55 (36), 41 (100).

\section{2-(2-Bromoethoxy)tetrahydropyran ${ }^{2}$ - for use in Scheme 4:}

3,4-Dihydro-2H-pyran $(27.3 \mathrm{~mL}, 300 \mathrm{mmol})$ was added to a flame-dried flask containing p-TSA (260 mg, $14.0 \mathrm{mmol})$ and freshly distilled $\mathrm{CH}_{2} \mathrm{Cl}_{2}(175 \mathrm{~mL})$ under nitrogen. The reaction was allowed to stir for 10 min before the dropwise addition of 2-bromoethanol $(14.2 \mathrm{~mL}, 200 \mathrm{mmol})$. The reaction mixture was then 
allowed to stir for additional $18 \mathrm{~h}$ at ambient temperature. Sodium hydrogen carbonate $(1.50 \mathrm{~g})$ was added to the reaction mixture and stirring was continued for a further $50 \mathrm{~min}$ before filtering. The filtrate was evaporated under reduced pressure and the resultant oil was subjected to column chromatography [petroleum ether/ethyl acetate $(90: 10 \mathrm{v} / \mathrm{v})$ ] to give 2-(2-bromoethoxy)tetrahydropyran 29 as a colorless oil [33.4 g (80\%)]; IR (thin film) 2942, 2870, 1441, 1351, 1275, 1200, 1122, 1030, 977, 904, 869, $814 \mathrm{~cm}^{-1} ;{ }^{1} \mathrm{H}$ NMR (400 MHz, $\left.\mathrm{CDCl}_{3}\right) \delta 1.47-1.58\left(\mathrm{~m}, 4 \mathrm{H}, \mathrm{CH}_{2}\right), 1.65-1.71\left(\mathrm{~m}, 1 \mathrm{H}, \mathrm{CH}_{2}\right), 1.73-1.83\left(\mathrm{~m}, 1 \mathrm{H}, \mathrm{CH}_{2}\right)$, 3.41 - $3.50\left(\mathrm{~m}, 3 \mathrm{H}, \mathrm{CH}_{2}, \mathrm{OCH}_{2}\right), 3.70$ - $3.75\left(\mathrm{~m}, 1 \mathrm{H}, \mathrm{OCH}_{2}\right), 3.81$ - $3.86\left(\mathrm{~m}, 1 \mathrm{H}, \mathrm{OCH}_{2}\right), 3.93$ - $3.99(\mathrm{~m}, 1 \mathrm{H}$, $\left.\mathrm{OCH}_{2}\right), 4.60-4.63(\mathrm{~m}, 1 \mathrm{H}, \mathrm{CH}) ;{ }^{13} \mathrm{C} \mathrm{NMR}\left(100 \mathrm{MHz}, \mathrm{CDCl}_{3}\right) \delta 19.3\left(\mathrm{CH}_{2}\right), 25.4\left(\mathrm{CH}_{2}\right), 30.5\left(\mathrm{CH}_{2}\right), 30.9$ $\left(\mathrm{CH}_{2}\right), 62.3\left(\mathrm{CH}_{2}\right), 67.6\left(\mathrm{CH}_{2}\right), 98.9(\mathrm{CH}) ; \mathrm{m} / \mathrm{z}\left(\mathrm{CI}^{+}\right) 226\left(\mathrm{MNH}_{4}^{+}, 94 \%\right) 182(32), 180(25), 102(100)$.

\section{Preparation of Davis' Oxaziridine: ${ }^{3}$ This was prepared in two steps.}

N-Benzylidene-benzenesulfonamide:

Benzenesulfonamide $(15.7 \mathrm{~g}, 100 \mathrm{mmol})$ and dimethoxymethylbenzene $(10.2 \mathrm{~mL}, 100 \mathrm{mmol})$ were placed in a flask connected to a short-path distilling head. The reaction mixture was heated to $150-180{ }^{\circ} \mathrm{C}$, until the entire methanol had been distilled off. The reaction was then placed under high vacuum $(0.1 \mathrm{~mm} \mathrm{Hg})$ until the reaction mixture reached rt. The solid that had formed was dissolved in a small amount of $\mathrm{CH}_{2} \mathrm{Cl}_{2}$; $N$-benzylidenebenzene sulfonamide was precipitated out as a white solid by the slow additional of petroleum ether. Yield $18.9 \mathrm{~g},(77 \%)$; $\mathrm{mp} 82-83{ }^{\circ} \mathrm{C}$ (lit. $\left.{ }^{3} 80^{\circ} \mathrm{C}\right)$; IR ( $\mathrm{KBr}$ disc) 3053, 1597, 1570, 1447, 1313, 1162, 1088, 866, 800, $749 \mathrm{~cm}^{-1} ;{ }^{1} \mathrm{H}$ NMR (400 MHz, $\left.\mathrm{CD}_{3} \mathrm{COCD}_{3}\right) \delta 7.48-7.66(\mathrm{~m}, 6 \mathrm{H}, \mathrm{ArH}), 7.93-8.04$ $(\mathrm{m}, 4 \mathrm{H}, \mathrm{ArH}), 9.08(\mathrm{~s}, 1 \mathrm{H}, \mathrm{CH}) ;{ }^{13} \mathrm{C} \mathrm{NMR}\left(\mathrm{CD}_{3} \mathrm{COCD}_{3}, 100 \mathrm{MHz}\right) \delta 128.6(\mathrm{CH}), 130.0(\mathrm{CH}), 130.1(\mathrm{CH})$, $131.9(\mathrm{CH}), 133.5(\mathrm{C}), 134.4(\mathrm{CH}), 135.8(\mathrm{CH}), 139.5(\mathrm{C}), 171.9(\mathrm{CH}) ; \mathrm{m} / \mathrm{z}\left(\mathrm{CI}^{+}\right) 263\left(\mathrm{MNH}_{4}^{+}, 33 \%\right) 192$ (14), 175 (76), 139 (14), 121 (17), 106 (100).

\section{2-Benzenesulfonyl-3-phenyloxaziridine:}

Saturated aqueous sodium hydrogen carbonate solution $(100 \mathrm{~mL}), N$-benzylidenebenzenesulfonamide (14.7 $\mathrm{g}, 60.0 \mathrm{mmol})$, and BTEAC $(1.37 \mathrm{~g}, 6.0 \mathrm{mmol})$ in chloroform $(100 \mathrm{~mL})$ were placed in a $500 \mathrm{~mL}$, threenecked flask equipped with magnetic stirrer and dropping funnel. The reaction mixture was cooled to $0{ }^{\circ} \mathrm{C}$, with vigorous stirring, a solution of mCPBA $(11.3 \mathrm{~g}, 66.0 \mathrm{mmol})$ in chloroform $(150 \mathrm{~mL})$ was added to the mixture over $30 \mathrm{~min}$ via a dropping funnel. The reaction mixture was allowed to stir for a further $15 \mathrm{~min}$ before the chloroform layer was separated and washed successively with water $(100 \mathrm{~mL}), 10 \%$ saturated aqueous sodium sulfite $(100 \mathrm{~mL})$, water $(2 \times 100 \mathrm{~mL})$ and finally with saturated aqueous brine solution $(50$ $\mathrm{mL}$ ). The chloroform layer was then dried over anhydrous magnesium sulfate and filtered, before being concentrated under reduced pressure at a temperature that did not exceed $40{ }^{\circ} \mathrm{C}$ to give 2-benzensulfonyl-3phenyloxaziridine as a colorless powder (11.7 g, 76\%); mp 96-97 ${ }^{\circ} \mathrm{C}$; (lit. $\left.{ }^{3} 96-98{ }^{\circ} \mathrm{C}\right)$; IR ( $\mathrm{KBr}$ disc) 3063 , 1574, 1446, 1350, 1168, 760, $728 \mathrm{~cm}^{-1}$; ${ }^{1} \mathrm{H}$ NMR (400 MHz, $\left.\mathrm{CDCl}_{3}\right) \delta 5.50(\mathrm{~s}, 1 \mathrm{H}, \mathrm{CH}), 7.40-7.48(\mathrm{~m}, 5 \mathrm{H}$, ArH), 7.63 - 7.67 (m, 2H, ArH), $7.75-7.79$ (m, 1H, ArH), 8.06 - 8.08 (m, 2H, ArH); ${ }^{13} \mathrm{C} \mathrm{NMR} \mathrm{(100} \mathrm{MHz,}$ 
$\left.\mathrm{CDCl}_{3}\right) \delta 76.5(\mathrm{CH}), 126.7(\mathrm{CH}), 128.5(\mathrm{CH}), 129.0(\mathrm{CH}), 129.2(\mathrm{CH})$, 129,4 (CH), $129.5(\mathrm{CH}), 129.6(\mathrm{CH})$, $129.9(\mathrm{CH}), 130.7(\mathrm{C}), 131.7(\mathrm{CH}), 135.0(\mathrm{C}), 135.2(\mathrm{CH})$.

\section{7-(2-Bromoethyl)-7-hydroxyspiro[4.5]decan-6-one (21):}

Potassium tert-butoxide (1.44 g, $12.9 \mathrm{mmol})$ was placed in a flame-dried flask containing a magnetic stirrer under nitrogen. Freshly distilled benzene $(25 \mathrm{~mL})$ and spiroketone 18 (1.65 g, $10.8 \mathrm{mmol})$ were then added to the flask. The reaction mixture was allowed to stir for $15 \mathrm{~min}$ before the slow addition of 2-(2bromoethoxy)tetrahydropyran $(4.5 \mathrm{~g}, 21.4 \mathrm{mmol})$. At this point, the reaction vessel was placed in an oil bath and allowed to reflux for $18 \mathrm{~h}$. It was then allowed to cool to rt before the addition of water (200 $\mathrm{mL})$ and then hydrochloric acid $(15 \% \mathrm{v} / \mathrm{v}, 250 \mathrm{~mL})$. It was then extracted with diethyl ether $(2 \times 300 \mathrm{~mL})$. The combined organic phases were washed sequentially with saturated aqueous sodium hydrogen carbonate solution $(300 \mathrm{~mL})$ and brine $(300 \mathrm{~mL})$ before drying over anhydrous sodium sulfate, filtering and concentrating under reduced pressure. Column chromatography, [petroleum ether/ethyl acetate (95:5 v/v)], gave 7-[2-tetrahydropyran-2-yloxy)ethyl]spiro[4.5] decan-6-one, as a pair of diastereomers, and as a colorless oil (300 mg), which was used without further purification

Freshly distilled THF (10 mL) and KHMDS (310 mg, $1.56 \mathrm{mmol})$ were added to a flame-dried flask purged with argon and equipped with magnetic stirrer. The temperature of the reaction was lowered to $-78{ }^{\circ} \mathrm{C}$ before the addition of 7-[2-tetrahydropyran-2-yloxy)ethyl]spiro[4.5] decan-6-one (280 $\mathrm{mg}, 1.0 \mathrm{mmol}$ ) dissolved in anhydrous THF $(3 \mathrm{~mL})$. The reaction was allowed to stir for $30 \mathrm{~min}$ before the addition of the Davis oxaziridine 19 (380 mg, $1.56 \mathrm{mmol})$ dissolved in THF ( $3 \mathrm{~mL})$. The reaction mixture was then stirred for a further $20 \mathrm{~min}$ at $-78^{\circ} \mathrm{C}$ before the reaction was allowed to reach $\mathrm{rt}$ and stirred for $18 \mathrm{~h}$. Saturated aqueous ammonium chloride solution $(25 \mathrm{~mL})$ was used to quench the reaction. It was then extracted with diethyl ether ( $3 \times 20 \mathrm{~mL})$, before being separated and concentrated under reduced pressure to give a sticky precipitate. The precipitate was filtered using a 50/50 mixture of chloroform and petroleum ether, the filtrate was collected and concentrated under reduced pressure to give a viscous oil, which was then subjected to column chromatography, (ethyl acetate:petroleum ether:: 15:85), to give 7-hydroxy-7-[2(tetrahydropyran-2-yloxy)ethyl]spiro[4.5]decan-6-one 20, which exists as a pair of diastereoisomers, as a colorless oil (132 mg), which was carried forward for the next stage.

Triphenylphosphine $(167 \mathrm{mg}, 0.64 \mathrm{mmol})$ was added to a flame-dried flask containing freshly distilled $\mathrm{CH}_{2} \mathrm{Cl}_{2}(5 \mathrm{~mL})$. The reaction temperature was reduced to $0{ }^{\circ} \mathrm{C}$, before the dropwise addition of bromine (100 mg, $0.64 \mathrm{mmol})$. After being stirred for $30 \mathrm{~min}$ at $0{ }^{\circ} \mathrm{C}$, compound 20 (130 mg, $0.45 \mathrm{mmol}$ ) was added. The reaction was allowed to rise in ambient temperature before being stirred for $12 \mathrm{~h}$. The solvent was then removed under reduced pressure and the residue purified by column chromatography [petroleum ether/ethyl acetate (85:15 v/v)] to give 7-(2-bromethyl)-7-hydroxyspiro[4.5] decan-6-one 21 as a colorless oil. Yield 95 mg (3.2 \% from 18); [Found (ES): $\mathrm{M}^{+}, 274.0562, \mathrm{C}_{12} \mathrm{H}_{19} \mathrm{BrO}_{2}$ requires $M, 274.0563$ ]; IR (thin film) 3477 , 2940, 2868, 1693, 1448, 1025, $866 \mathrm{~cm}^{-1} ;{ }^{1} \mathrm{H}$ NMR (400 MHz, $\left.\mathrm{CDCl}_{3}\right) \delta 1.21-1.26\left(\mathrm{~m}, 1 \mathrm{H}, \mathrm{CH}_{2}\right), 1.54$ $1.90\left(\mathrm{~m}, 11 \mathrm{H}, \mathrm{CH}_{2}\right), 2.16-2.20\left(\mathrm{~m}, 1 \mathrm{H}, \mathrm{CH}_{2}\right), 2.28-2.32\left(\mathrm{~m}, 2 \mathrm{H}, \mathrm{CH}_{2}\right), 2.46-2.49\left(\mathrm{~m}, 1 \mathrm{H}, \mathrm{CH}_{2}\right), 3.10-$ 
$3.16\left(\mathrm{~m}, 1 \mathrm{H}, \mathrm{CH}_{2}\right), 3.44-3.48\left(\mathrm{~m}, 1 \mathrm{H}, \mathrm{CH}_{2}\right), 4.10$ (brs, $\left.1 \mathrm{H}, \mathrm{OH}\right) ;{ }^{13} \mathrm{C} \mathrm{NMR}\left(100 \mathrm{MHz}, \mathrm{CDCl}_{3}\right) \delta 19.6$ $\left(\mathrm{CH}_{2}\right), 24.8\left(\mathrm{CH}_{2}\right), 26.0\left(\mathrm{CH}_{2}\right), 27.0\left(\mathrm{CH}_{2}\right), 35.9\left(\mathrm{CH}_{2}\right), 37.0\left(\mathrm{CH}_{2}\right), 39.4\left(\mathrm{CH}_{2}\right), 39.5\left(\mathrm{CH}_{2}\right), 41.7\left(\mathrm{CH}_{2}\right)$, 55.6 (C), 78.9 (C), 215.9 (C); m/z (EI $) 274\left(\mathrm{M}^{+}, 63 \%\right)$, 259, (63), 257 (62), 195 (38), 121, (43), 94 (76), 67 (100), 55 (94), 41 (88).

\section{Nitrone (22a):}

Ethanol $(15 \mathrm{~mL})$ and water $(1 \mathrm{~mL})$ were added to a 3-necked flask equipped with condenser, followed by hydroxylamine hydrochloride (298 mg, $4.3 \mathrm{mmol}$ ), sodium hydroxide (172 mg, $4.3 \mathrm{mmol}$ ), alcohol 21 (600 $\mathrm{mg}, 2.15 \mathrm{mmol})$ and potassium carbonate $(297 \mathrm{mg}, 2.15 \mathrm{mmol})$. The reaction mixture was then refluxed for $6 \mathrm{~h}$ and then cooled to rt. The solvent was removed under reduced pressure to give a residue that was purified by column chromatography (DCM:methanol :: 20:1) to give nitrone 22a as white solid. Yield 144 mg (32\%); mp 110-112 ${ }^{\circ} \mathrm{C}$; [Found (ES): $\mathrm{MH}^{+}, 210.1493 . \mathrm{C}_{12} \mathrm{H}_{20} \mathrm{NO}_{2}(\mathrm{MH})$ requires $M H, 210.1489$ ]; FT IR (KBr disc) 3270, 2947, 2862, 1589, 1444, 1361, 1298, 1215, 1135, 957, 938, 799, $611 \mathrm{~cm}^{-1}$; ${ }^{1} \mathrm{H}$ NMR (400 $\left.\mathrm{MHz}, \mathrm{CDCl}_{3}\right) \delta 1.12-1.14\left(\mathrm{~m}, 1 \mathrm{H}, \mathrm{CH}_{2}\right), 1.28-1.53\left(\mathrm{~m}, 4 \mathrm{H}, \mathrm{CH}_{2}\right), 1.66-1.86\left(\mathrm{~m}, 6 \mathrm{H}, \mathrm{CH}_{2}\right), 1.98-2.03$ (m, 1H, $\left.\mathrm{CH}_{2}\right), 2.07$ - $2.14\left(\mathrm{~m}, 2 \mathrm{H}, \mathrm{CH}_{2}\right), 3.13$ - $3.21\left(\mathrm{~m}, 2 \mathrm{H}, \mathrm{CH}_{2}\right), 3.70$ - $3.76\left(\mathrm{~m}, 1 \mathrm{H}, \mathrm{CH}_{2}\right), 4.19$ - $4.26(\mathrm{~m}$, $\left.1 \mathrm{H}, \mathrm{CH}_{2}\right), 4.88$, (brs, $\left.1 \mathrm{H}, \mathrm{OH}\right) ;{ }^{13} \mathrm{C}$ NMR $\left(100 \mathrm{MHz}, \mathrm{CDCl}_{3}\right) \delta 18.7\left(\mathrm{CH}_{2}\right), 25.7\left(\mathrm{CH}_{2}\right), 26.0\left(\mathrm{CH}_{2}\right), 34.7$ $\left(\mathrm{CH}_{2}\right), 34.8\left(\mathrm{CH}_{2}\right), 37.2\left(\mathrm{CH}_{2}\right), 38.3\left(\mathrm{CH}_{2}\right), 38.3\left(\mathrm{CH}_{2}\right), 50.5(\mathrm{C}), 61.5\left(\mathrm{CH}_{2}\right), 79.9(\mathrm{C}), 153.6(\mathrm{C}) ; \mathrm{m} / \mathrm{z}\left(\mathrm{EI}^{+}\right)$ $209\left(\mathrm{M}^{+}, 25 \%\right)$, 192, (33), 174 (42), 168 (100), 150 (85), 91 (92), 79 (90), 77 (87),67 (79).

\section{2',3',5',6'-Tetrahydrospiro[cyclopentane-1,7'-cyclopenta[b]pyridine]-4'(1'H)-one (23):}

Nitrone 22a (170 mg, $0.81 \mathrm{mmol})$ was dissolved in $\mathrm{CH}_{2} \mathrm{Cl}_{2}(10 \mathrm{~mL})$. Trifluoromethanesulfonic anhydride ( $251 \mathrm{mg}, 0.89 \mathrm{mmol}$ ) was then added. The reaction was stirred for $2 \mathrm{~h}$ before the addition of water. $\mathrm{CH}_{2} \mathrm{Cl}_{2}$ $(3 \times 10 \mathrm{~mL})$ was used to extract the reaction. The combined organic extracts were washed with brine before drying over anhydrous sodium sulfate, filtering and concentrating under reduced pressure. HPLC $\left[\mathrm{CH}_{2} \mathrm{Cl}_{2} /\right.$ methanol $\left.(99: 1 \mathrm{v} / \mathrm{v})\right]$ was used to isolate $2^{\prime}, 3^{\prime}, 5^{\prime}, 6^{\prime}$-Tetrahydrospiro[cyclopentane-1,7'cyclopenta[b]pyridine]-4'(1'H)-one $\mathbf{2 3}$ as a brown oil. Yield $71 \mathrm{mg}(46 \%)$; [Found (ES): $\mathrm{MH}^{+}, 192.1383$, $\mathrm{C}_{12} \mathrm{H}_{18} \mathrm{NO}(\mathrm{MH})$ requires $\left.M H, 192.1383\right]$; FT IR (thin film) 3221, 3070, 2950, 2863, 1619, 1548, 1447, $1419,1342,1318,1266,1227,1176,895,680 \mathrm{~cm}^{-1} ;{ }^{1} \mathrm{H}$ NMR $\left(400 \mathrm{MHz}, \mathrm{CDCl}_{3}\right) \delta 1.49-1.86(\mathrm{~m}, 10 \mathrm{H}$, $\left.\mathrm{CH}_{2}\right), 2.41-2.47$ (m, 4H, $\left.\mathrm{CH}_{2}\right), 3.55-3.59$ (m, 2H, $\left.\mathrm{CH}_{2}\right), 4.64$ (brs, $\left.1 \mathrm{H}, \mathrm{NH}\right) ;{ }^{13} \mathrm{C} \mathrm{NMR}\left(100 \mathrm{MHz}, \mathrm{CDCl}_{3}\right)$ $\delta 24.9\left(\mathrm{CH}_{2}\right), 25.8\left(\mathrm{CH}_{2}\right), 36.4\left(\mathrm{CH}_{2}\right), 37.5\left(\mathrm{CH}_{2}\right), 38.3\left(\mathrm{CH}_{2}\right), 43.9\left(\mathrm{CH}_{2}\right) 56.2(\mathrm{C}), 109.7(\mathrm{C}), 172.9(\mathrm{C})$, $189.9(\mathrm{C}) ; \mathrm{m} / \mathrm{z}\left(\mathrm{EI}^{+}\right) 191\left(\mathrm{M}^{+}, 83 \%\right), 176$ (32), 162 (44), 148 (100), 134 (37), 122 (55), 106 (42), 94 (65).

\section{$N^{\prime}$-3,4-dihydro-2H-naphthalenylidene- $N, N$-dimethylhydrazine (26):}

A mixture of $\alpha$-tetralone $25(2.73 \mathrm{~mL}, 20.5 \mathrm{mmol}), N, N$-dimethylhydrazine $(4.7 \mathrm{~mL}, 61.6 \mathrm{mmol})$ and acetic acid ( a few drops) in absolute ethanol $(5 \mathrm{~mL})$ was refluxed for $24 \mathrm{~h}$. The volatile constituents were removed under reduced pressure and the crude on purification over a short pack of basic alumina column using 5\% EtOAc in petroleum ether gave $N^{\prime}$-3,4-dihydro-2H-naphthalenylidene- $N, N$-dimethylhydrazine $\mathbf{2 6}$ as yellow 
oil (3.4 g, 88\%); [Found (ES): $\mathrm{MH}^{+}, 189.1387, \mathrm{C}_{12} \mathrm{H}_{17} \mathrm{~N}_{2}(\mathrm{MH})$ requires $M H, 189.1386$ ]; IR (thin film) 2949, 2858, 1612, 1466, 1451, $979 \mathrm{~cm}^{-1} ;{ }^{1} \mathrm{H}$ NMR (400 MHz, $\left.\mathrm{CDCl}_{3}\right) \delta 1.89-1.96\left(\mathrm{~m}, 2 \mathrm{H}, \mathrm{CH}_{2}\right), 2.61(\mathrm{~s}$, $\left.6 \mathrm{H}, \mathrm{CH}_{3}\right), 2.80-2.86\left(\mathrm{~m}, 4 \mathrm{H}, \mathrm{CH}_{2}\right), 7.13(\mathrm{~d}, \mathrm{~J}=7.5 \mathrm{~Hz}, 1 \mathrm{H}, \mathrm{ArH}), 7.20(\mathrm{dd}, \mathrm{J}=7.9,6.7 \mathrm{~Hz}, 1 \mathrm{H}, \operatorname{ArH}), 7.27$ (ddd, J = 7.5, 6.7, $1.4 \mathrm{~Hz}, 1 \mathrm{H}, \mathrm{ArH}), 8.16(\mathrm{~d}, \mathrm{~J}=7.9,1 \mathrm{H}, \mathrm{ArH}) ;{ }^{13} \mathrm{C} \mathrm{NMR}\left(100 \mathrm{MHz}, \mathrm{CDCl}_{3}\right) \delta 22.8\left(\mathrm{CH}_{2}\right)$, $27.5\left(\mathrm{CH}_{2}\right), 30.0\left(\mathrm{CH}_{2}\right), 47.5\left(\mathrm{CH}_{3}\right), 125.2(\mathrm{CH}), 126.3(\mathrm{CH}), 128.8(\mathrm{CH}), 129.5(\mathrm{CH}), 133.2(\mathrm{C}), 139.9(\mathrm{C})$, $161.2(\mathrm{C}) ; m / z\left(\mathrm{CI}^{+}\right) 189\left(\mathrm{MH}^{+}, 100 \%\right), 146(60), 100(30)$.

\section{$N^{\prime}$-[2-Allyl-3,4-dihydro-2H-naphthalenylidene]- $N$, $N^{\prime}$-dimethylhydrazine (27):}

$n$-Butyllithium (2.5 M solution in THF) $(2.34 \mathrm{~mL}, 5.8 \mathrm{mmol})$ was added dropwise to a solution of $N, N$ diisopropyl amine $(820 \mu \mathrm{L}, 5.8 \mathrm{mmol})$ in anhydrous $\mathrm{THF}(20 \mathrm{~mL})$ at $0{ }^{\circ} \mathrm{C}$. After being stirred for $15 \mathrm{~min}, 36$ $(1.0 \mathrm{~g}, 5.3 \mathrm{mmol})$ in anhydrous THF $(5 \mathrm{~mL})$ was added to it in a dropwise manner and the resulting solution was stirred at $0{ }^{\circ} \mathrm{C}$ for $1 \mathrm{~h}$. Allyl bromide $(460 \mu \mathrm{L}, 5.3 \mathrm{mmol})$ in anhydrous THF $(5 \mathrm{~mL})$ was then added dropwise to it and the reaction mixture was slowly warmed to room temperature and stirred for additional $30 \mathrm{~h}$. It was quenched by the addition of saturated aqueous $\mathrm{NH}_{4} \mathrm{Cl}$ solution and extracted with EtOAc (20 $\mathrm{mL}$ x 3). The combined organic layer was washed with saturated brine solution and dried over anhydrous $\mathrm{Na}_{2} \mathrm{SO}_{4}$. Evaporation of the solvent followed by purification over a short pack of basic alumina column using 5\% EtOAc in petroleum ether gave $N^{\prime}$-[2-allyl-3,4-dihydro-2H-naphthalenylidene]- $N, N^{\prime}$ dimethylhydrazine 27 as a low melting, pale yellow solid (1.05 g, 87\%); mp $28-34{ }^{\circ} \mathrm{C}$; [Found (ES): $\mathrm{MH}^{+}$, 229.1703, $\mathrm{C}_{15} \mathrm{H}_{21} \mathrm{~N}_{2}(\mathrm{MH})$ requires $\left.M H, 229.1699\right]$ ] IR $\left(\mathrm{CH}_{2} \mathrm{Cl}_{2}\right)$ 2947, 2853, 1639, 1615, 1466, 1449, 968 $\mathrm{cm}^{-1} ;{ }^{1} \mathrm{H}$ NMR $\left(400 \mathrm{MHz}, \mathrm{CDCl}_{3}\right) \delta 1.92-2.01\left(\mathrm{~m}, 1 \mathrm{H}, \mathrm{CH}_{2}\right), 2.09-2.14\left(\mathrm{~m}, 1 \mathrm{H}, \mathrm{CH}_{2}\right), 2.22-2.37(\mathrm{~m}$, $\left.2 \mathrm{H}, \mathrm{CH}_{2}\right), 2.62\left(\mathrm{~s}, 6 \mathrm{H}, \mathrm{CH}_{3}\right), 2.78-2.83\left(\mathrm{~m}, 1 \mathrm{H}, \mathrm{CH}_{2}\right), 3.01-3.10\left(\mathrm{~m}, 1 \mathrm{H}, \mathrm{CH}_{2}\right), 3.77-3.79(\mathrm{bm}, 1 \mathrm{H}, \mathrm{CH})$, $5.11-5.14\left(\mathrm{~m}, 2 \mathrm{H}, \mathrm{CH}_{2}=\right), 5.87-5.97(\mathrm{~m}, 1 \mathrm{H}, \mathrm{CH}=), 7.20(\mathrm{~d}, J=7.5 \mathrm{~Hz}, 1 \mathrm{H}, \mathrm{ArH}), 7.27(\mathrm{t}, J=7.5 \mathrm{~Hz}, 1 \mathrm{H}$, ArH), $7.34-7.38(\mathrm{~m}, 1 \mathrm{H}, \mathrm{ArH}), 8.16(\mathrm{~d}, J=7.8 \mathrm{~Hz}, 1 \mathrm{H}, \mathrm{ArH}) ;{ }^{13} \mathrm{C} \mathrm{NMR}\left(100 \mathrm{MHz}, \mathrm{CDCl}_{3}\right) \delta 24.5\left(\mathrm{CH}_{2}\right)$, $25.17\left(\mathrm{CH}_{2}\right), 33.9(\mathrm{CH}), 34.1\left(\mathrm{CH}_{2}\right), 48.3\left(\mathrm{CH}_{3}\right), 116.5\left(\mathrm{CH}_{2}\right), 126.2(\mathrm{CH}), 126.4(\mathrm{CH}), 128.9(\mathrm{CH}), 129.8$ $(\mathrm{CH}), 136.7(\mathrm{CH}), 139.1(\mathrm{C}) ; \mathrm{m} / z\left(\mathrm{CI}^{+}\right) 229\left(\mathrm{MH}^{+}, 100 \%\right), 186(62), 146(32)$.

\section{2-Allyl-3,4-dihydro-2H-naphthalen-1-one (28):}

A solution of $2 \mathrm{~N}$ aqueous $\mathrm{HCl}(10 \mathrm{~mL})$ was added to a solution of allylated hydrazone $27(1.30 \mathrm{~g}, 5.7 \mathrm{mmol})$ in $\mathrm{MeOH}(40 \mathrm{~mL})$ at room temperature and stirred vigorously for $4 \mathrm{~h}$. $\mathrm{MeOH}$ was removed under reduced pressure and the aqueous layer was extracted with EtOAc $(15 \mathrm{~mL}$ x 2). The combined organic layer was washed with saturated aqueous sodium bicarbonate solution followed by saturated brine solution and dried over anhydrous $\mathrm{Na}_{2} \mathrm{SO}_{4}$. Evaporation of the solvent followed by purification over silica gel column $(5 \%$ EtOAc in petroleum ether) gave 2-allyl-3,4-dihydro-2H-naphthalen-1-one 28 as a colorless oil (760 mg, 72\%); [Found (ES): $\mathrm{MH}^{+}, 187.1116, \mathrm{C}_{13} \mathrm{H}_{15} \mathrm{O}(\mathrm{MH})$ requires $M H, 187.1117$ ]; IR (thin film) 1683, 1640, $1600 \mathrm{~cm}^{-1} ;{ }^{1} \mathrm{H}$ NMR $\left(400 \mathrm{MHz}, \mathrm{CDCl}_{3}\right) \delta 1.84-1.95\left(\mathrm{~m}, 1 \mathrm{H}, \mathrm{CH}_{2}\right), 2.22-2.34\left(\mathrm{~m}, 2 \mathrm{H}, \mathrm{CH}_{2}\right), 2.54-2.61$ $\left(\mathrm{m}, 1 \mathrm{H}, \mathrm{CH}_{2}\right), 2.76-2.82(\mathrm{~m}, 1 \mathrm{H}, \mathrm{CH}), 3.02\left(\mathrm{dd}, J=7.6,4.5 \mathrm{~Hz}, 2 \mathrm{H}, \mathrm{CH}_{2}\right), 5.09-5.17\left(\mathrm{~m}, 2 \mathrm{H}, \mathrm{CH}_{2}=\right)$, 
$5.83-5.94(\mathrm{~m}, 1 \mathrm{H}, \mathrm{CH}=), 7.26(\mathrm{~d}, 1 \mathrm{H}, \mathrm{J}=7.6 \mathrm{~Hz}, \mathrm{ArH}), 7.29-7.34(\mathrm{~m}, 1 \mathrm{H}, \mathrm{ArH}), 7.48(\mathrm{td}, \mathrm{J}=7.6,1.1 \mathrm{~Hz}$, $1 \mathrm{H}, \mathrm{ArH}), 8.06(\mathrm{dd}, \mathrm{J}=7.8,1.1 \mathrm{~Hz}, 1 \mathrm{H}, \mathrm{ArH}) ;{ }^{13} \mathrm{C} \mathrm{NMR}\left(100 \mathrm{MHz}, \mathrm{CDCl}_{3}\right) \delta 28.1\left(\mathrm{CH}_{2}\right), 28.9\left(\mathrm{CH}_{2}\right)$, $34.23\left(\mathrm{CH}_{2}\right), 47.3(\mathrm{CH}), 117.0\left(\mathrm{CH}_{2}\right), 126.7(\mathrm{CH}), 127.6(\mathrm{CH}), 128.9(\mathrm{CH}), 132.7(\mathrm{C}), 133.4(\mathrm{CH}), 136.4$ (CH), $144.2(\mathrm{C}), 199.5(\mathrm{C}) ; \mathrm{m} / z\left(\mathrm{CI}^{+}\right) 204\left(\mathrm{MNH}_{4}{ }^{+}, 48 \%\right), 187$ (100).

\section{2-Allyl-2-hydroxy-3,4-dihydro-2H-naphthalen-1-one (29):}

Potassium bis(trimethylsilyl) amide (0.5 M solution in toluene) ( $8.60 \mathrm{~mL}, 4.3 \mathrm{mmol}$ ) was added drop-wise to a solution of allyl tetralone $28(760 \mathrm{mg}, 4.1 \mathrm{mmol})$ in anhydrous THF $(20 \mathrm{~mL})$ at $-78{ }^{\circ} \mathrm{C}$. After being stirred for half an hour, a solution of Davis' oxaziridine (1.07 g, $4.3 \mathrm{mmol})$ in anhydrous THF (20 mL) was added dropwise to it. The resulting solution was then slowly warmed to room temperature and stirred for 20 h. It was quenched with saturated aqueous $\mathrm{NH}_{4} \mathrm{Cl}$ solution and extracted with EtOAc (20 mL x 3). The combined organic layer was washed with saturated brine solution and dried over anhydrous $\mathrm{Na}_{2} \mathrm{SO}_{4}$. Evaporation of the solvent followed by purification over silica gel column [20\% EtOAc in petroleum ether] gave 2-allyl-2-hydroxy-3,4-dihydro-2H-naphthalen-1-one 29 as a colorless oil (480 mg, 58\%); [Found (ES): $\mathrm{MNH}_{4}{ }^{+}, 220.1329, \mathrm{C}_{13} \mathrm{H}_{18} \mathrm{O}_{2} \mathrm{~N}\left(\mathrm{MNH}_{4}\right)$ requires $\left.M N H_{4}, 220.1332\right]$; IR (thin film) 3486, 1683, 1639, 1602 $\mathrm{cm}^{-1} ;{ }^{1} \mathrm{H}$ NMR $\left(400 \mathrm{MHz}, \mathrm{CDCl}_{3}\right) \delta 2.17\left(\mathrm{td}, \mathrm{J}=12.9,5.7 \mathrm{~Hz}, 1 \mathrm{H}, \mathrm{CH}_{2}\right), 2.33-2.39\left(\mathrm{~m}, 2 \mathrm{H}, \mathrm{CH}_{2}\right), 2.45(\mathrm{dd}$, $\left.\mathrm{J}=14.1,6.1 \mathrm{~Hz}, 1 \mathrm{H}, \mathrm{CH}_{2}\right), 2.99\left(\mathrm{dd}, \mathrm{J}=17.8,5.6 \mathrm{~Hz}, 1 \mathrm{H}, \mathrm{CH}_{2}\right), 3.06-3.15\left(\mathrm{~m}, 1 \mathrm{H}, \mathrm{CH}_{2}\right), 3.66(\mathrm{bs}, 1 \mathrm{H}$, $\mathrm{OH}$ ), 5.09 (ddm, J = 17.1, 2.96, Hz, 1H, $\left.\mathrm{CH}_{2}=\right), 5.17$ (dd, J = 10.1, 0.8 Hz, CH=), $5.83-5.93$ (m, 1H, CH=), $7.26(\mathrm{~d}, \mathrm{~J}=7.6 \mathrm{~Hz}, 1 \mathrm{H}, \mathrm{ArH}), 7.34(\mathrm{t}, \mathrm{J}=7.6 \mathrm{~Hz}, 1 \mathrm{H}, \mathrm{ArH}), 7.5(\mathrm{td}, \mathrm{J}=7.5,1.4 \mathrm{~Hz}, 1 \mathrm{H}, \mathrm{ArH}), 8.01(\mathrm{~d}, \mathrm{~J}=$ $7.8 \mathrm{~Hz}, 1 \mathrm{H}, \mathrm{ArH}) ;{ }^{13} \mathrm{C}$ NMR (100 MHz, $\left.\mathrm{CDCl}_{3}\right) \delta 26.4\left(\mathrm{CH}_{2}\right), 33.73\left(\mathrm{CH}_{2}\right), 40.5\left(\mathrm{CH}_{2}\right), 75.6(\mathrm{C}), 119.4$ $\left(\mathrm{CH}_{2}\right), 127.2(\mathrm{CH}), 128.2(\mathrm{CH}), 129.1(\mathrm{CH}), 130.4(\mathrm{C}), 132.3(\mathrm{CH}), 134.3(\mathrm{CH}), 143.6(\mathrm{C}), 201.2(\mathrm{C}) ; \mathrm{m} / \mathrm{z}$ $\left(\mathrm{CI}^{+}\right) 220\left(\mathrm{MNH}_{4}^{+}, 100 \%\right), 204$ (52), 187 (84), $146(48)$.

\section{2-Allyl-2-methoxy-3,4-dihydro-2H-naphthalen-1-one (30):}

A solution of hydroxyketone $29(1.0 \mathrm{~g}, 4.95 \mathrm{mmol})$ in anhydrous THF (5 mL) was added dropwise to a suspension of sodium hydride [60\% suspension in mineral oil] (240 mg, $5.9 \mathrm{mmol})$ in anhydrous THF (10 $\mathrm{mL})$ at $0{ }^{\circ} \mathrm{C}$. After being stirred for $1 \mathrm{~h}$, dimethyl sulfate $(700 \mu \mathrm{L}, 7.4 \mathrm{mmol})$ was slowly added to it. The resulting solution was then slowly warmed to room temperature and stirred for $24 \mathrm{~h}$. It was quenched with saturated aqueous $\mathrm{NH}_{4} \mathrm{Cl}$ solution and extracted with diethyl ether $(20 \mathrm{~mL}$ x 3). The combined organic layer was washed with saturated brine solution and dried over anhydrous $\mathrm{Na}_{2} \mathrm{SO}_{4}$. Evaporation of the solvent followed by purification over silica gel column using 5\% EtOAc in petroleum ether gave the 2-allyl-2methoxy-3,4-dihydro-2H-naphthalen-1-one 30 as colorless oil. Yield $690 \mathrm{mg}$ (65\%); [Found (ES): $\mathrm{MH}^{+}$, 217.1225, $\mathrm{C}_{14} \mathrm{H}_{17} \mathrm{O}_{2}(\mathrm{MH})$ requires $M H, 217.1223$ ]; FT IR (thin film) $1686,1601 \mathrm{~cm}^{-1}$; ${ }^{1} \mathrm{H}$ NMR (400 MHz, $\left.\mathrm{CDCl}_{3}\right) \delta 2.03-2.10\left(\mathrm{~m}, 1 \mathrm{H}, \mathrm{CH}_{2}\right), 2.38-2.44\left(\mathrm{~m}, 1 \mathrm{H}, \mathrm{CH}_{2}\right), 2.48\left(\mathrm{ddt}, J=14.56,7.59,1.05 \mathrm{~Hz}, 1 \mathrm{H}, \mathrm{CH}_{2}\right)$, $2.73\left(\mathrm{ddt}, J=14.56,6.68,1.28 \mathrm{~Hz}, 1 \mathrm{H}, \mathrm{CH}_{2}\right), 2.87\left(\mathrm{dt}, J=17.04,5.3 \mathrm{~Hz}, 1 \mathrm{H}, \mathrm{CH}_{2}\right), 3.20-3.28(\mathrm{~m}, 1 \mathrm{H}$, $\left.\mathrm{CH}_{2}\right), 3.24\left(\mathrm{~s}, 3 \mathrm{H}, \mathrm{OCH}_{3}\right), 5.13-5.19\left(\mathrm{~m}, 2 \mathrm{H}, \mathrm{CH}_{2}=\right), 5.79-5.90(\mathrm{~m}, 1 \mathrm{H}, \mathrm{CH}=), 7.24(\mathrm{~d}, J=7.67 \mathrm{~Hz}, 1 \mathrm{H}$, 
ArH), 7.32 (t, $J=7.80 \mathrm{~Hz}, 1 \mathrm{H}, \mathrm{ArH}), 7.48(\mathrm{dt}, J=7.48,1.40 \mathrm{~Hz}, 1 \mathrm{H}, \mathrm{ArH}), 8.06$ (dd, $J=7.84,1.18 \mathrm{~Hz}, 1 \mathrm{H}$, $\mathrm{ArH}) ;{ }^{13} \mathrm{C}$ NMR $\left(100 \mathrm{MHz}, \mathrm{CDCl}_{3}\right) \delta 25.39\left(\mathrm{CH}_{2}\right), 32.13\left(\mathrm{CH}_{2}\right), 36.37\left(\mathrm{CH}_{2}\right), 51.61\left(\mathrm{CH}_{3}\right), 78.65(\mathrm{C})$, $118.75\left(\mathrm{CH}_{2}\right), 126.78(\mathrm{CH}), 128.24(\mathrm{CH}), 128.80(\mathrm{CH}), 131.75(\mathrm{C}), 132.97(\mathrm{CH}), 133.59(\mathrm{CH}), 143.94(\mathrm{C})$, $196.19(\mathrm{C}) ; \mathrm{m} / z\left(\mathrm{CI}^{+}\right) 217\left(\mathrm{MH}^{+}, 30 \%\right), 187(30), 146(100)$.

\section{Nitrones 31a and 31b:}

A mixture of methoxyketone $\mathbf{3 0}$ (500 mg, $2.32 \mathrm{mmol})$, hydroxylamine hydrochloride (240 $\mathrm{mg}, 3.47 \mathrm{mmol})$, sodium hydroxide (185 mg, $4.63 \mathrm{mmol})$, absolute ethanol $(8 \mathrm{~mL})$ and water $(2 \mathrm{~mL})$ was refluxed for 3 days. Solvent was removed under reduced pressure and the residue was partitioned between EtOAc and saturated aqueous brine solution. The aqueous phase was extracted with EtOAc (10 mL x 2) and the combined organic layer was dried over anhydrous $\mathrm{Na}_{2} \mathrm{SO}_{4}$. Evaporation of the solvent followed by purification over silica gel column using neat EtOAc gave the nitrones $(\mathbf{3 1 a}+\mathbf{3 1 b})(105 \mathrm{mg}, 20 \%)$ as a mixture of diastereomers.

These were separated by hplc (DCM/ MeOH, 99:1).

Major isomer: White solid; Yield $46 \mathrm{mg}\left(9 \%\right.$ ) [Found (ES): $\mathrm{MH}^{+}, 232.1335, \mathrm{C}_{14} \mathrm{H}_{18} \mathrm{O}_{2} \mathrm{~N}(\mathrm{MH})$ requires $M H, 232.1332]$; FT IR (thin film) 2980, 2937, 2223, 1573, 1464, 1385, 1264, 1227, 1094, $1065 \mathrm{~cm}^{-1} ;{ }^{1} \mathrm{H}$ NMR (400 MHz, $\left.\mathrm{CDCl}_{3}\right) \delta 1.57\left(\mathrm{~d}, J=6.65 \mathrm{~Hz}, 3 \mathrm{H}, \mathrm{CH}_{3}\right), 1.74\left(\mathrm{dd}, J=14.02,8.90 \mathrm{~Hz}, 1 \mathrm{H}, \mathrm{CH}_{2}\right), 1.77-$ $1.85\left(\mathrm{~m}, 1 \mathrm{H}, \mathrm{CH}_{2}\right), 2.47$ (ddd, $\left.J=13.71,5.24,1.76 \mathrm{~Hz}, 1 \mathrm{H}, \mathrm{CH}_{2}\right), 2.72\left(\mathrm{dd}, J=14.0,6.96 \mathrm{~Hz}, 1 \mathrm{H}, \mathrm{CH}_{2}\right.$ ), $2.82\left(\mathrm{ddd}, J=17.0,4.0,1.48 \mathrm{~Hz}, 1 \mathrm{H}, \mathrm{CH}_{2}\right), 3.19-3.27\left(\mathrm{~m}, 1 \mathrm{H}, \mathrm{CH}_{2}\right), 3.25\left(\mathrm{~s}, 3 \mathrm{H}, \mathrm{OCH}_{3}\right), 4.46-4.52(\mathrm{~m}$, 1H, CH), 7.25 - 7.28 (m, 1H, ArH), 7.33 - 7.39 (m, 2H, ArH), 9.26 - 9.28 (m, 1H, ArH); ${ }^{13} \mathrm{C}$ NMR (100 $\left.\mathrm{MHz}, \mathrm{CDCl}_{3}\right) \delta 18.99\left(\mathrm{CH}_{3}\right), 26.67\left(\mathrm{CH}_{2}\right), 33.65\left(\mathrm{CH}_{2}\right), 37.59\left(\mathrm{CH}_{2}\right), 51.38\left(\mathrm{CH}_{3}\right), 68.73(\mathrm{CH}), 80.51(\mathrm{C})$, $125.24(\mathrm{C}), 126.65(\mathrm{CH}), 127.25(\mathrm{CH}), 128.60(\mathrm{CH}), 130.31(\mathrm{CH}), 138.26(\mathrm{C}) ; \mathrm{m} / \mathrm{z}\left(\mathrm{EI}^{+}\right) 231\left(\mathrm{M}^{+}, 20 \%\right)$, 200 (48), 128 (56), 115 (100).

Minor isomer: Colorless oil; Yield $31 \mathrm{mg}(6 \%)$; FT IR $\left(\mathrm{CH}_{2} \mathrm{Cl}_{2}\right)$ 2969, 2936, 1561, 1463, 1383, 1278, 1068 $\mathrm{cm}^{-1}$; ${ }^{1} \mathrm{H}$ NMR (400 MHz, $\left.\mathrm{CDCl}_{3}\right) \delta 1.69\left(\mathrm{~d}, J=6.9 \mathrm{~Hz}, 3 \mathrm{H}, \mathrm{CH}_{3}\right), 1.79(\mathrm{ddd}, J=13.9,13.12,5.20 \mathrm{~Hz}, 1 \mathrm{H}$, $\left.\mathrm{CH}_{2}\right), 2.13-2.24\left(\mathrm{~m}, 2 \mathrm{H}, \mathrm{CH}_{2}\right), 2.50\left(\mathrm{ddd}, \mathrm{J}=14.0,5.1,1.8 \mathrm{~Hz}, 1 \mathrm{H}, \mathrm{CH}_{2}\right), 2.77-2.83\left(\mathrm{~m}, 1 \mathrm{H}, \mathrm{CH}_{2}\right), 3.09$ - $3.18\left(\mathrm{~m}, 1 \mathrm{H}, \mathrm{CH}_{2}\right), 3.25$ (s, 3H, $\left.\mathrm{OCH}_{3}\right), 4.13-4.19$ (m, 1H, CH), 7.21 - 7.24 (m, 1H, ArH), 7.29 - 7.36 (m, 2H, ArH), 9.13 - 9.15 (m, 1H, ArH); ${ }^{13} \mathrm{C}$ NMR (100 MHz, $\left.\mathrm{CDCl}_{3}\right) \delta 19.72\left(\mathrm{CH}_{3}\right), 26.55\left(\mathrm{CH}_{2}\right), 31.05$ $\left(\mathrm{CH}_{2}\right), 33.82\left(\mathrm{CH}_{2}\right), 50.73\left(\mathrm{CH}_{3}\right), 70.25(\mathrm{CH}), 82.52(\mathrm{C}), 125.18(\mathrm{C}), 126.73(\mathrm{CH}), 127.63(\mathrm{CH}), 128.57$ $(\mathrm{CH}), 130.37(\mathrm{CH}), 138.14(\mathrm{C})$.

\section{3a-(4-Methoxyphenyl)-2-methyl-3,3a,4,5-tetrahydro-2H-benzo[g]indol-2-ol (32):}

Trifluoromethanesulfonic anhydride ( $45 \mu \mathrm{L}, 0.26 \mathrm{mmol}$ ) was added in one flush to a solution of nitrone $\mathbf{3 1}$, (major isomer) $(40 \mathrm{mg}, 0.17 \mathrm{mmol})$ in anhydrous $\mathrm{CH}_{2} \mathrm{Cl}_{2}(5 \mathrm{~mL})$ at $0{ }^{\circ} \mathrm{C}$. The resulting yellow solution was warmed to room temperature and stirred for $2.5 \mathrm{~h}$. It was diluted with $\mathrm{CH}_{2} \mathrm{Cl}_{2}(20 \mathrm{~mL})$ and washed with saturated aqueous $\mathrm{NaHCO}_{3}$ solution followed by saturated aqueous brine solution. Evaporation of the 
solvent followed by purification over silica gel column [60\% EtOAc in petroleum ether] gave $3 a-(4-$ Methoxyphenyl)-2-methyl-3,3a,4,5-tetrahydro-2H-benzo[g]indol-2-ol as a mixture of diastereomers [32a and 32b]; (25 mg, 48\%); mp 168 - $170{ }^{\circ} \mathrm{C}$; IR $\left(\mathrm{CH}_{2} \mathrm{Cl}_{2}\right)$ 3203, 2936, 1633, 1602, 1460, 1299, 1195, 1118 , $1059 \mathrm{~cm}^{-1} ;{ }^{1} \mathrm{H}$ NMR $\left(400 \mathrm{MHz}, \mathrm{CDCl}_{3}\right) \delta 1.69\left(\mathrm{~s}, 6 \mathrm{H}, \mathrm{CH}_{3}\right), 1.76-1.93\left(\mathrm{~m}, 4 \mathrm{H}, \mathrm{CH}_{2}\right), 2.50(\mathrm{dd}, J=14.6$, $\left.7.77 \mathrm{~Hz}, 2 \mathrm{H}, \mathrm{CH}_{2}\right), 2.55-2.60\left(\mathrm{~m}, 2 \mathrm{H}, \mathrm{CH}_{2}\right), 2.73-2.81\left(\mathrm{~m}, 2 \mathrm{H}, \mathrm{CH}_{2}\right), 3.08-3.24\left(\mathrm{~m}, 2 \mathrm{H}, \mathrm{CH}_{2}\right), 3.26(\mathrm{~s}$, $\left.3 \mathrm{H}, \mathrm{OCH}_{3}\right), 3.28\left(\mathrm{~s}, 3 \mathrm{H}, \mathrm{OCH}_{3}\right), 7.19-7.23(\mathrm{~m}, 2 \mathrm{H}, \mathrm{ArH}), 7.29-7.32(\mathrm{~m}, 2 \mathrm{H}, \mathrm{ArH}), 7.36-7.41(\mathrm{~m}, 2 \mathrm{H}$, ArH), $8.08(\mathrm{~d}, J=7.64 \mathrm{~Hz}, 1 \mathrm{H}, \mathrm{ArH}), 8.15(\mathrm{~d}, J=7.76 \mathrm{~Hz}, 1 \mathrm{H}, \mathrm{ArH}) ;{ }^{13} \mathrm{C} \mathrm{NMR}\left(100 \mathrm{MHz}, \mathrm{CDCl}_{3}\right) \delta 26.0$ $\left(\mathrm{CH}_{2}\right), 26.1\left(\mathrm{CH}_{2}\right), 29.5\left(\mathrm{CH}_{3}\right), 29.7\left(\mathrm{CH}_{3}\right), 31.3\left(\mathrm{CH}_{2}\right), 33.0\left(\mathrm{CH}_{2}\right), 44.9\left(\mathrm{CH}_{2}\right), 45.3\left(\mathrm{CH}_{2}\right), 51.4\left(\mathrm{CH}_{3}\right)$, $52.3\left(\mathrm{CH}_{3}\right), 86.8(\mathrm{C}), 86.9(\mathrm{C}), 99.1(\mathrm{C}), 100.9(\mathrm{C}), 126.9(\mathrm{CH}), 127.3(\mathrm{CH}), 127.6(\mathrm{CH}), 128.4(\mathrm{C}), 128.7$ (C), $129.0(\mathrm{CH}), 131.5(\mathrm{CH}), 131.6(\mathrm{CH}), 141.3(\mathrm{C}), 141.3(\mathrm{C}), 169.5(\mathrm{C}), 171.7(\mathrm{C}) . \mathrm{m} / z\left(\mathrm{CI}^{+}\right) 231\left(\mathrm{M}^{+}\right.$, $22 \%), 184(100 \%)$.

Fractional crystallization led to one isomer, which was subjected to X-ray crystal structure determination.
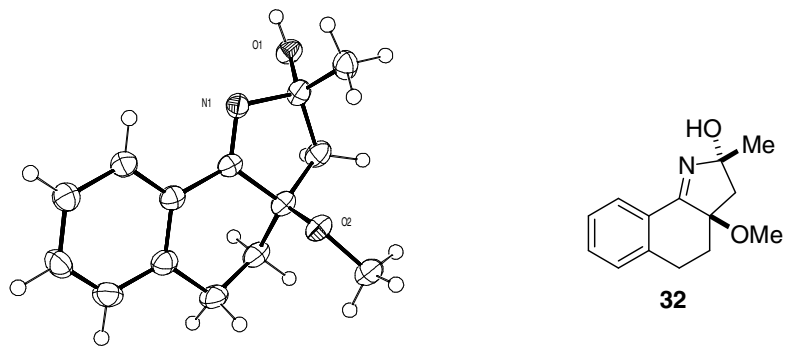

32

Figure. ORTEP view of one of the two crystallographically independent molecules of compound 32, thermal ellipsoids are drawn at the $50 \%$ probability level.

Crystal data for 32: Measurements were made with a Nonius Kappa CCD diffractometer on a colorless crystal cut from a plate, sample size $0.20 \times 0.18 \times 0.04 \mathrm{~mm}$. Found $\mathrm{C}_{14} \mathrm{H}_{17} \mathrm{NO}_{2}, M_{r}=231.29$, orthorhombic, space group Pbca, a = 17.4562(7), b= 15.5902(4), c=17.8949(7) $\AA, V=4870.0(3) \AA^{3}, Z=16, \rho_{\text {calc }}=1.262 \mathrm{~g}$ $\mathrm{cm}^{-3}, \mathrm{Mo}_{\mathrm{K} \alpha}$ radiation, $\lambda=0.71073 \AA, \mu=0.084 \mathrm{~mm}^{-1}, T=123 \mathrm{~K} ; 43642$ reflections were collected, 4315 were unique, $R_{\text {int }} 0.103$; final refinement to convergence on $F^{2}$ with all non-H atoms anisotropic gave $R=$ $0.0592\left(F, 2484\right.$ obs. data only) and $R_{\mathrm{w}}=0.1427\left(F^{2}\right.$, all data), GOF $=1.018,319$ refined parameters. $\mathrm{H}$ atoms of $\mathrm{OH}$ groups were refined isotropically but all other $\mathrm{H}$ atoms were placed in calculated positions and with riding modes. Residual electron density max. and min. 0.451 and $-0.228 \mathrm{e} \AA^{-3}$.

\section{Spiro[4.5]dec-6-ene (36): ${ }^{4}$}

Dry diglyme (175 mL), sodium methoxide (14.9 g, $275 \mathrm{mmol})$ and $N^{\prime}$-[(6E)-spiro[4.5]dec-6ylidene]benzenesulfonohydrazide $(38.0 \mathrm{~g}, 125 \mathrm{mmol})$ were placed in a 3-necked flask equipped with a magnetic stirrer and a condenser. The reaction was refluxed for $1.5 \mathrm{~h}$ before being cooled to rt. Water (100 $\mathrm{mL})$ was added to the reaction mixture. An azeotropic distillation $\left(85-99{ }^{\circ} \mathrm{C}\right.$ at $\left.760 \mathrm{mmHg}\right)$ of the reaction mixture gave spiro[4.5]dec-6-ene 36 as a clear oil. Yield $13.3 \mathrm{~g}$ (81\%); FT IR (thin film) 3012, 2930, 2857, 
1444, 940, $731 \mathrm{~cm}^{-1} ;{ }^{1} \mathrm{H}$ NMR (400 MHz, $\left.\mathrm{CDCl}_{3}\right) \delta 1.41-1.57\left(\mathrm{~m}, 6 \mathrm{H}, \mathrm{CH}_{2}\right), 1.58$ - $1.69\left(\mathrm{~m}, 6 \mathrm{H}, \mathrm{CH}_{2}\right)$, $1.94-1.98\left(\mathrm{~m}, 2 \mathrm{H}, \mathrm{CH}_{2}\right), 5.47-5.50(\mathrm{~m}, 1 \mathrm{H},=\mathrm{CH}), 5.56-5.60(\mathrm{~m}, 1 \mathrm{H},=\mathrm{CH}) ;{ }^{13} \mathrm{C} \mathrm{NMR}\left(100 \mathrm{MHz}, \mathrm{CDCl}_{3}\right)$ $\delta 21.2\left(\mathrm{CH}_{2}\right), 24.8\left(\mathrm{CH}_{2}\right), 24.8\left(\mathrm{CH}_{2}\right), 25.9\left(\mathrm{CH}_{2}\right), 35.6\left(\mathrm{CH}_{2}\right), 40.8\left(\mathrm{CH}_{2}\right), 40.8\left(\mathrm{CH}_{2}\right), 41.0(\mathrm{C}), 125.2(\mathrm{CH})$, $137.3(\mathrm{CH}) ; \mathrm{m} / z\left(\mathrm{EI}^{+}\right) 136\left(\mathrm{M}^{+}, 42 \%\right) 121$ (26), 107 (52), 94, (60), 79 (100), 67 (35).

\section{4-Methoxyphenyllithium:}

Diethyl ether $(70 \mathrm{~mL})$ and 4-iodoanisole $(15.7 \mathrm{~mL}), 125 \mathrm{mmol})$ were added to a flame-dried flask under nitrogen. The temperature was lowered to $0{ }^{\circ} \mathrm{C}$ before the dropwise addition of $2.5 \mathrm{M} \mathrm{BuLi}(49.8 \mathrm{~mL}, 125$ $\mathrm{mmol})$. The reaction was then stirred at $\mathrm{rt}$ for $1 \mathrm{~h}$. The 4-methoxyphenyllithium thus prepared was used directly in the next reaction without further purification.

\section{7-Oxaspiro[bicyclo[4.1.0]heptane-2,1'-cyclopentane $(37):^{5}$}

Diethyl ether $(100 \mathrm{~mL})$ and sodium hydrogen carbonate $(5.9 \mathrm{~g}, 71.3 \mathrm{mmol})$ were added to a 3-necked flask, followed by spiroalkene $36(9.7 \mathrm{~g}, 75 \mathrm{mmol})$ and $m \mathrm{CPBA}(77 \%, 15.9 \mathrm{~g}, 71.3 \mathrm{mmol})$. The reaction was stirred for $10 \mathrm{~min}$ and then the reaction mixture was diluted with water $(100 \mathrm{~mL})$ and extracted with diethyl ether $(2 \times 100 \mathrm{~mL})$. The combined organic extracts were washed with brine before drying over anhydrous magnesium sulfate, filtering and concentrating under reduced pressure. Column chromatography [petroleum ether/ethyl acetate (10:1)] gave 7-oxaspiro[bicyclo[4.1.0]heptane-2,1'-cyclopentane 37 as a colorless oil (10.5 g, 97\%); [Found (ES): $\mathrm{MH}^{+}, 153.1280, \mathrm{C}_{10} \mathrm{H}_{17} \mathrm{O}(\mathrm{MH})$ requires $M H, 153.1279$ ]; IR (thin film) 2939, 2863, 1453, $1215 \mathrm{~cm}^{-1} ;{ }^{1} \mathrm{H}$ NMR (400 MHz, $\left.\mathrm{CDCl}_{3}\right) \delta 1.03\left(\mathrm{~m}, 1 \mathrm{H}, \mathrm{CH}_{2}\right), 1.07-1.37(\mathrm{~m}, 6 \mathrm{H}$, $\left.\mathrm{CH}_{2}\right), 1.38-1.63\left(\mathrm{~m}, 4 \mathrm{H}, \mathrm{CH}_{2}\right), 1.71-1.85\left(\mathrm{~m}, 3 \mathrm{H}, \mathrm{CH}_{2}\right), 2.83(\mathrm{~d}, 1 \mathrm{H}, \mathrm{J}=3.9 \mathrm{~Hz}, \mathrm{OCH}), 3.20(\mathrm{dt}, 1 \mathrm{H}, \mathrm{J}=$ 3.9, $1.0 \mathrm{~Hz}, \mathrm{OCH}) ;{ }^{13} \mathrm{C} \mathrm{NMR}\left(100 \mathrm{MHz}, \mathrm{CDCl}_{3}\right) \delta 17.9\left(\mathrm{CH}_{2}\right), 24.1\left(\mathrm{CH}_{2}\right), 24.6\left(\mathrm{CH}_{2}\right), 25.0\left(\mathrm{CH}_{2}\right), 32.9$ $\left(\mathrm{CH}_{2}\right), 36.5\left(\mathrm{CH}_{2}\right), 37.7\left(\mathrm{CH}_{2}\right), 42.0(\mathrm{C}), 53.9(\mathrm{CH}), 60.2(\mathrm{CH}) ; \mathrm{m} / z\left(\mathrm{CI}^{+}\right) 170\left(\mathrm{MNH}_{4}^{+}, 24 \%\right) 153(22), 134$ (100), 107 (32).

\section{7-(4-Methoxyphenyl)spiro[4.5]decan-6-ol (38):}

Diethyl ether $(75 \mathrm{~mL})$ and copper (I) cyanide $(3.52 \mathrm{~g}, 39.0 \mathrm{mmol})$ cooled to $-78^{\circ} \mathrm{C}$ at which point $1.3 \mathrm{M}$ (4methoxyphenyl)lithium (57.5 mL $75.6 \mathrm{mmol})$ was added. The reaction was allowed to stir for $10 \mathrm{~min}$ before the addition of epoxide $37(5.4 \mathrm{~g}, 35.7 \mathrm{mmol})^{5}$, followed by $\mathrm{BF}_{3} \cdot \mathrm{OEt}_{2}(7.38 \mathrm{~mL}, 39.0 \mathrm{mmol})$. The temperature of the reaction was then allowed to warm to $\mathrm{rt}$ and stir overnight. The reaction was quenched by the careful addition of saturated ammonium chloride solution $(50 \mathrm{~mL})$ and extracted with ethyl acetate $(2$ X $50 \mathrm{~mL}$ ). The combined organic extracts were washed with brine before drying over anhydrous magnesium sulfate, filtering and concentrating under reduced pressure. Column chromatography [petroleum ether/ethyl acetate (95:5 v/v)] gave 7-(4-methoxyphenyl)spiro[4.5]decan-6-ol 38 as an orange solid (4.5 g, 49\%); mp 56-58 ${ }^{\circ} \mathrm{C}$; [Found (ES): $\mathrm{MNH}_{4}{ }^{+}, 278.2121 \mathrm{C}_{17} \mathrm{H}_{28} \mathrm{NO}_{2}\left(\mathrm{MNH}_{4}\right)$ requires $M N H_{4}$, 278.2120]; FT IR (KBr disc) 3354, 2924, 2857, 1607, 1508, 1440, 1242, 1176, 1024, $810 \mathrm{~cm}^{-1}$; ${ }^{1} \mathrm{H}$ NMR $\left(400 \mathrm{MHz}, \mathrm{CDCl}_{3}\right) \delta 1.33-1.91\left(\mathrm{~m}, 13 \mathrm{H}, \mathrm{CH}_{2}\right), 2.03-2.06\left(\mathrm{~m}, 1 \mathrm{H}, \mathrm{CH}_{2}\right), 2.51-2.58(\mathrm{~m}, 1 \mathrm{H}, \mathrm{CH}), 3.58-$ 
$3.61(\mathrm{~m}, 1 \mathrm{H}, \mathrm{CH}), 3.87\left(\mathrm{~s}, 3 \mathrm{H}, \mathrm{OCH}_{3}\right), 6.96(\mathrm{~d}, \mathrm{~J}=8.6 \mathrm{~Hz}, 2 \mathrm{H}, \mathrm{ArH}), 7.26(\mathrm{~d}, \mathrm{~J}=8.6 \mathrm{~Hz}, 2 \mathrm{H}, \operatorname{ArH}) ;{ }^{13} \mathrm{C}$ NMR (100 MHz, $\left.\mathrm{CDCl}_{3}\right) \delta 23.3\left(\mathrm{CH}_{2}\right), 26.0\left(\mathrm{CH}_{2}\right), 27.1\left(\mathrm{CH}_{2}\right), 29.1\left(\mathrm{CH}_{2}\right), 34.2\left(\mathrm{CH}_{2}\right), 39.0\left(\mathrm{CH}_{2}\right), 39.2$ $\left(\mathrm{CH}_{2}\right), 48.2(\mathrm{C}), 49.2(\mathrm{CH}), 55.7\left(\mathrm{CH}_{3}\right), 80.0(\mathrm{CH}), 114.6(\mathrm{CH}), 129.3(\mathrm{CH}), 135.8(\mathrm{C}), 158.9(\mathrm{C}) ; \mathrm{m} / z\left(\mathrm{EI}^{+}\right)$ $260\left(\mathrm{M}^{+}, 39 \%\right) 214$ (16), 199, (14), 147 (31), 134 (34), 121 (100), 91 (31), 77 (22), 67 (26), 55 (18).

\section{7-(4-Methoxyphenyl)spiro[4.5]decan-6-one (39)}

Freshly distilled benzene $(100 \mathrm{~mL})$ and alcohol $38(2.5 \mathrm{~g}, 9.6 \mathrm{mmol})$ were added to a flame-dried flask under nitrogen containing PDC (10.9 g, $28.8 \mathrm{mmol})$. The reaction was refluxed overnight before cooling to rt, at which point water $(50 \mathrm{~mL})$ was added. The reaction was then filtered and washed with ethyl acetate. The organic phase was separated and the aqueous phase backwashed with ethyl acetate ( 2 X $100 \mathrm{~mL})$. The combined organic extracts were then washed with brine solution before drying over anhydrous magnesium sulfate, filtering and concentrating under reduced pressure. Column chromatography [petroleum ether/ethyl acetate $(95: 5 \mathrm{v} / \mathrm{v})$ ] gave 7-(4-methoxyphenyl)spiro [4.5] decan-6-one 39 as a viscous yellow oil (1.93 g, 61\%); [Found (ES): $\mathrm{MH}^{+}, 259.1696, \mathrm{C}_{17} \mathrm{H}_{23} \mathrm{O}(\mathrm{MH})$ requires $M H, 259.1698$ ]; FT IR (thin film) 2938, 2863, 1706, 1613, 1513, 1449, 1292, 1247, 1038, $828 \mathrm{~cm}^{-1}$; ${ }^{1} \mathrm{H}$ NMR (400 MHz, $\left.\mathrm{CDCl}_{3}\right) \delta 1.09-1.16(\mathrm{~m}, 1 \mathrm{H}$, $\left.\mathrm{CH}_{2}\right), 1.58-1.98\left(\mathrm{~m}, 11 \mathrm{H}, \mathrm{CH}_{2}\right), 2.19-2.22\left(\mathrm{~m}, 1 \mathrm{H}, \mathrm{CH}_{2}\right), 2.47-2.51\left(\mathrm{~m}, 1 \mathrm{H}, \mathrm{CH}_{2}\right), 3.75-3.80(\mathrm{~m}, 1 \mathrm{H}$, $\mathrm{CH}), 3.82\left(\mathrm{~s}, 3 \mathrm{H}, \mathrm{OCH}_{3}\right), 6.89(\mathrm{~d}, J=8.6 \mathrm{~Hz}, 2 \mathrm{H}, \mathrm{ArH}), 7.07(\mathrm{~d}, J=8.6 \mathrm{~Hz}, 2 \mathrm{H}, \mathrm{ArH}) ;{ }^{13} \mathrm{C} \mathrm{NMR}(100 \mathrm{MHz}$, $\left.\mathrm{CDCl}_{3}\right) \delta 23.2\left(\mathrm{CH}_{2}\right), 24.9\left(\mathrm{CH}_{2}\right), 25.6\left(\mathrm{CH}_{2}\right), 34.7\left(\mathrm{CH}_{2}\right), 35.7\left(\mathrm{CH}_{2}\right), 36.8\left(\mathrm{CH}_{2}\right), 40.5\left(\mathrm{CH}_{2}\right), 53.7(\mathrm{CH})$, $55.3\left(\mathrm{CH}_{3}\right), 113.8(\mathrm{CH}), 129.7(\mathrm{CH}), 131.5(\mathrm{C}), 158.4(\mathrm{C}), 212.9(\mathrm{C}): \mathrm{m} / z\left(\mathrm{EI}^{+}\right) 259\left(\mathrm{MH}^{+}, 8 \%\right), 258(31)$, 230 (20), 160 (26), 147 (83), 134 (100), 121 (67), 91 (48), 67 (41), 41 (51).

\section{7-Allyl-7-(4-methoxyphenyl)spiro[4.5]decan-6-one (40):}

Potassium tert-butoxide $(2.19 \mathrm{~g}, 19.7 \mathrm{mmol})$ was stirred in toluene $(90 \mathrm{~mL})$ at room temperature. This was followed by the addition of ketone 39 (2.5 g, $9.9 \mathrm{mmol})$. Allyl bromide (4.77 g, $39.4 \mathrm{mmol})$ was then added and the mixture was heated at reflux for $24 \mathrm{~h}$. The reaction was then cooled and water $(30 \mathrm{~mL})$ added, followed by ethyl acetate $(100 \mathrm{~mL})$. The organic phase was dried over anhydrous magnesium sulfate, filtered and concentrating under reduced pressure. Column chromatography [petroleum ether/ethyl acetate (95:5 v/v)] gave 7-allyl-7-(4-methoxyphenyl)spiro[4.5] decan-6-one 40 as a colorless oil (2.65 g, 89\%); [Found (ES): $\mathrm{MNH}_{4}{ }^{+}, 316.2277, \mathrm{C}_{20} \mathrm{H}_{30} \mathrm{NO}_{2}\left(\mathrm{MNH}_{4}\right)$ requires $M \mathrm{MH}_{4}$ 316.2276]; FT IR (thin film) 3071, 2938, 2868, 1691, 1608, 1511, 1463, 1251, 914, $831 \mathrm{~cm}^{-1} ;{ }^{1} \mathrm{H}$ NMR (400 MHz, $\left.\mathrm{CDCl}_{3}\right) \delta 1.12-1.17(\mathrm{~m}, 1 \mathrm{H}$, $\left.\mathrm{CH}_{2}\right), 1.21-1.30\left(\mathrm{~m}, 2 \mathrm{H}, \mathrm{CH}_{2}\right), 1.46-1.51\left(\mathrm{~m}, 2 \mathrm{H}, \mathrm{CH}_{2}\right), 1.59-1.84\left(\mathrm{~m}, 6 \mathrm{H}, \mathrm{CH}_{2}\right), 1.97-2.03(\mathrm{~m}, 1 \mathrm{H}$, $\left.\mathrm{CH}_{2}\right), 2.34-2.40\left(\mathrm{~m}, 1 \mathrm{H}, \mathrm{CH}_{2}\right), 2.47\left(\mathrm{dd}, 1 \mathrm{H}, \mathrm{J}=13.9,7.6 \mathrm{~Hz}, \mathrm{CH}_{2}\right), 2.59\left(\mathrm{~m}, 1 \mathrm{H}, \mathrm{CH}_{2}\right), 2.62(\mathrm{dd}, 1 \mathrm{H}, \mathrm{J}=$ 13.9, $\left.6.8 \mathrm{~Hz}, \mathrm{CH}_{2}\right), 3.87\left(\mathrm{~s}, 3 \mathrm{H}, \mathrm{OCH}_{3}\right), 4.94-4.99\left(\mathrm{~m}, 2 \mathrm{H},=\mathrm{CH}_{2}\right), 5.39-5.49(\mathrm{~m}, 1 \mathrm{H},=\mathrm{CH}), 6.91(\mathrm{dd}, 2 \mathrm{H}$, $J=8.6,2.1 \mathrm{~Hz}, \mathrm{ArH}), 7.25(\mathrm{dd}, 2 \mathrm{H}, J=8.6,2.1 \mathrm{~Hz}, \mathrm{ArH}) ;{ }^{13} \mathrm{C} \mathrm{NMR}\left(100 \mathrm{MHz} \mathrm{CDCl}_{3}\right) \delta 18.9\left(\mathrm{CH}_{2}\right), 24.3$ $\left(\mathrm{CH}_{2}\right), 25.0\left(\mathrm{CH}_{2}\right), 32.6\left(\mathrm{CH}_{2}\right), 36.4\left(\mathrm{CH}_{2}\right), 37.7\left(\mathrm{CH}_{2}\right), 38.1\left(\mathrm{CH}_{2}\right), 46.9\left(\mathrm{CH}_{2}\right), 54.5(\mathrm{C}), 55.3\left(\mathrm{CH}_{3}\right), 57.1$ (C), $113.9(\mathrm{CH}), 117.5\left(\mathrm{CH}_{2}\right), 127.7(\mathrm{CH}), 132.6(\mathrm{C}), 135.0(\mathrm{CH}), 158.3(\mathrm{C}), 215.5(\mathrm{C}) ; \mathrm{m} / z\left(\mathrm{CI}^{+}\right) 316$ $\left(\mathrm{MNH}_{4}^{+}, 100 \%\right) 299$ (37), 276 (7), 257 (10), 229 (8), 147 (4), 121 (5). 


\section{[7-(4-Methoxyphenyl)-6-oxospiro[4.5]dec-7-yl]acetaldehyde (41):}

Allyl ketone $40(2.54 \mathrm{~g}, 9.87 \mathrm{mmol})$ was stirred in dichloromethane $(60 \mathrm{~mL})$ at $-78^{\circ} \mathrm{C}$ at which point ozone was pumped through the reaction mixture. This was continued for $1 \mathrm{~h}$ after the reaction solution had turned blue. The ozone was then replaced with oxygen and pumped through until the reaction solution became colorless. Dimethyl sulfide $(6.5 \mathrm{~mL}, 87.5 \mathrm{mmol})$ was then added to the reaction and allowed to warm to $\mathrm{rt}$ and stir overnight. It was diluted with water and extracted with $\mathrm{CH}_{2} \mathrm{Cl}_{2}(2 \times 100 \mathrm{~mL})$. The combined organic extracts were dried over anhydrous sodium sulfate, filtered and concentrated. Column chromatography [petroleum ether/ethyl acetate $(90: 10 \mathrm{v} / \mathrm{v})$ ] gave the compound $\mathbf{4 1}$ as a colorless oil (2.1g, 80\%); [Found (ES) $\mathrm{MH}^{+}, 301.1796, \mathrm{C}_{19} \mathrm{H}_{25} \mathrm{O}_{3}(\mathrm{MH})$ requires $M H, 301.1798$ ]; FT IR (thin film) 2935, 2869, 2733, 1720, 1693, 1607, 1511, 1464, 1253, 1185, 1034, $831 \mathrm{~cm}^{-1} ;{ }^{1} \mathrm{H}$ NMR (400 MHz, $\left.\mathrm{CDCl}_{3}\right) \delta 1.14(\mathrm{~m}$, 1H, $\left.\mathrm{CH}_{2}\right), 1.24\left(\mathrm{~m}, 2 \mathrm{H}, \mathrm{CH}_{2}\right), 1.46-1.79\left(\mathrm{~m}, 7 \mathrm{H}, \mathrm{CH}_{2}\right), 1.94-2.06\left(\mathrm{~m}, 2 \mathrm{H}, \mathrm{CH}_{2}\right), 2.32\left(\mathrm{~m}, 1 \mathrm{H}, \mathrm{CH}_{2}\right), 2.65$ $\left(\mathrm{d}, 1 \mathrm{H}, \mathrm{J}=14.0 \mathrm{~Hz}, \mathrm{CH}_{2}\right), 2.72\left(\mathrm{dd}, 1 \mathrm{H}, \mathrm{J}=16.2,2.1 \mathrm{~Hz}, \mathrm{CH}_{2}\right), 2.86\left(\mathrm{dd}, 1 \mathrm{H}, \mathrm{J}=16.2,2.1 \mathrm{~Hz}, \mathrm{CH}_{2}\right), 3.85$ (s, $\left.3 \mathrm{H}, \mathrm{OCH}_{3}\right), 6.91(\mathrm{dd}, \mathrm{J}=8.8,2.0 \mathrm{~Hz}, 2 \mathrm{H}, \mathrm{ArH}), 7.26(\mathrm{dd}, \mathrm{J}=8.8,2.0 \mathrm{~Hz}, 2 \mathrm{H}, \mathrm{ArH}), 9.53(\mathrm{t}, \mathrm{J}=2.1 \mathrm{~Hz}, 1 \mathrm{H}$, $\mathrm{CHO}) ;{ }^{13} \mathrm{C}$ NMR $\left(100 \mathrm{MHz}, \mathrm{CDCl}_{3}\right) \delta 18.5\left(\mathrm{CH}_{2}\right), 24.0\left(\mathrm{CH}_{2}\right), 24.7\left(\mathrm{CH}_{2}\right), 32.8\left(\mathrm{CH}_{2}\right), 36.4\left(\mathrm{CH}_{2}\right), 36.7$ $\left(\mathrm{CH}_{2}\right), 37.8\left(\mathrm{CH}_{2}\right), 53.2(\mathrm{C}), 54.9\left(\mathrm{CH}_{3}\right), 55.2\left(\mathrm{CH}_{2}\right), 56.5(\mathrm{C}), 114.0(\mathrm{CH}), 127.1(\mathrm{CH}), 130.9(\mathrm{C}), 158.5(\mathrm{C})$, $201.1(\mathrm{CH}), 214.1(\mathrm{C}), \mathrm{m} / z\left(\mathrm{EI}^{+}\right) 300\left(\mathrm{MH}^{+}, 20 \%\right), 258$ (31), 202 (33), 189 (45), 148 (100), 121 (86), 91 (47), 67 (66).

\section{3a'-(4-Methoxyphenyl)-2',3',3a',4',5',6'-hexahydrospiro[cyclopentane-1,7' indole]1'-oxide (43).}

Ethanol $(50 \mathrm{~mL})$, water $(10 \mathrm{~mL})$, sodium hydroxide $(540 \mathrm{mg}, 13.52 \mathrm{mmol})$, hydroxylamine hydrochloride (180 mg, $3.25 \mathrm{mmol})$ and aldehyde 41 (1.5 g, $5.2 \mathrm{mmol})$ were added to a 3-necked flask and refluxed for 18 h. The reaction mixture was concentrated under reduced pressure before partitioning between brine (100 $\mathrm{mL})$ and ethyl acetate $(100 \mathrm{~mL})$. The aqueous layer was backwashed with ethyl acetate $(2 \mathrm{X} 50 \mathrm{~mL})$. The organic extracts were then combined before drying over anhydrous magnesium sulfate, filtering and concentrating under reduced pressure. Column chromatography [petroleum ether/ethyl acetate $(75: 25 \mathrm{v} / \mathrm{v})$ ] gave [7-(4-methoxyphenyl)-6-oxospiro [4.5]dec-7-yl]acetaldehyde oxime $\mathbf{4 2}$ as a foam (1.38 g,), which was used without further purification.

Ethanol (30 mL) and aldoxime $42(1.37 \mathrm{~g}, 4.52 \mathrm{mmol})$ were added to a 3-necked flask under nitrogen. This was followed by the addition of methanolic hydrochloric acid $(40 \mathrm{~mL})$. Sodium cyanoborohydride $(0.85 \mathrm{~g}$, $13.56 \mathrm{mmol}$ ) was added to the mixture portion wise along with hydrochloric acid solution $(6 \mathrm{~N})$ to maintain an acidity of $p \mathrm{H} 3$. The reaction was then stirred overnight before neutralizing with potassium carbonate (5 g). The solvent was removed under reduced pressure and the residue subjected to column chromatography $\left[\mathrm{CH}_{2} \mathrm{Cl}_{2} /\right.$ methanol $\left.(90: 10 \mathrm{v} / \mathrm{v})\right]$, this gave $3 a^{\prime}-(4-$ methoxyphenyl)-2',3',3a',4',5',6'-hexahydrospiro[cyclopentane-1,7' indole] 1'-oxide $\mathbf{4 3}$ as a viscous colorless oil (348 mg, $19 \%$ from aldehyde 41); [Found (ES): $\mathrm{M}^{+}, 299.1885, \mathrm{C}_{19}, \mathrm{H}_{25} \mathrm{NO}_{2}$ (M) requires $\left.M, 299.1885\right]$; IR (thin film) 2944, 2867, 1607, 1579, 1512, 1463 , 1362, 1285, 1251, 1183, 1101, 1032, $831 \mathrm{~cm}^{-1}$; ${ }^{1} \mathrm{H}$ NMR (400 MHz, $\left.\mathrm{CDCl}_{3}\right) \delta 1.11-1.69\left(\mathrm{~m}, 9 \mathrm{H}, \mathrm{CH}_{2}\right)$, $1.87-1.98\left(\mathrm{~m}, 3 \mathrm{H}, \mathrm{CH}_{2}\right), 2.04-2.17\left(\mathrm{~m}, 1 \mathrm{H}, \mathrm{CH}_{2}\right), 2.64-2.70\left(\mathrm{~m}, 3 \mathrm{H}, \mathrm{CH}_{2}\right), 3.75-3.86\left(\mathrm{~m}, 4 \mathrm{H}, \mathrm{CH}_{3}\right.$, 
$\left.\mathrm{CH}_{2}\right), 4.09-4.17\left(\mathrm{~m}, 1 \mathrm{H}, \mathrm{CH}_{2}\right), 6.87(\mathrm{~d}, J=8.6 \mathrm{~Hz}, 2 \mathrm{H}, \mathrm{ArH}), 7.09$ (d, $\left.J=8.6 \mathrm{~Hz}, 2 \mathrm{H}, \mathrm{ArH}\right) ;{ }^{13} \mathrm{C} \mathrm{NMR}$ $\left(100 \mathrm{MHz}, \mathrm{CDCl}_{3}\right) \delta 17.7\left(\mathrm{CH}_{2}\right), 25.7\left(\mathrm{CH}_{2}\right), 25.8\left(\mathrm{CH}_{2}\right), 33.1\left(\mathrm{CH}_{2}\right), 33.2\left(\mathrm{CH}_{2}\right), 34.3\left(\mathrm{CH}_{2}\right), 35.5\left(\mathrm{CH}_{2}\right)$, $37.7\left(\mathrm{CH}_{2}\right), 45.0(\mathrm{C}), 53.1(\mathrm{C}), 55.4\left(\mathrm{CH}_{3}\right), 61.4\left(\mathrm{CH}_{2}\right), 114.6(\mathrm{CH}), 126.6(\mathrm{CH}), 135.8(\mathrm{C}), 155.8(\mathrm{C}), 158.6$ (C); m/z (EI+) $299\left(\mathrm{M}^{+}, 49 \%\right) 258$ (99), 242 (100), 167 (12), 149 (26), 121 (19).

\section{0-(4-Methoxyphenyl)-7-azaspiro-[4,8]-tridec-10-en-6-one (44):}

Freshly distilled $\mathrm{CH}_{2} \mathrm{Cl}_{2}(20 \mathrm{~mL})$ was added to a flame-dried flask under nitrogen. This was followed by the addition of nitrone $22(500 \mathrm{mg}, 1.6 \mathrm{mmol})$ dissolved in anhydrous $\mathrm{CH}_{2} \mathrm{Cl}_{2}(5 \mathrm{~mL})$. Trifluoromethanesulfonic anhydride $(410 \mu \mathrm{L}, 2.5 \mathrm{mmol})$ was then added to the reaction mixture in a single flush. The reaction was stirred for $2 \mathrm{~h}$ before the addition of water. $\mathrm{CH}_{2} \mathrm{Cl}_{2}(3 \times 20 \mathrm{~mL})$ was used to extract the reaction. The combined organic extracts were washed with brine before drying over anhydrous sodium sulfate, filtering and concentrating under reduced pressure. Column chromatography [DCM/methanol (95:5 $\mathrm{v} / \mathrm{v})]$, followed by HPLC $\left[\mathrm{CH}_{2} \mathrm{Cl}_{2} /\right.$ methanol 95:5 v/v)] led to the isolation of 10-(4-methoxyphenyl)-7azaspiro-[4, 8]-tridec-10-en-6-one 44 as a brown oil (163 mg, 35\%) as a pair of diastereomers; [Found (ES): $\mathrm{M}^{+}, 299.1888, \mathrm{C}_{19} \mathrm{H}_{25} \mathrm{NO}_{2}(\mathrm{M})$ requires $M$, 299.1885]; IR (thin film) 3226, 2942, 2837, 1693, 1609, 1580 , 1511, 1461, 1440, 1276, 1250, 1183, 1033, $828 \mathrm{~cm}^{-1} ;{ }^{1} \mathrm{H}$ NMR (400 MHz, $\left.\mathrm{CDCl}_{3}\right) \delta 1.34-1.37(\mathrm{~m}, 1 \mathrm{H}$, $\left.\mathrm{CH}_{2}\right), 1.54$ - $2.06\left(\mathrm{~m}, 8 \mathrm{H}, \mathrm{CH}_{2}\right), 2.12$ - $2.17\left(\mathrm{~m}, 2 \mathrm{H}, \mathrm{CH}_{2}\right), 2.4$ - $2.29\left(\mathrm{~m}, 2 \mathrm{H}, \mathrm{CH}_{2}\right), 2.53$ - $2.56\left(\mathrm{~m}, 1 \mathrm{H}, \mathrm{CH}_{2}\right)$, $3.24-3.35\left(\mathrm{~m}, 2 \mathrm{H}, \mathrm{CH}_{2}\right), 3.79\left(\mathrm{~s}, 3 \mathrm{H}, \mathrm{CH}_{3}\right), 5.15-5.20$ and $5.25-5.27(2 \mathrm{x} \mathrm{m}, 1 \mathrm{H},=\mathrm{CH}), 5.30$ and $6.15(2$ $\mathrm{x}$ brs, $1 \mathrm{H}, \mathrm{NH}), 6.85-6.88(\mathrm{~m}, 2 \mathrm{H}, \mathrm{ArH}), 7.39-7.42(\mathrm{~m}, 2 \mathrm{H}, \mathrm{ArH}) ;{ }^{13} \mathrm{C} \mathrm{NMR}\left(100 \mathrm{MHz}, \mathrm{CDCl}_{3}\right) \delta 23.0$ $\left(\mathrm{CH}_{2}\right), 23.6\left(\mathrm{CH}_{2}\right), 25.1\left(\mathrm{CH}_{2}\right), 26.5\left(\mathrm{CH}_{2}\right), 26.6\left(\mathrm{CH}_{2}\right), 28.7\left(\mathrm{CH}_{2}\right), 31.5\left(\mathrm{CH}_{2}\right), 32.5\left(\mathrm{CH}_{2}\right), 33.7\left(\mathrm{CH}_{2}\right)$, $33.8\left(\mathrm{CH}_{2}\right), 33.9\left(\mathrm{CH}_{2}\right), 35.1\left(\mathrm{CH}_{2}\right), 38.8\left(\mathrm{CH}_{2}\right), 38.8\left(\mathrm{CH}_{2}\right), 39.2\left(\mathrm{CH}_{2}\right), 39.2\left(\mathrm{CH}_{2}\right), 50.4(\mathrm{C}), 55.4\left(\mathrm{CH}_{3}\right)$, $113.9(\mathrm{CH}), 119.5(\mathrm{CH}), 123.8(\mathrm{CH}), 127.8(\mathrm{CH}), 127.8(\mathrm{CH}), 133.5(\mathrm{C}), 133.5(\mathrm{C}), 143.8(\mathrm{C}), 144.4(\mathrm{C})$, 158.5 (C), 180.5 (C), 180.6 (C); m/z (EI $) 299$ (M+, $45 \%) 249$ (10), 204 (25), 191 (100), 133 (46), 121 (20).

The structure of the amide was further confirmed by ${ }^{1} \mathrm{H},{ }^{1} \mathrm{H}$ 2D COSY, ${ }^{1} \mathrm{H},{ }^{13} \mathrm{C} 2 \mathrm{D}$ HSQC and ${ }^{1} \mathrm{H},{ }^{13} \mathrm{C}$ 2D HMBC experiments.

\section{Fragmentative Rearrangement of Nitrone 46:}

A two necked $25 \mathrm{ml}$ round-bottomed flask fitted with a rubber septum and a magnetic stirbar was flamedried under vacuum, backfilled with dry argon, charged with a solution of the nitrone 46 (0.194 g, 0.5599 mmol, $1.0 \mathrm{eq})$ in dry, freshly distilled and degassed dichloromethane (10 ml, degassed with argon). The solution was purged with a stream of argon gas and was cooled to $0{ }^{\circ} \mathrm{C}$. Trifluoromethanesulfonic anhydride ( $0.38 \mathrm{ml}, 2.24 \mathrm{mmol}, 4.0 \mathrm{eq}$, handled inside a glove box under a nitrogen atmosphere) was added to the solution at $0{ }^{\circ} \mathrm{C}$ under an argon atmosphere. The reaction mixture immediately turned red which later decolorized to pale-yellow after $5 \mathrm{~min}$. The reaction mixture was allowed to stir at $0{ }^{\circ} \mathrm{C}$ for $1 \mathrm{~h}$, then warmed to r.t. for $1.5 \mathrm{~h}$, and continued to stir at r.t. for an additional $9.5 \mathrm{~h}$ (total time $=12 \mathrm{~h}$ ) under an argon atmosphere. A yellowish brown semi-solid was observed during the course of the reaction, which later 
turned to greenish-yellow towards the end of the reaction (after a total time period of $12 \mathrm{~h}$ ). The reaction mixture was then quenched with deionized water $(10 \mathrm{ml})$ and contents were vigorously stirred for $24 \mathrm{~h}$ at r.t. under an argon atmosphere. The reaction mixture was extracted with dichloromethane $(50 \mathrm{ml})$ and deionized water $(50 \mathrm{ml})$. The aqueous phase was washed with additional dichloromethane $(2 \times 25 \mathrm{ml})$. The combined organic phases were washed with saturated sodium bicarbonate solution $(50 \mathrm{ml})$ followed by saturated brine solution $(2 \times 25 \mathrm{ml})$ and was dried over anhydrous $\mathrm{Na}_{2} \mathrm{SO}_{4}$. It was filtered and evaporated to dryness under a reduced pressure to obtain an off-white amorphous powder. This was purified by flash chromatography on silica gel (ethyl acetate/hexane $=3: 7)$ to obtain the amide $47(0.159 \mathrm{~g}, 82 \%)$ as an offwhite amorphous powder, $\mathrm{R}_{f}=0.29$ (ethyl acetate/hexane $=3: 7$ ), mp $213.5-215{ }^{\circ} \mathrm{C}$ [Found (EI): $\mathrm{M}^{+\bullet}$ 346.2044, $\mathrm{C}_{23} \mathrm{H}_{26} \mathrm{~N}_{2} \mathrm{O}(\mathrm{M})$ requires $M$ 346.2040]; IR $\left(\mathrm{CHCl}_{3}\right)$ 3359, 2932, 2867, 1660, 1652, 1645, 1532 , 1496, 1470, 1454, 1416, 1368, 1334, 1321, 1250, 1179, $741 \mathrm{~cm}^{-1} ;{ }^{1} \mathrm{H}$ NMR (400 MHz, $\left.\mathrm{CDCl}_{3}\right) \delta 1.14(\mathrm{~s}$, $3 \mathrm{H}), 1.85(\mathrm{~s}, 3 \mathrm{H}), 1.45-1.60(\mathrm{~m}, 1 \mathrm{H}), 1.962 .10(\mathrm{~m}, 1 \mathrm{H}), 2.35-2.55(\mathrm{~m}, 1 \mathrm{H}), 2.63-2.82(\mathrm{~m}, 2 \mathrm{H}), 2.82-2.95(\mathrm{~m}$, $1 \mathrm{H}), 3.05-3.20(\mathrm{~m}, 1 \mathrm{H}), 3.90-4.06(\mathrm{~m}, 1 \mathrm{H}), 5.15-5.30$ (broad d, $J=17.1 \mathrm{~Hz}, 1 \mathrm{H}), 5.27-5.47$ (broad d, $J=$ 17.1 Hz, 1H), 5.38-5.67 (broad s, 1H), 6.88-7.03 (m, 2H), 7.10-7.20 (m, 2H), 7.21-7.30 (m, 4H), 7.45-7.68 $(\mathrm{m}, 1 \mathrm{H}) ;{ }^{13} \mathrm{C}$ NMR $\left(100.61 \mathrm{MHz}, \mathrm{CDCl}_{3}\right) \delta 19.7\left(\mathrm{CH}_{2}\right), 22.9\left(\mathrm{CH}_{2}\right), 24.4\left(\mathrm{CH}_{3}\right), 26.6\left(\mathrm{CH}_{3}\right), 39.3\left(\mathrm{CH}_{2}\right)$, $42.4(\mathrm{C}), 43.4\left(\mathrm{CH}_{2}\right), 46.7\left(\mathrm{CH}_{2}\right), 108.4(\mathrm{C}), 109.4(\mathrm{CH}), 117.9(\mathrm{CH}), 119.4(\mathrm{CH}), 121.5(\mathrm{CH}), 125.9(\mathrm{CH})$, $127.3(\mathrm{C}), 127.4(\mathrm{CH}), 128.8(\mathrm{CH}), 136.6(\mathrm{C}), 137.8(\mathrm{C}), 140.0(\mathrm{C}), 179.7(\mathrm{C})$; GC-MS retention time 20.63 min., $m / z$ (EI) $346\left(\mathrm{M}^{+\bullet}, 100 \%\right), 302$ (3), 288 (6), 261 (9), 260 (35), 255 (28), 248 (27), 232 (22.5), 218 (8), 168 (14), 130 (7), 91 (77), 77 (7), 65 (5), 57 (8). The structure of the amide was further confirmed by ${ }^{1} \mathrm{H},{ }^{1} \mathrm{H}$ 2D COSY, ${ }^{1} \mathrm{H},{ }^{13} \mathrm{C} 2 \mathrm{D}$ HSQC and ${ }^{1} \mathrm{H},{ }^{13} \mathrm{C}$ 2D HMBC experiments.

\section{References.}

1. Quesnal, Y.; Bidois, L.; Poirier, J. M.; Duhamel, L. Synlett 1998, 413.

2. Kim, S.; Joe, G. H.; Do, J. Y. J. Am. Chem. Soc. 1994, 116, 2000.

3. Fristad, W. E.; Bailey, T. R.; Paquette, L. A. J.Org. Chem. 1990, 55, 3028.

4. Christiansen, G. D.; Lightner, D. A. J. Org. Chem. 1971, 36, 948. Krapcho, A. P; Donn, R. J. Org. Chem. 1965, 30641 .

5. Gayet, A.; Bertilsson, S.; Andersson, P. G. Org. Lett. 2002, 4, 3777. 
${ }^{1} \mathrm{H}$ NMR Spectrum of 21; Solvent: $\mathrm{CDCl}_{3} ; 400.13 \mathrm{MHz}$; Instrument: Bruker DPX400 Spectrometer
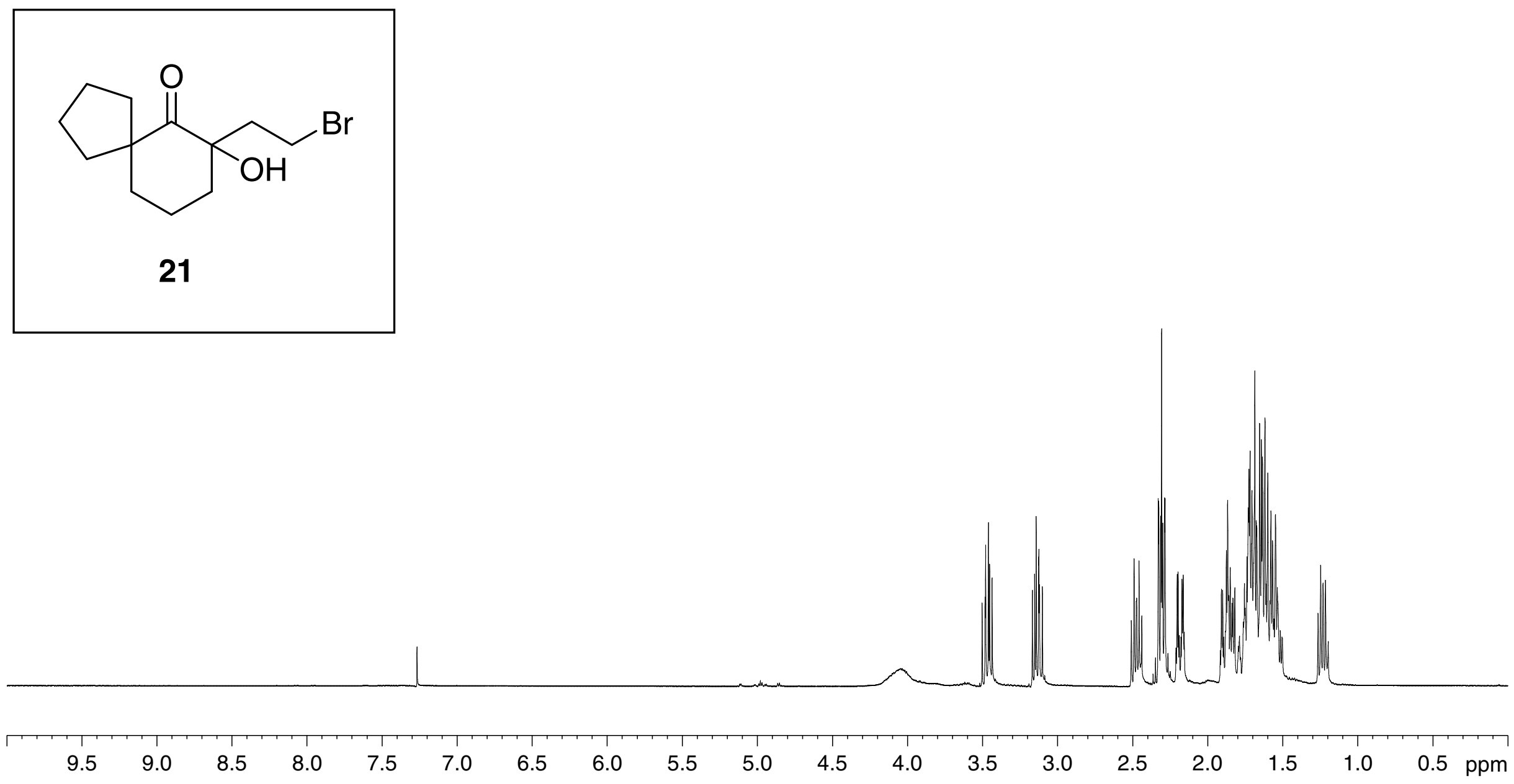
${ }^{13} \mathrm{C}$ NMR (JMOD) Spectrum of 21; Solvent: $\mathrm{CDCl}_{3} ; 100.61 \mathrm{MHz}$; Instrument: Bruker DPX400 Spectrometer
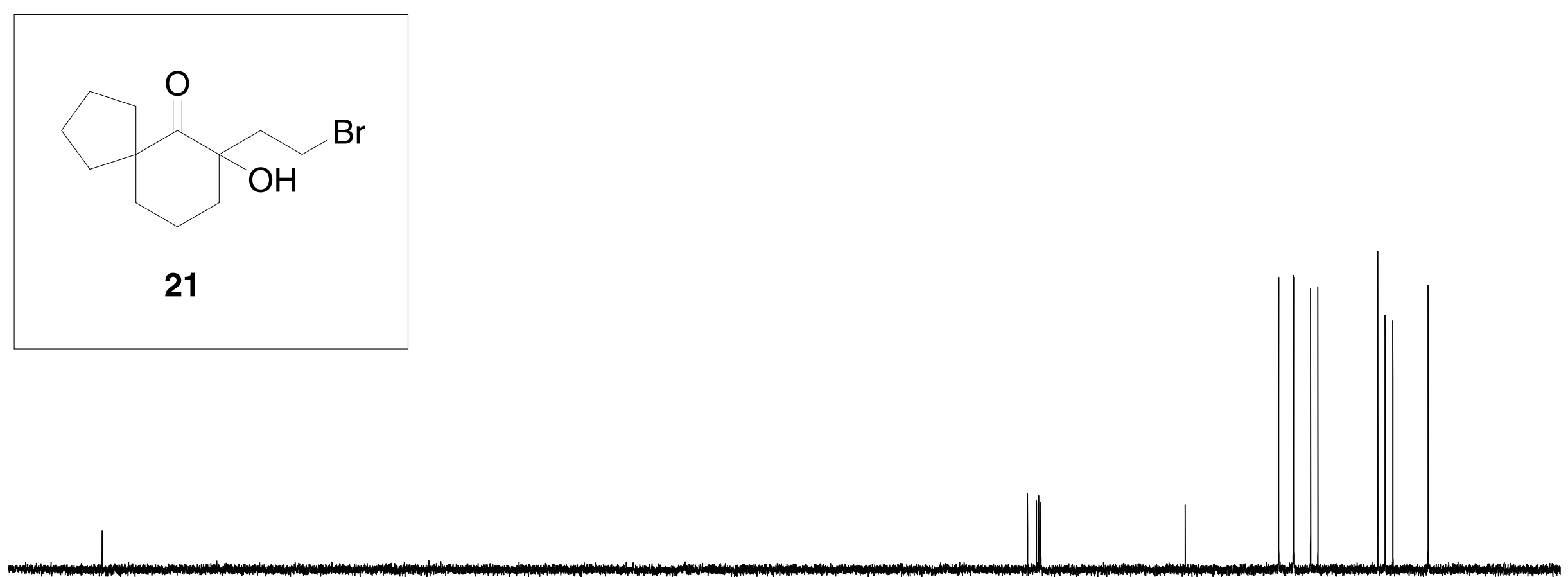
${ }^{1} \mathrm{H}$ NMR Spectrum of 22a; Solvent: $\mathrm{CDCl}_{3} ; 400.13 \mathrm{MHz}$; Instrument: Bruker DPX400 Spectrometer

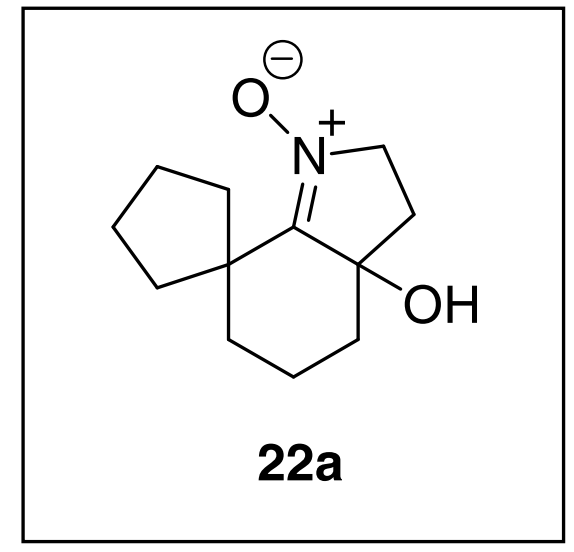


${ }^{13} \mathrm{C}$ NMR (JMOD) Spectrum of 22a; Solvent: $\mathrm{CDCl}_{3}$; $100.61 \mathrm{MHz}$; Instrument: Bruker DPX400 Spectrometer

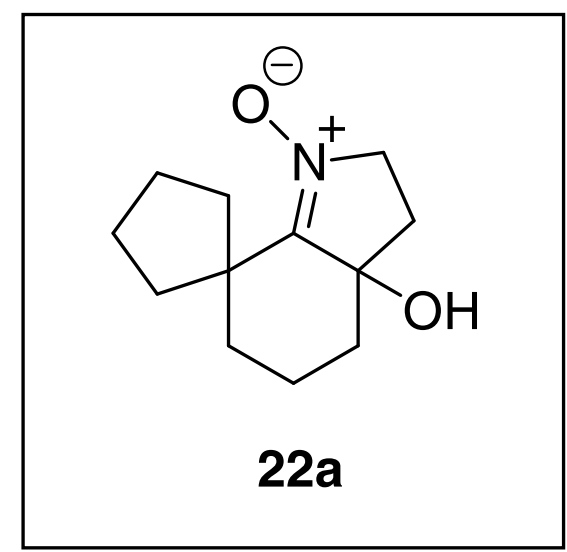


${ }^{1} \mathrm{H}$ NMR Spectrum of 23; Solvent: $\mathrm{CDCl}_{3}$; $400.13 \mathrm{MHz}$; Instrument: Bruker DPX400 Spectrometer
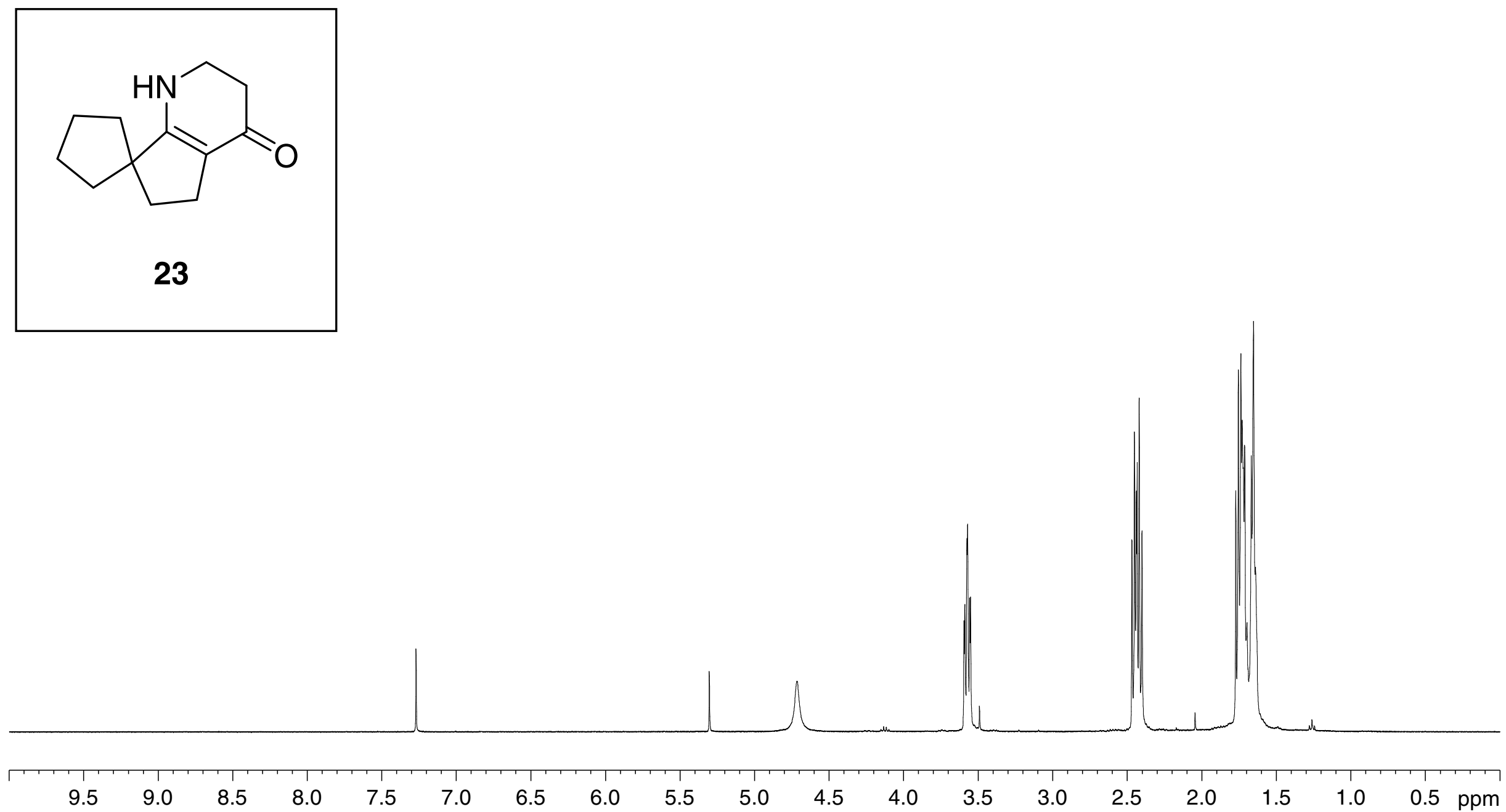
${ }^{13}$ C NMR (JMOD) Spectrum of 23; Solvent: $\mathrm{CDCl}_{3} ; 100.61 \mathrm{MHz}$; Instrument: Bruker DPX400 Spectrometer

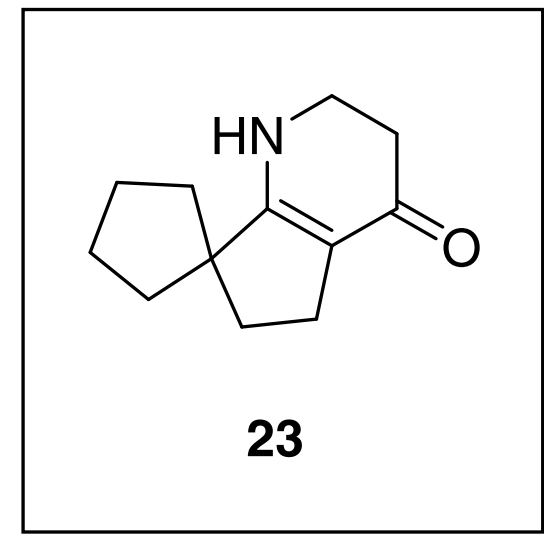

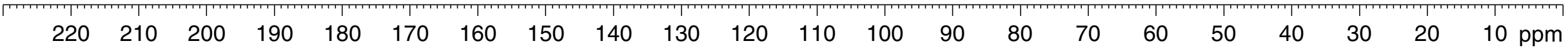


${ }^{1} \mathrm{H}$ NMR Spectrum of 26; Solvent: $\mathrm{CDCl}_{3}$; $400.13 \mathrm{MHz}$; Instrument: Bruker DPX400 Spectrometer
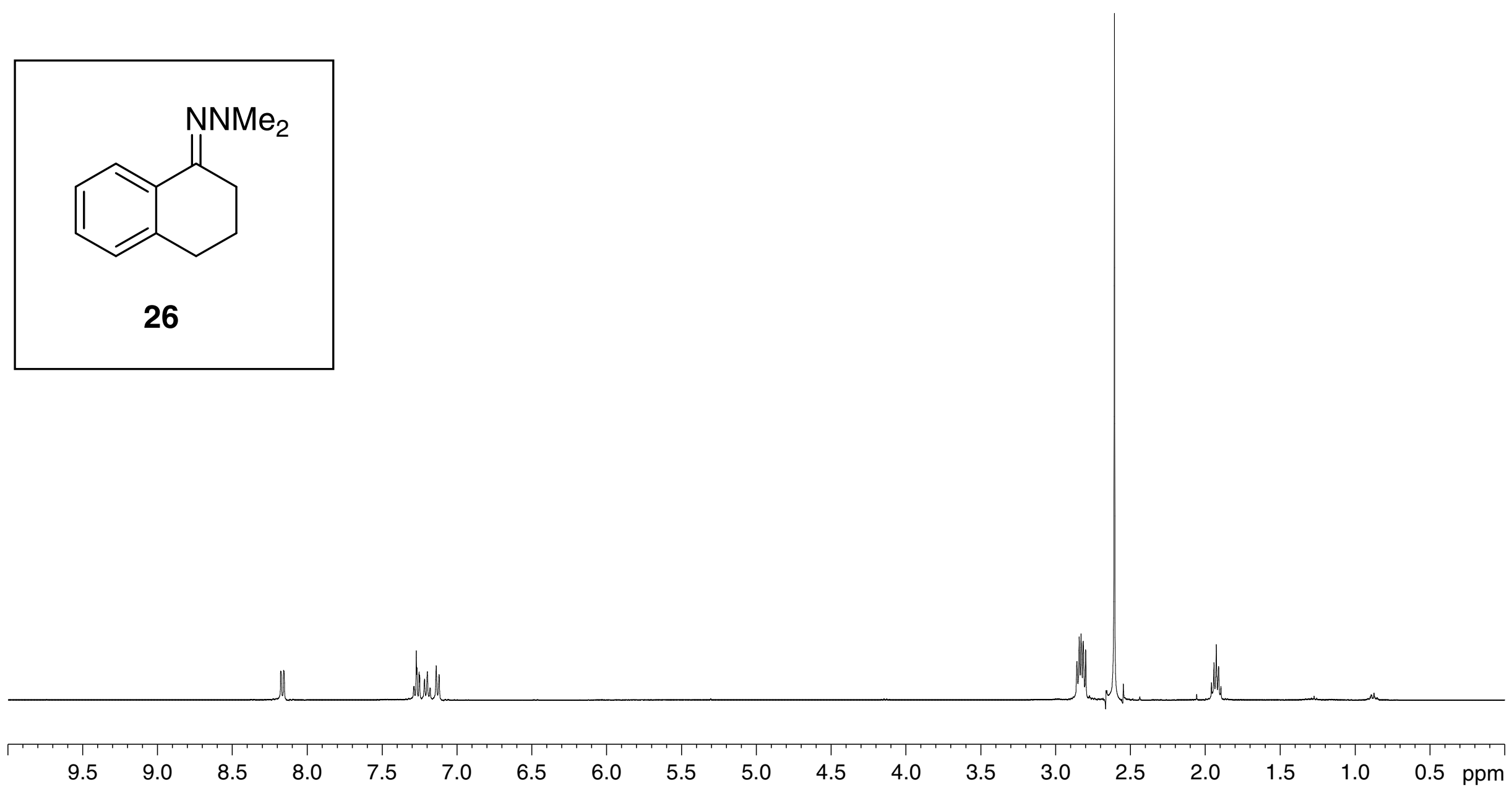
${ }^{13} \mathrm{C}$ NMR (JMOD) Spectrum of 26; Solvent: $\mathrm{CDCl}_{3} ; 100.61 \mathrm{MHz}$; Instrument: Bruker DPX400 Spectrometer

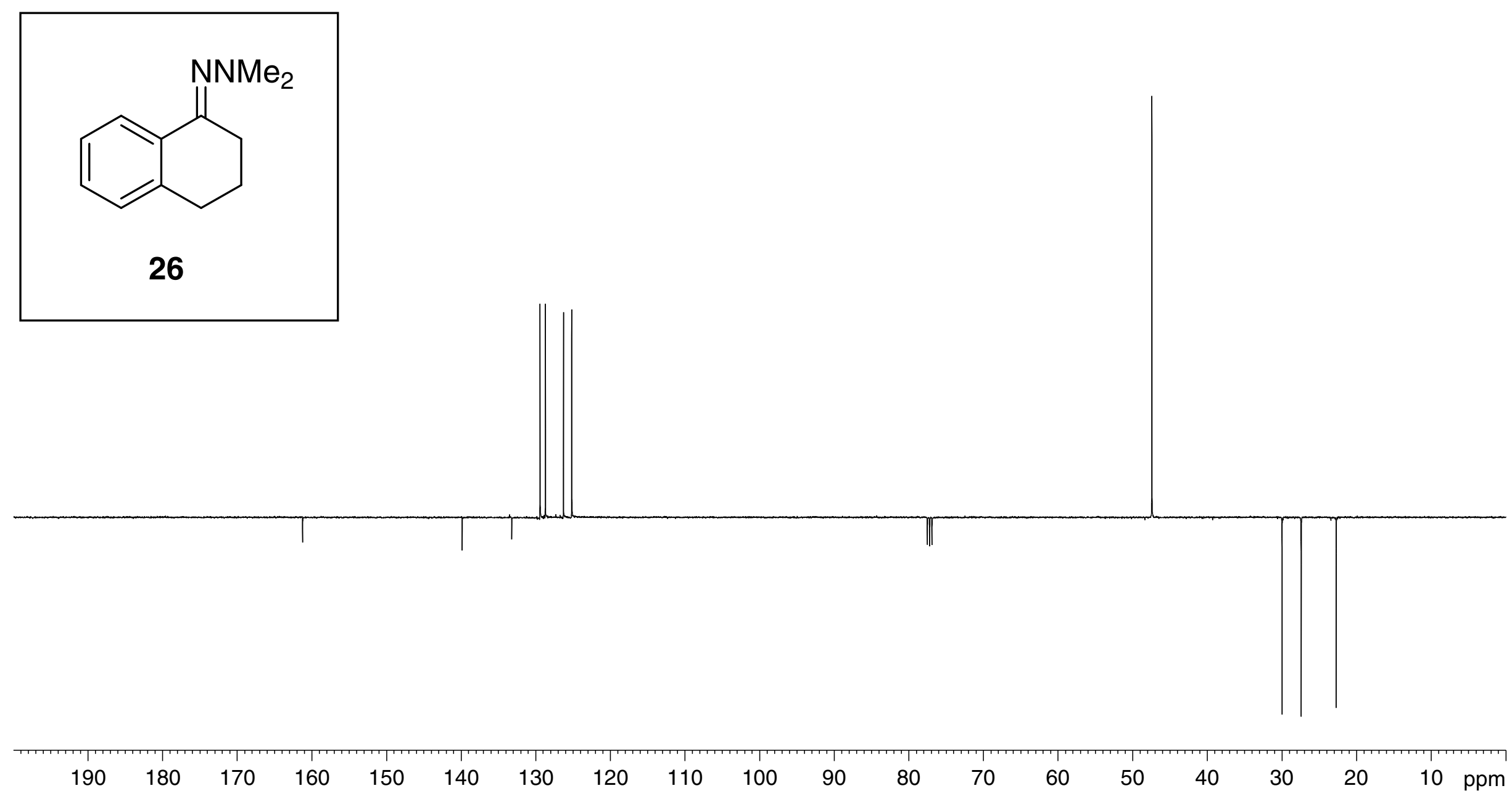


${ }^{1} \mathrm{H}$ NMR Spectrum of 27; Solvent: $\mathrm{CDCl}_{3} ; 400.13 \mathrm{MHz}$; Instrument: Bruker DPX400 Spectrometer
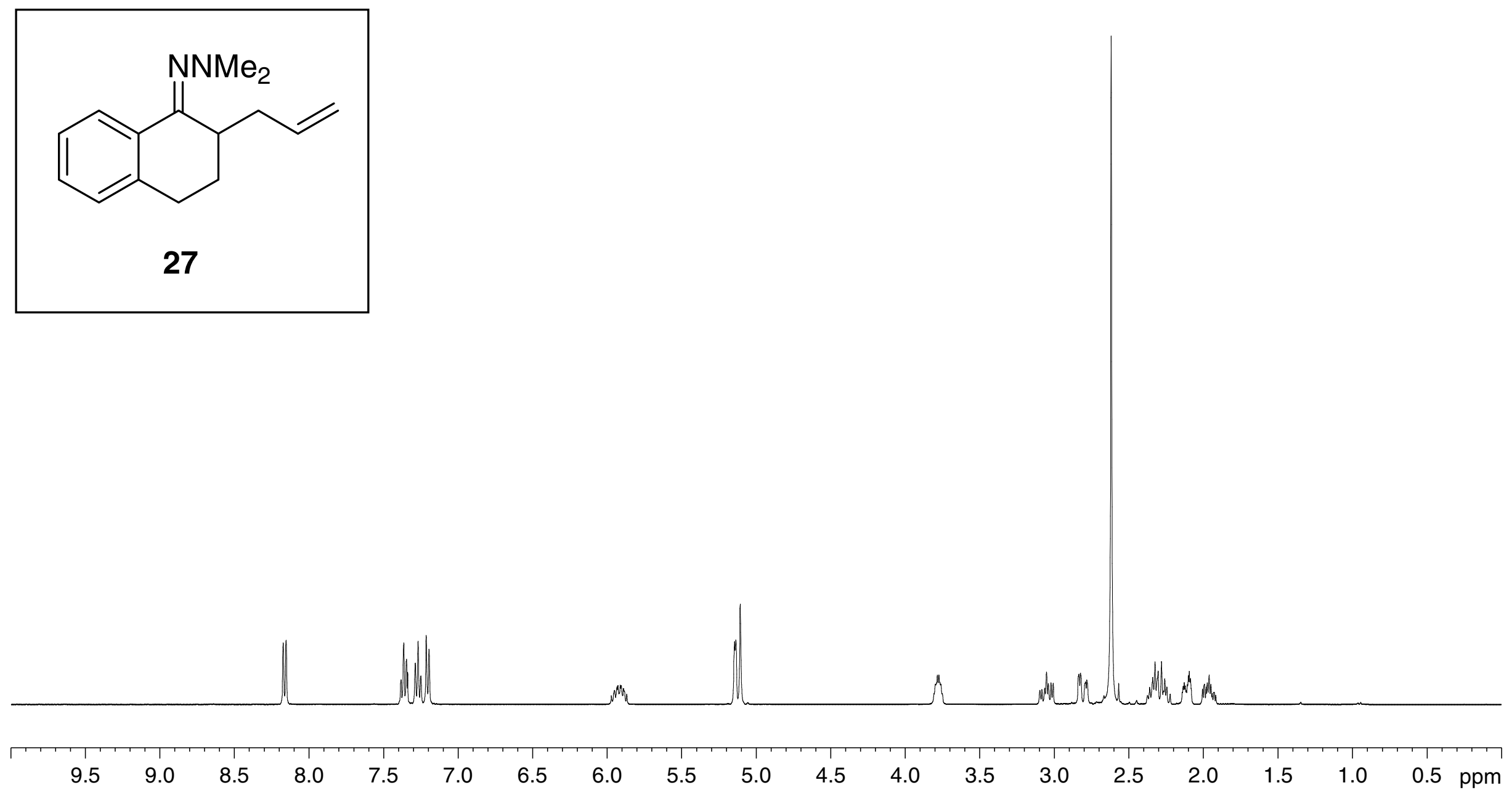
${ }^{13} \mathrm{C}$ NMR (JMOD) Spectrum of 27; Solvent: $\mathrm{CDCl}_{3} ; 100.61 \mathrm{MHz}$; Instrument: Bruker DPX400 Spectrometer

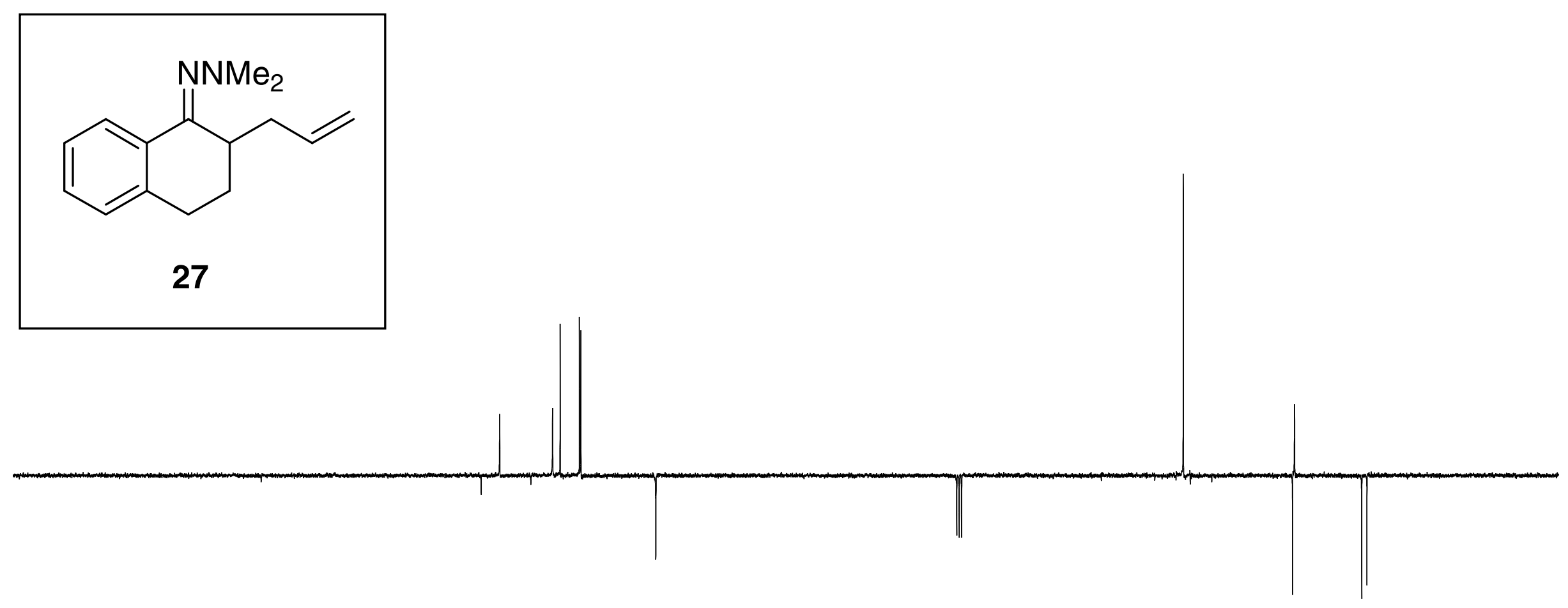

$\begin{array}{lllllllllllllllllllll}190 & 180 & 170 & 160 & 150 & 140 & 130 & 120 & 110 & 100 & 90 & 80 & 70 & 60 & 50 & 40 & 30 & 20 & 10 & \text { ppm }\end{array}$


${ }^{1} \mathrm{H}$ NMR Spectrum of 28; Solvent: $\mathrm{CDCl}_{3}$; $400.13 \mathrm{MHz}$; Instrument: Bruker DPX400 Spectrometer
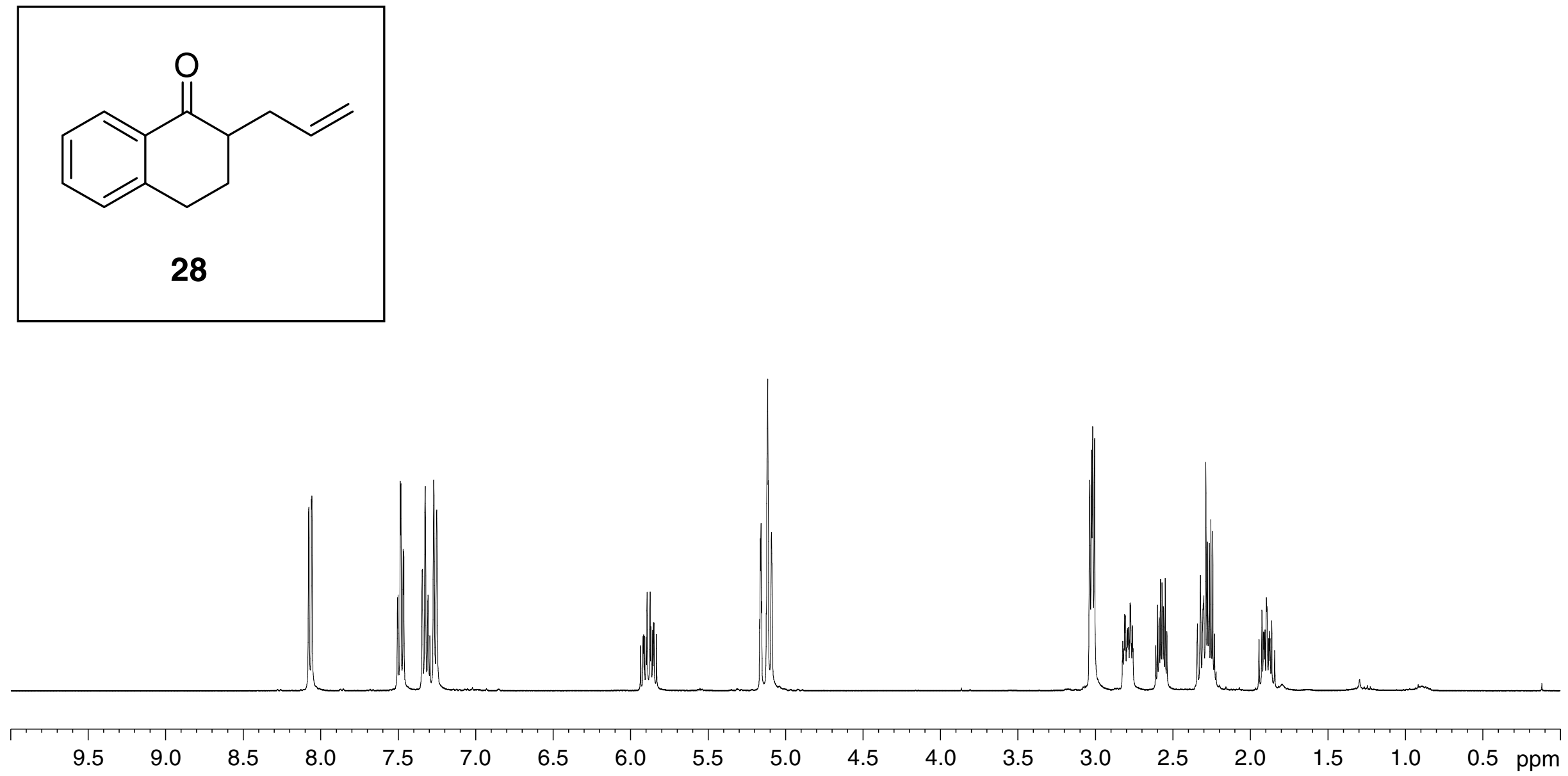
${ }^{13} \mathrm{C}$ NMR (JMOD) Spectrum of 28; Solvent: $\mathrm{CDCl}_{3} ; 100.61 \mathrm{MHz}$; Instrument: Bruker DPX400 Spectrometer

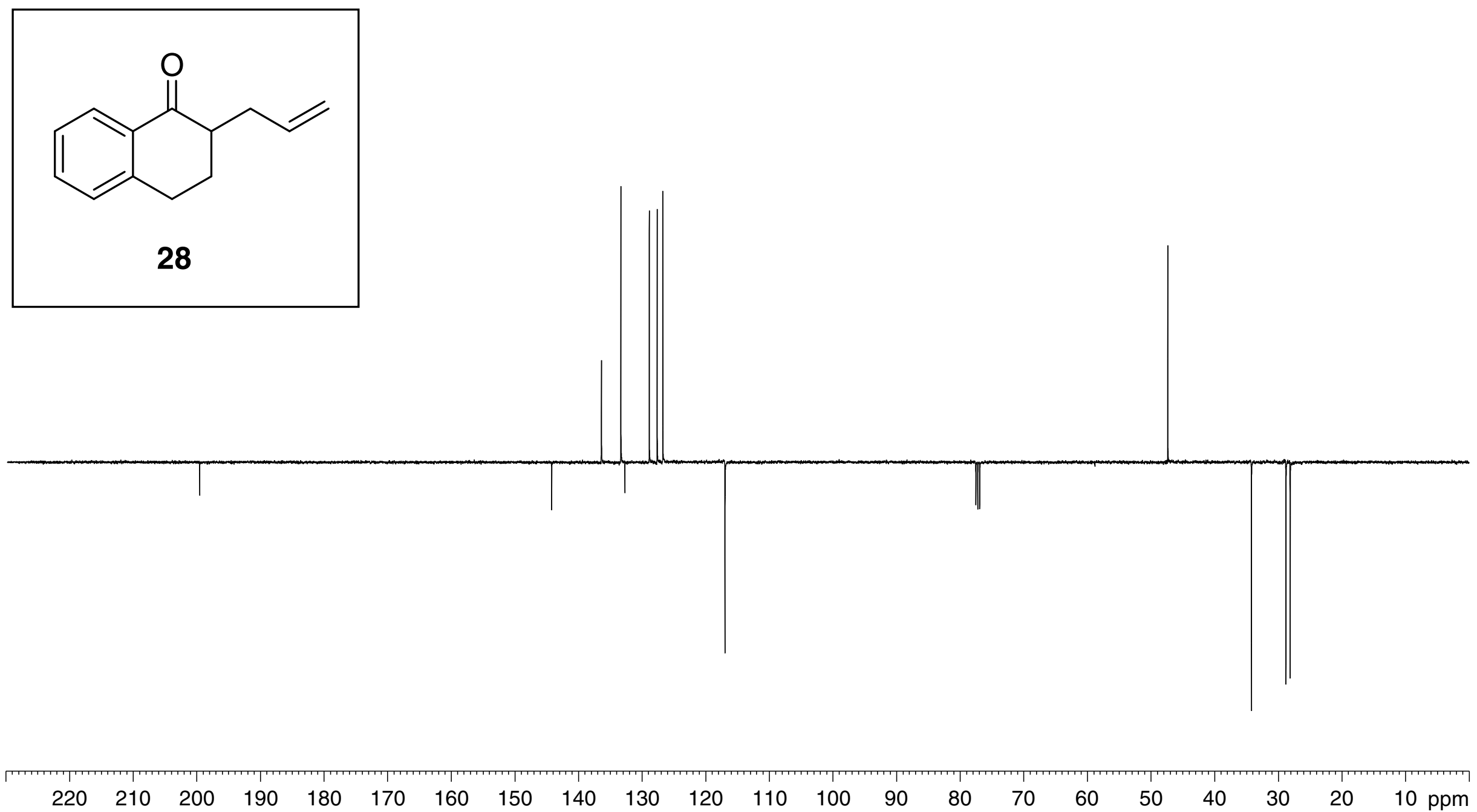


${ }^{1} \mathrm{H}$ NMR Spectrum of 29; Solvent: $\mathrm{CDCl}_{3}$; $400.13 \mathrm{MHz}$; Instrument: Bruker DPX400 Spectrometer
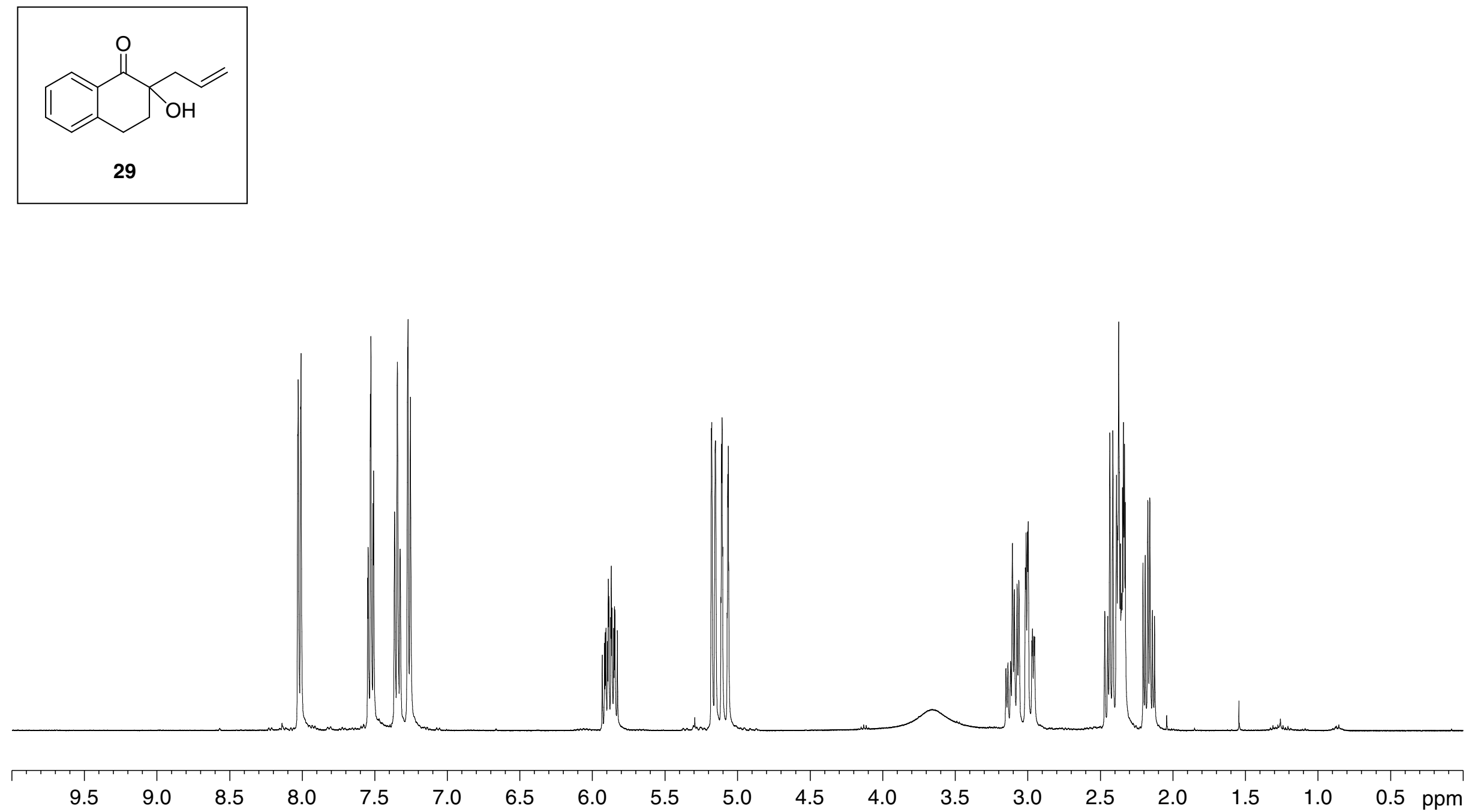
${ }^{13}$ C NMR (JMOD) Spectrum of 29; Solvent: $\mathrm{CDCl}_{3} ; 100.61 \mathrm{MHz}$; Instrument: Bruker DPX400 Spectrometer

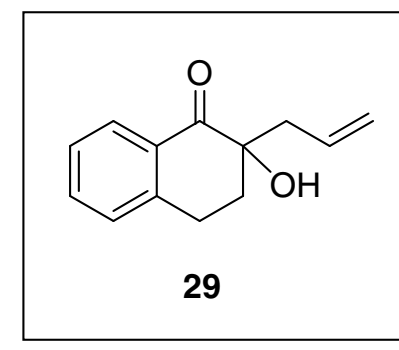

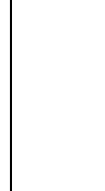

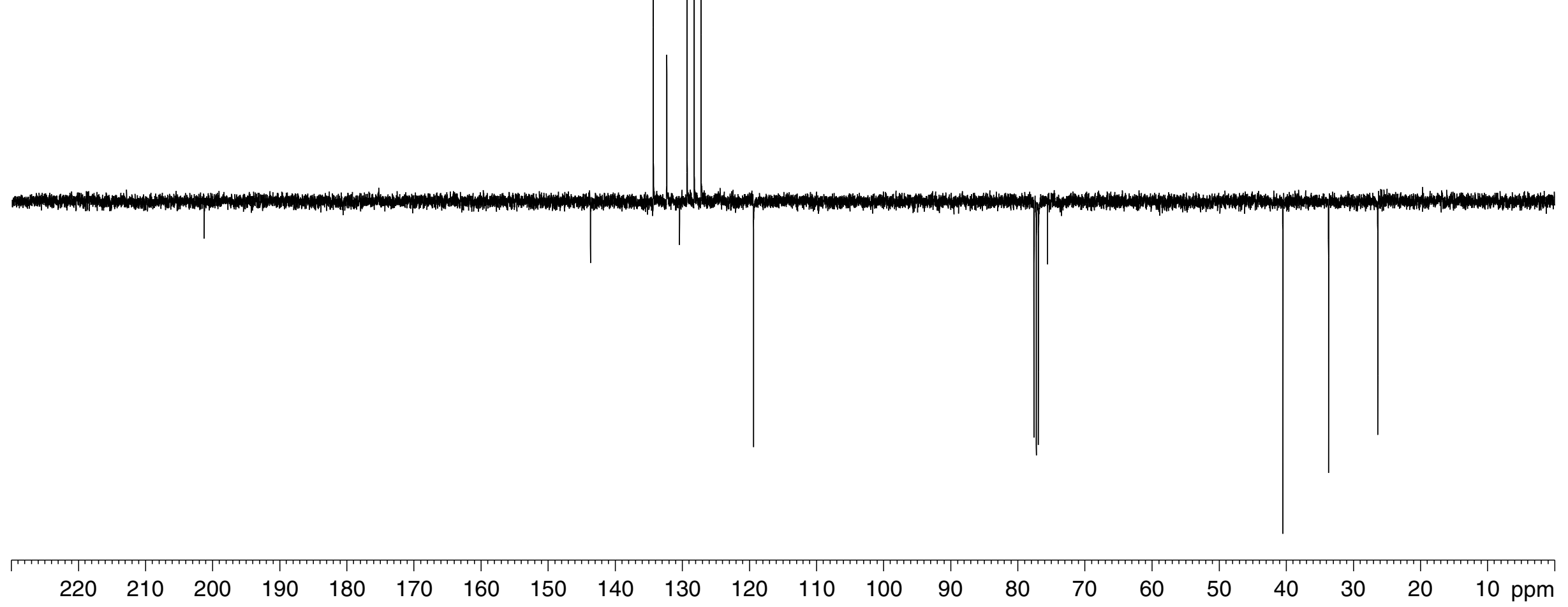


${ }^{1} \mathrm{H}$ NMR Spectrum of 30; Solvent: $\mathrm{CDCl}_{3} ; 400.13 \mathrm{MHz}$; Instrument: Bruker DPX400 Spectrometer
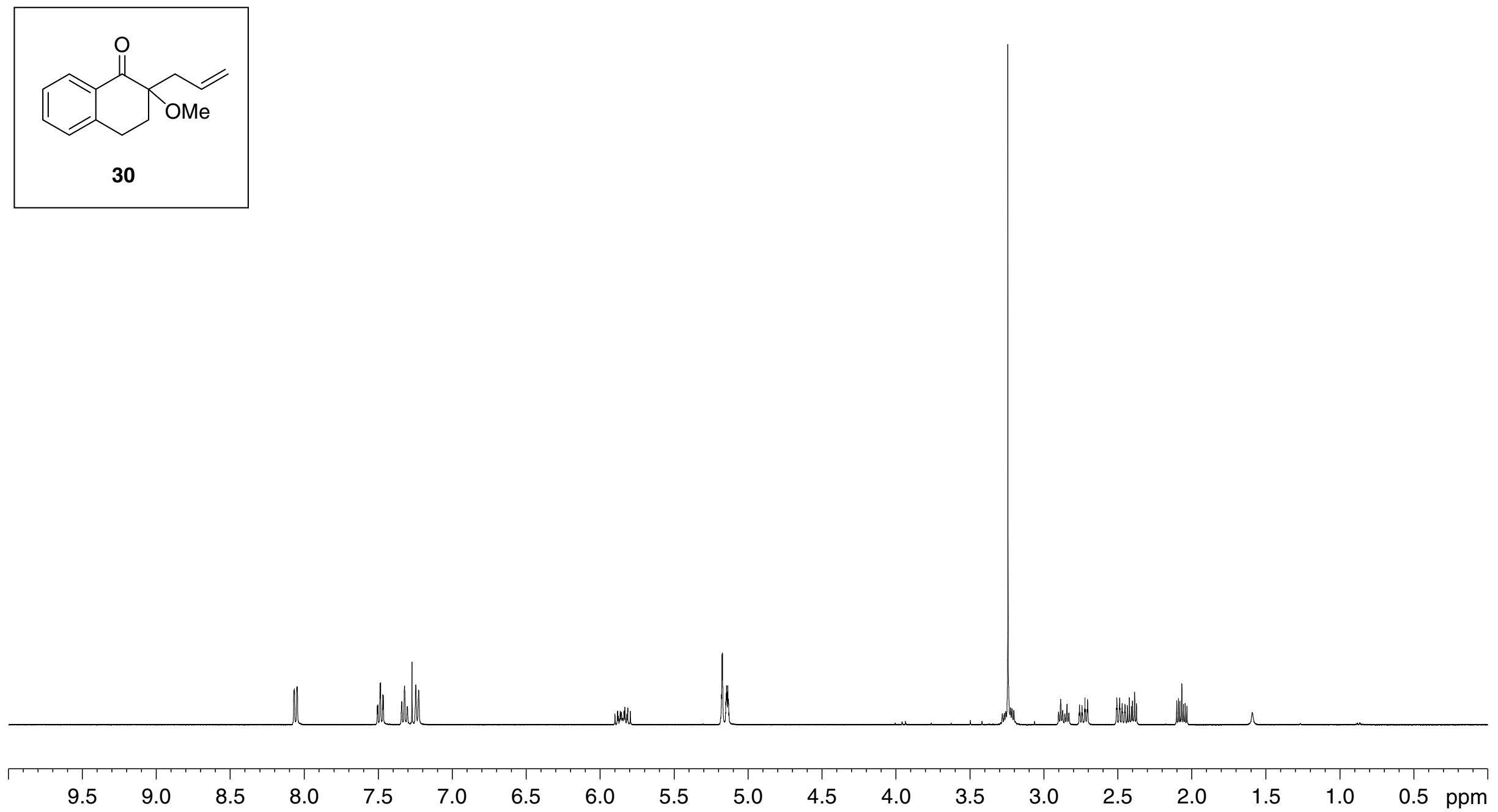
${ }^{13}$ C NMR (JMOD) Spectrum of 30; Solvent: $\mathrm{CDCl}_{3} ; 100.61 \mathrm{MHz}$; Instrument: Bruker DPX400 Spectrometer
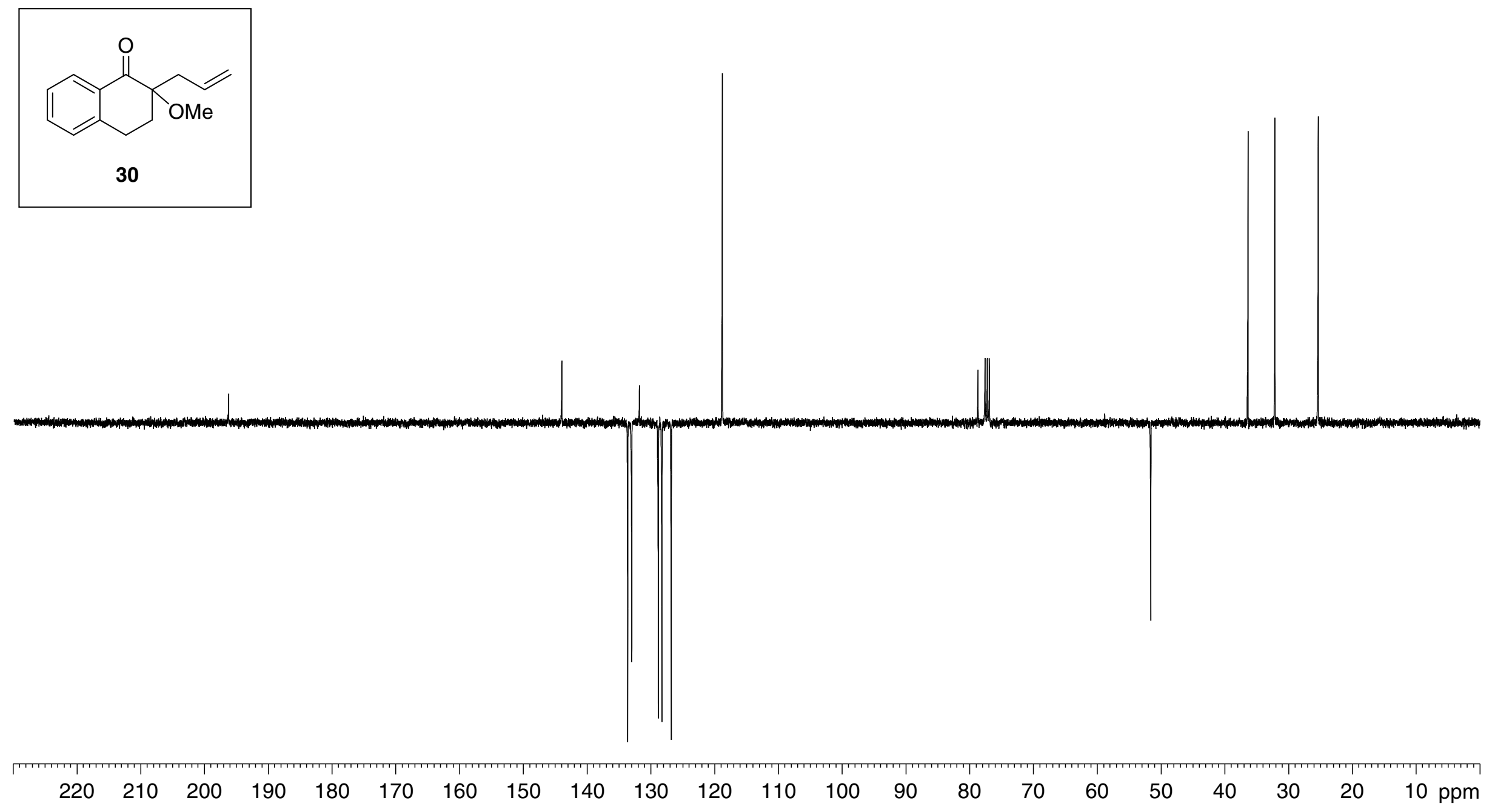
${ }^{1} \mathrm{H}$ NMR Spectrum of diastereoisomer 1 of 31; Solvent: $\mathrm{CDCl}_{3}$; $400.13 \mathrm{MHz}$; Instrument: Bruker DPX400 Spectrometer
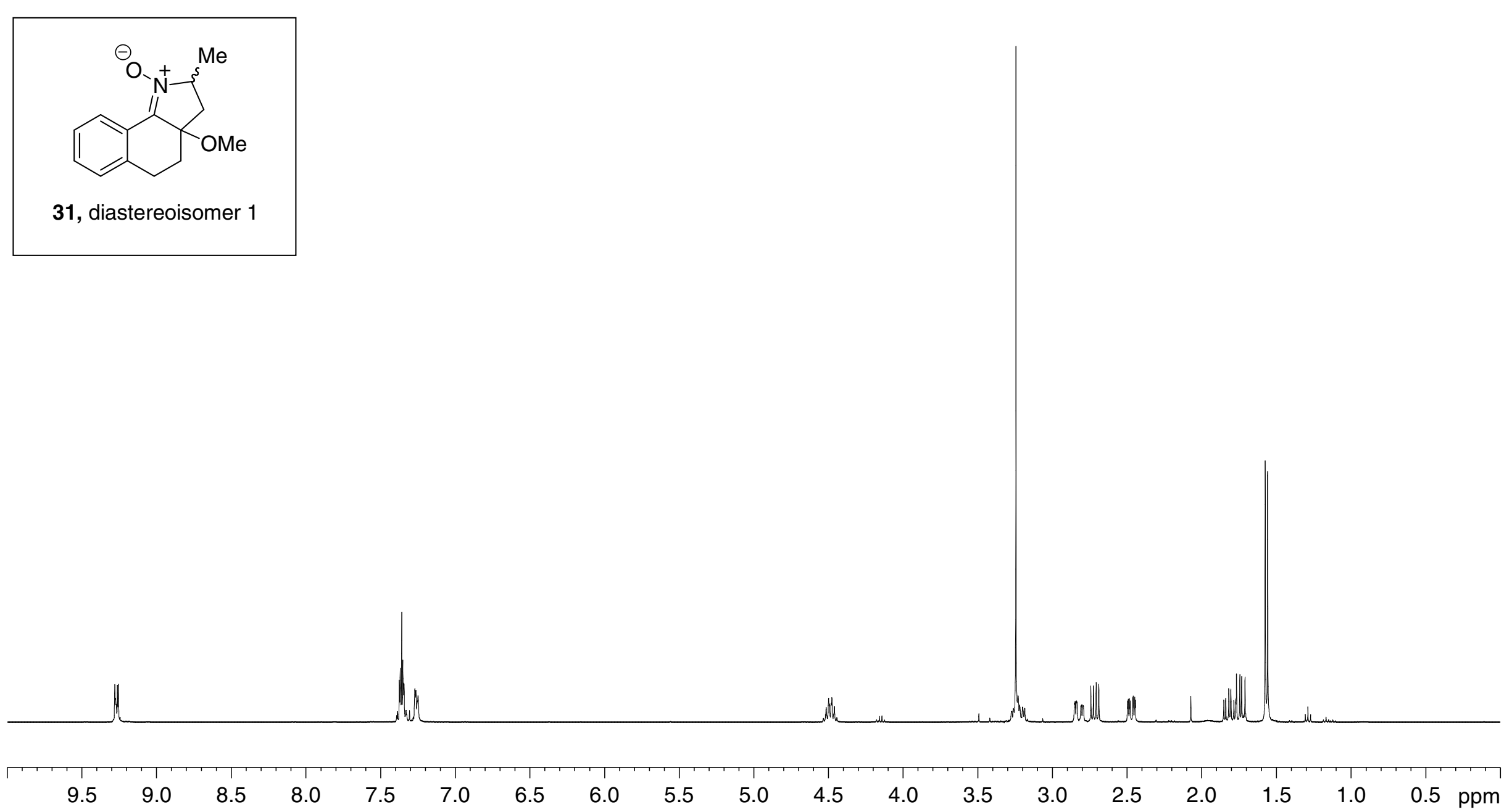
${ }^{13} \mathrm{C}$ NMR (JMOD) Spectrum of diastereoisomer 1 of 31; Solvent: $\mathrm{CDCl}_{3}$; $100.61 \mathrm{MHz}$; Instrument: Bruker DPX400

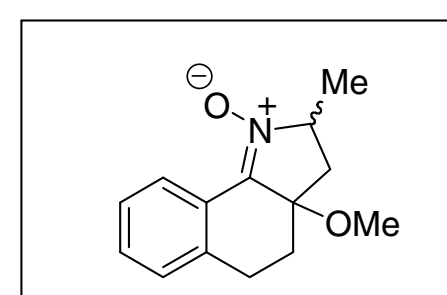

31, diastereoisomer 1

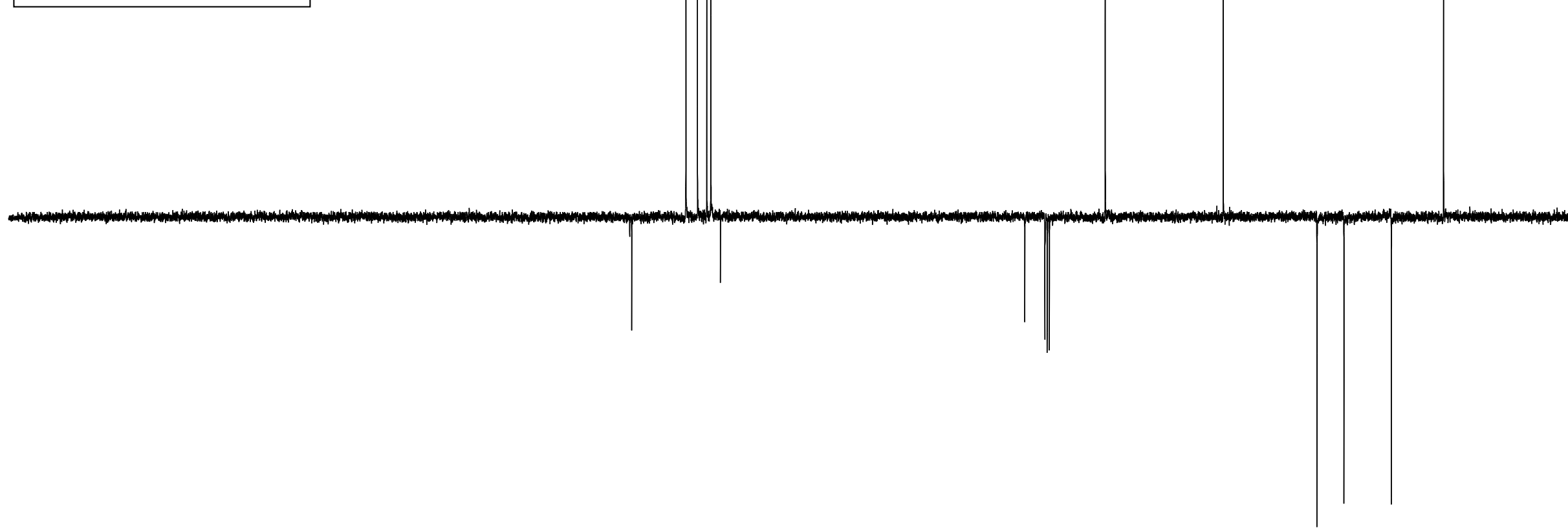


${ }^{1} \mathrm{H}$ NMR Spectrum of diastereoisomer 2 of 31; Solvent: $\mathrm{CDCl}_{3}$; $400.13 \mathrm{MHz}$; Instrument: Bruker DPX400 Spectrometer

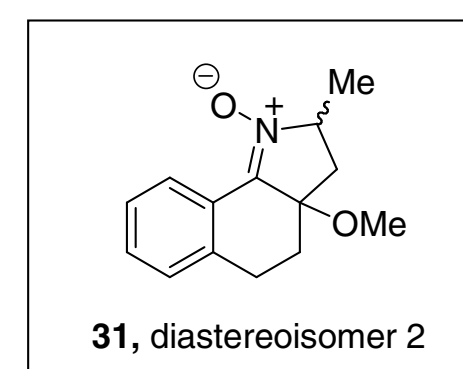

31, diastereoisomer 2

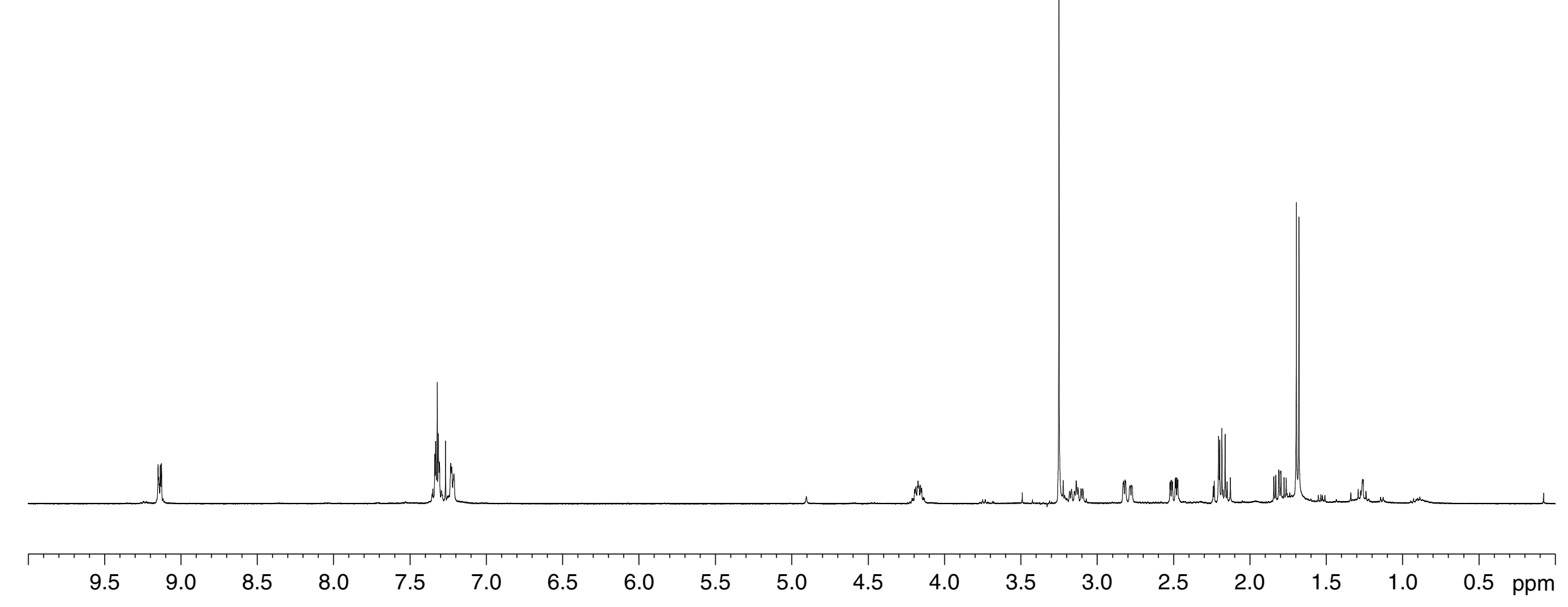


${ }^{13} \mathrm{C}$ NMR (JMOD) Spectrum of diastereoisomer 2 of 31; Solvent: $\mathrm{CDCl}_{3} ; 100.61 \mathrm{MHz}$; Instrument: Bruker DPX400

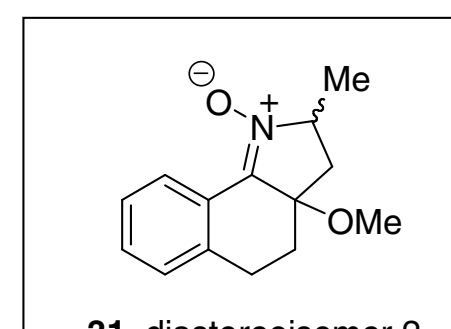

31, diastereoisomer 2

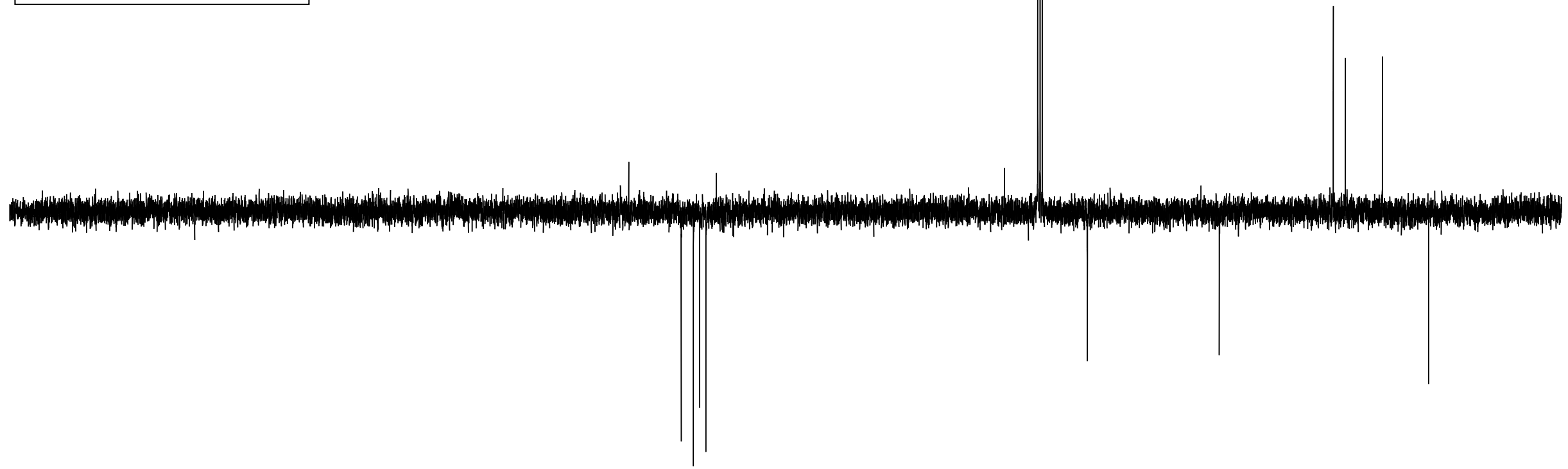

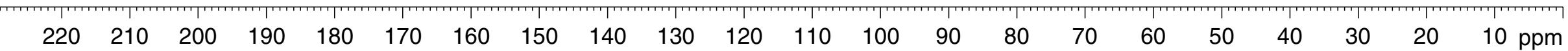


${ }^{1} \mathrm{H}$ NMR Spectrum of 32; Solvent: $\mathrm{CDCl}_{3}$; $400.13 \mathrm{MHz}$; Instrument: Bruker DPX400 Spectrometer
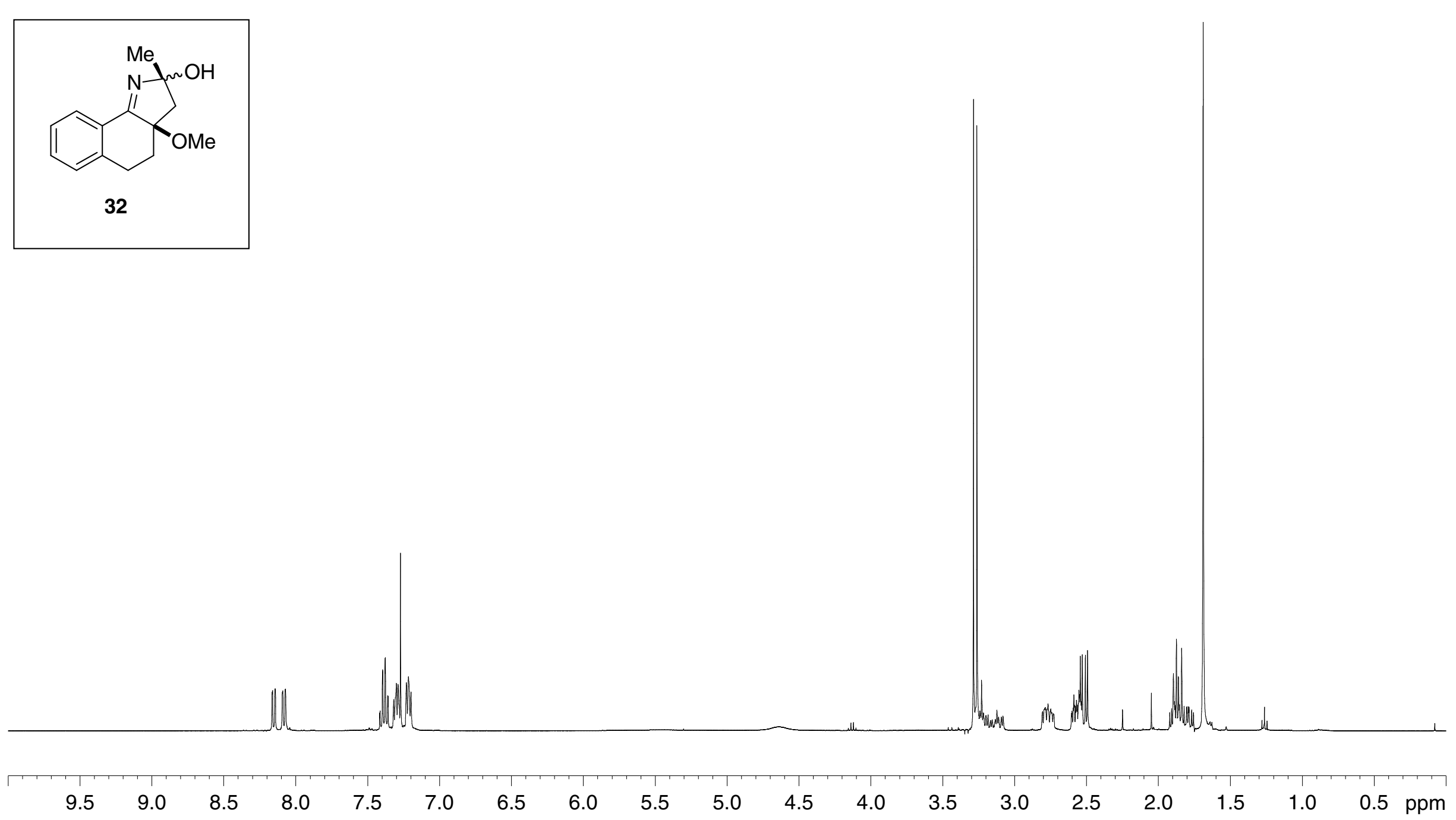
${ }^{13} \mathrm{C}$ NMR (JMOD) Spectrum of 32; Solvent: $\mathrm{CDCl}_{3} ; 100.61 \mathrm{MHz}$; Instrument: Bruker DPX400 Spectrometer
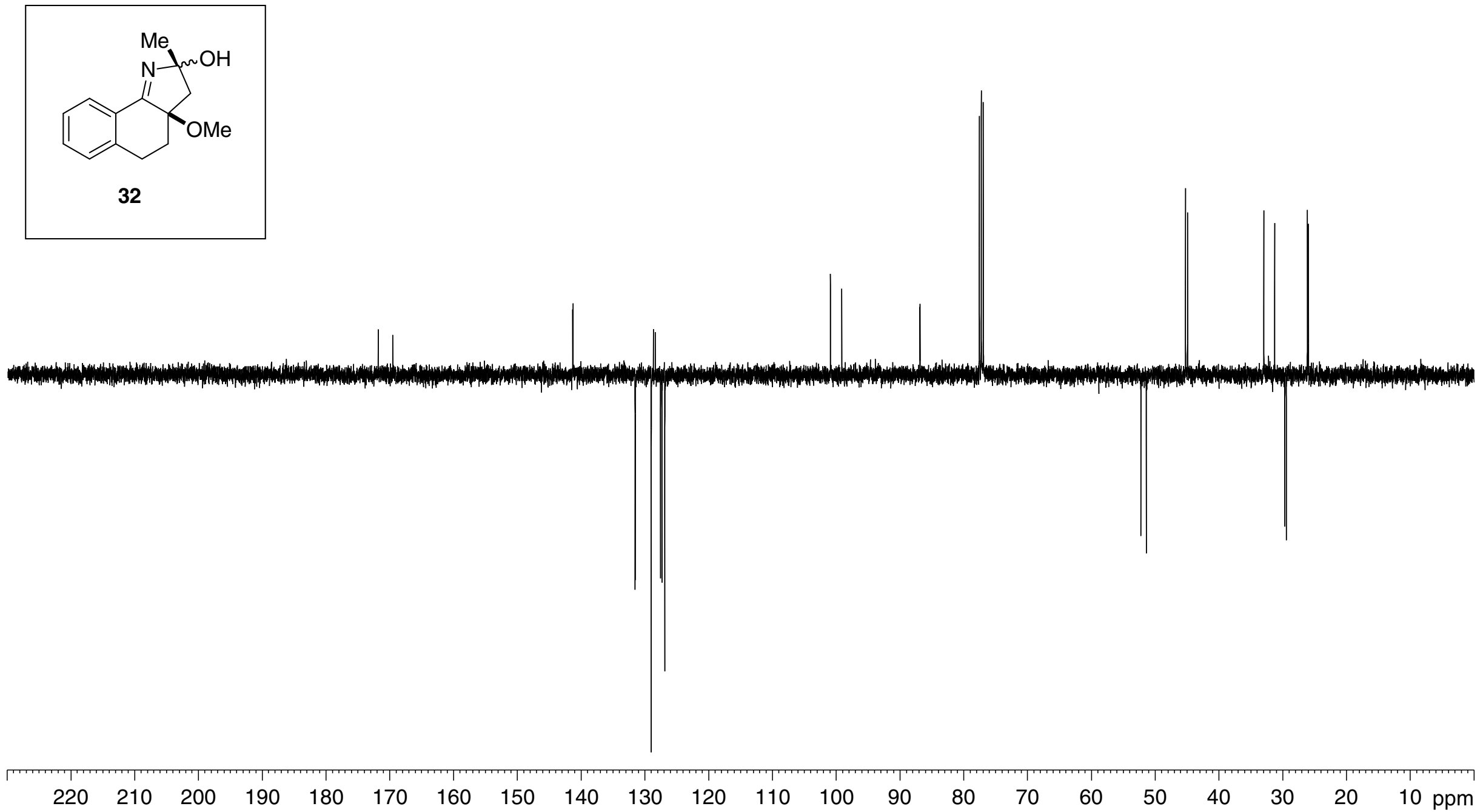
${ }^{1} \mathrm{H}$ NMR Spectrum of 38; Solvent: $\mathrm{CDCl}_{3}$; $400.13 \mathrm{MHz}$; Instrument: Bruker DPX400 Spectrometer
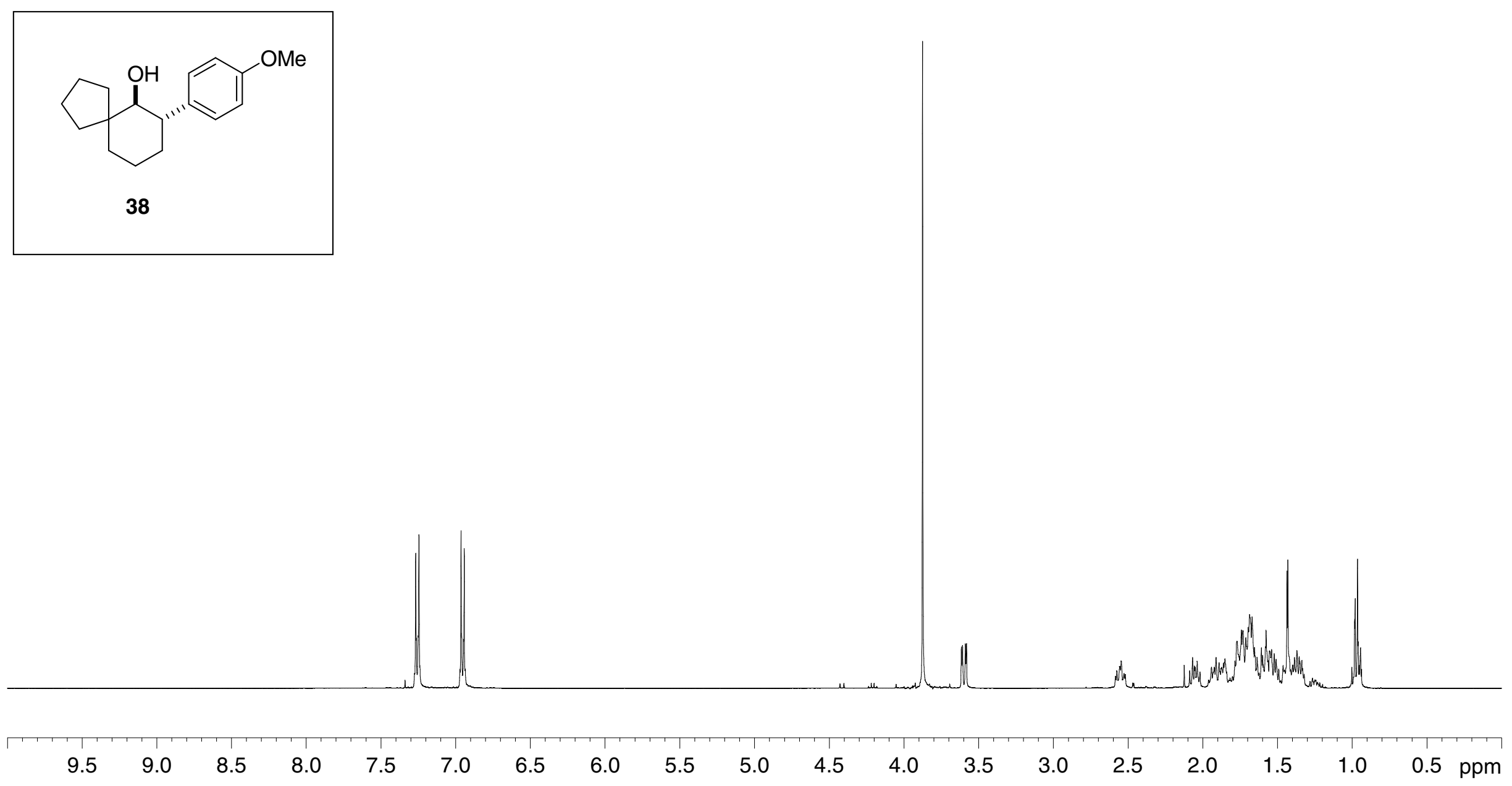
${ }^{13}$ C NMR (JMOD) Spectrum of 38; Solvent: $\mathrm{CDCl}_{3} ; 100.61 \mathrm{MHz}$; Instrument: Bruker DPX400 Spectrometer

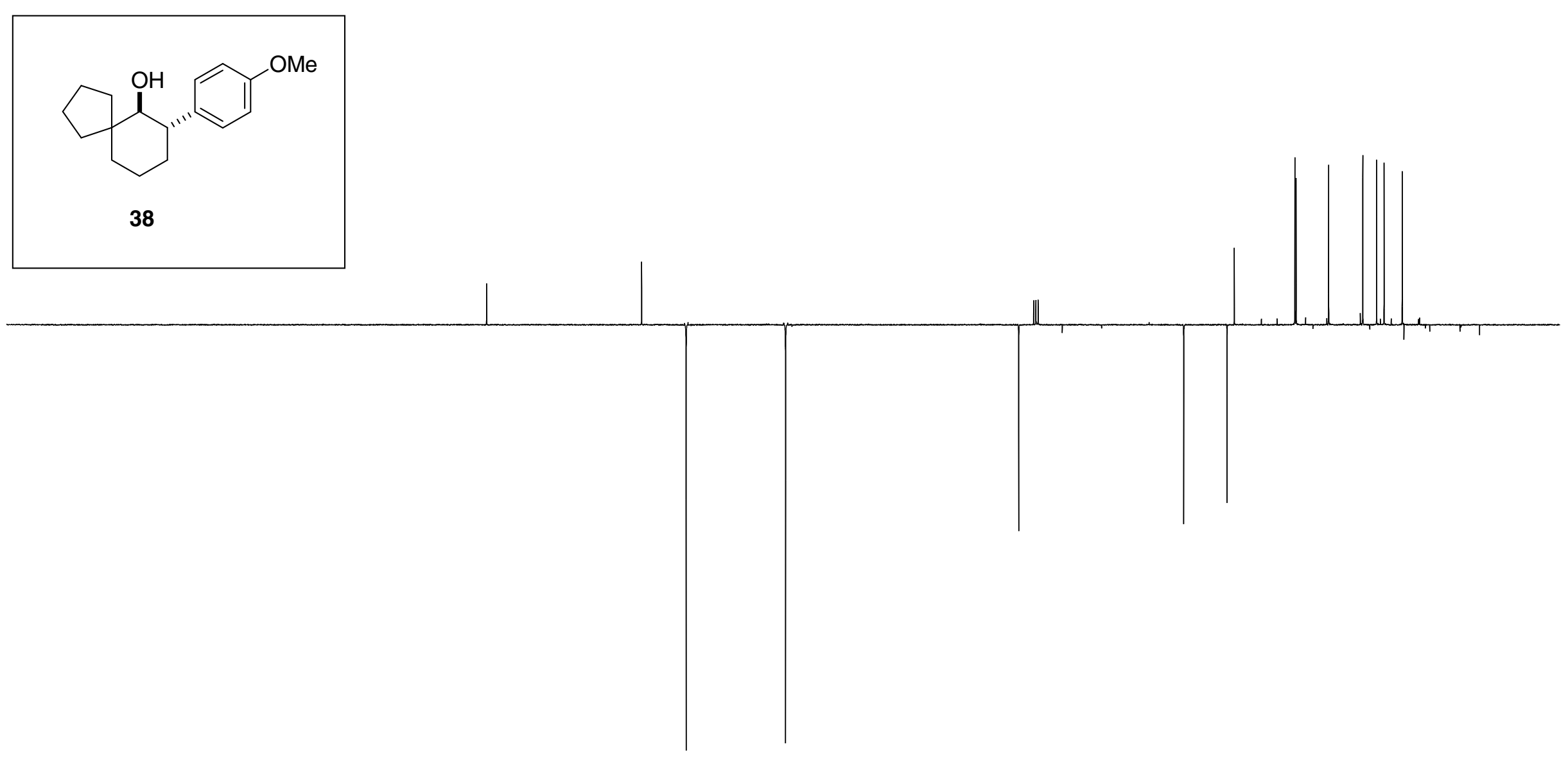


${ }^{1} \mathrm{H}$ NMR Spectrum of 39; Solvent: $\mathrm{CDCl}_{3}$; $400.13 \mathrm{MHz}$; Instrument: Bruker DPX400 Spectrometer
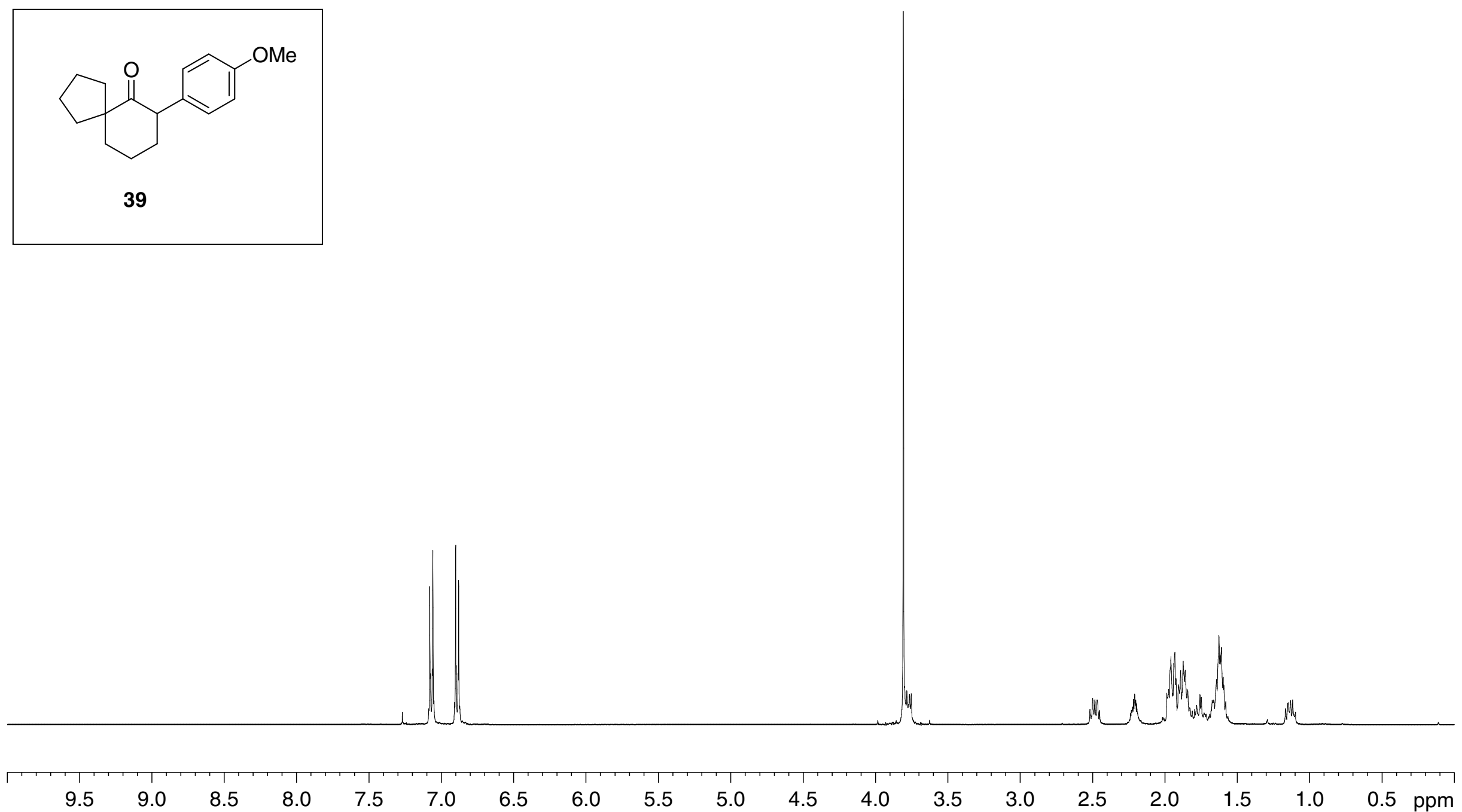
${ }^{13}$ C NMR (JMOD) Spectrum of 39; Solvent: $\mathrm{CDCl}_{3} ; 100.61 \mathrm{MHz}$; Instrument: Bruker DPX400 Spectrometer

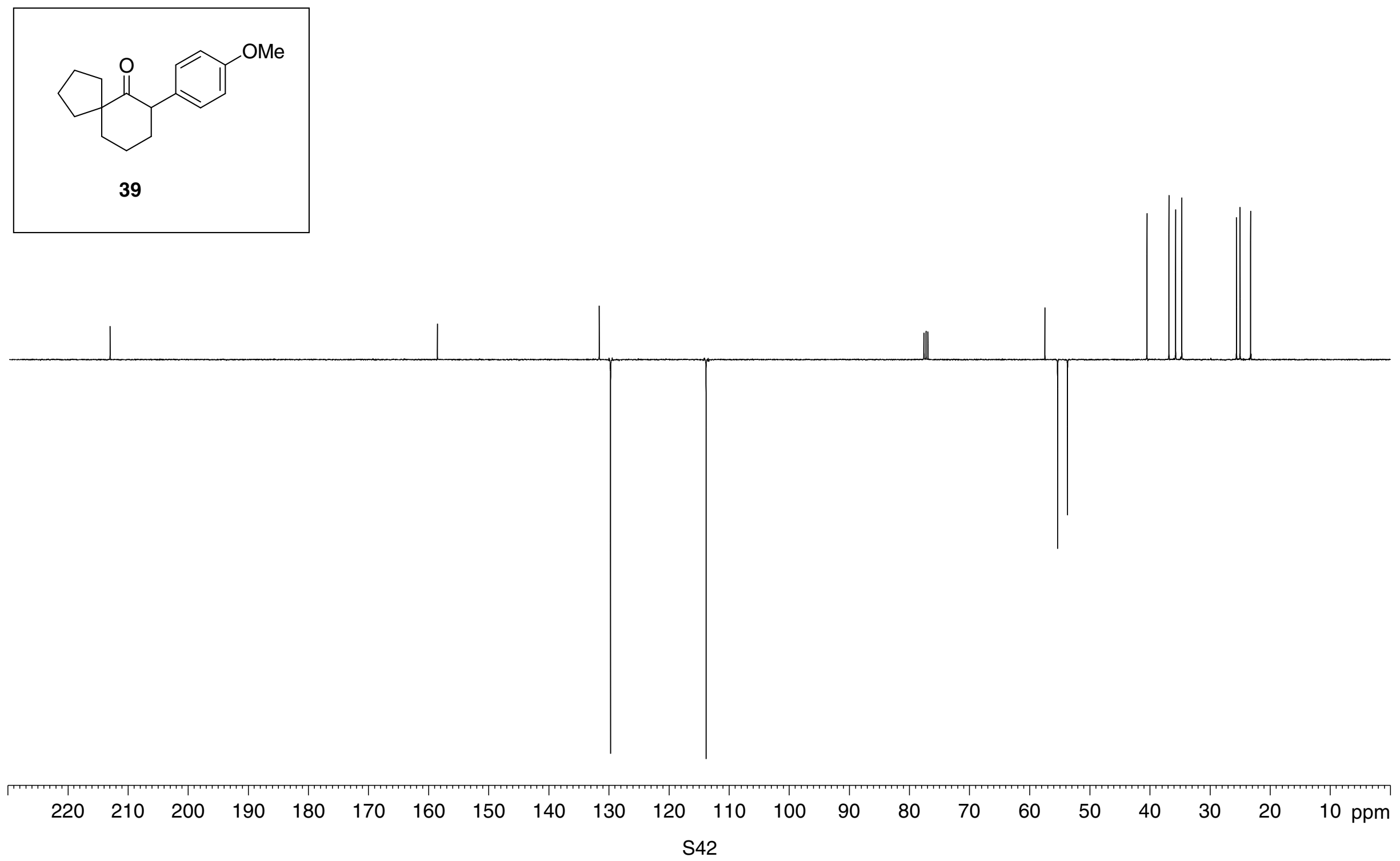


${ }^{1} \mathrm{H}$ NMR Spectrum of 40; Solvent: $\mathrm{CDCl}_{3} ; 400.13 \mathrm{MHz}$; Instrument: Bruker DPX400 Spectrometer
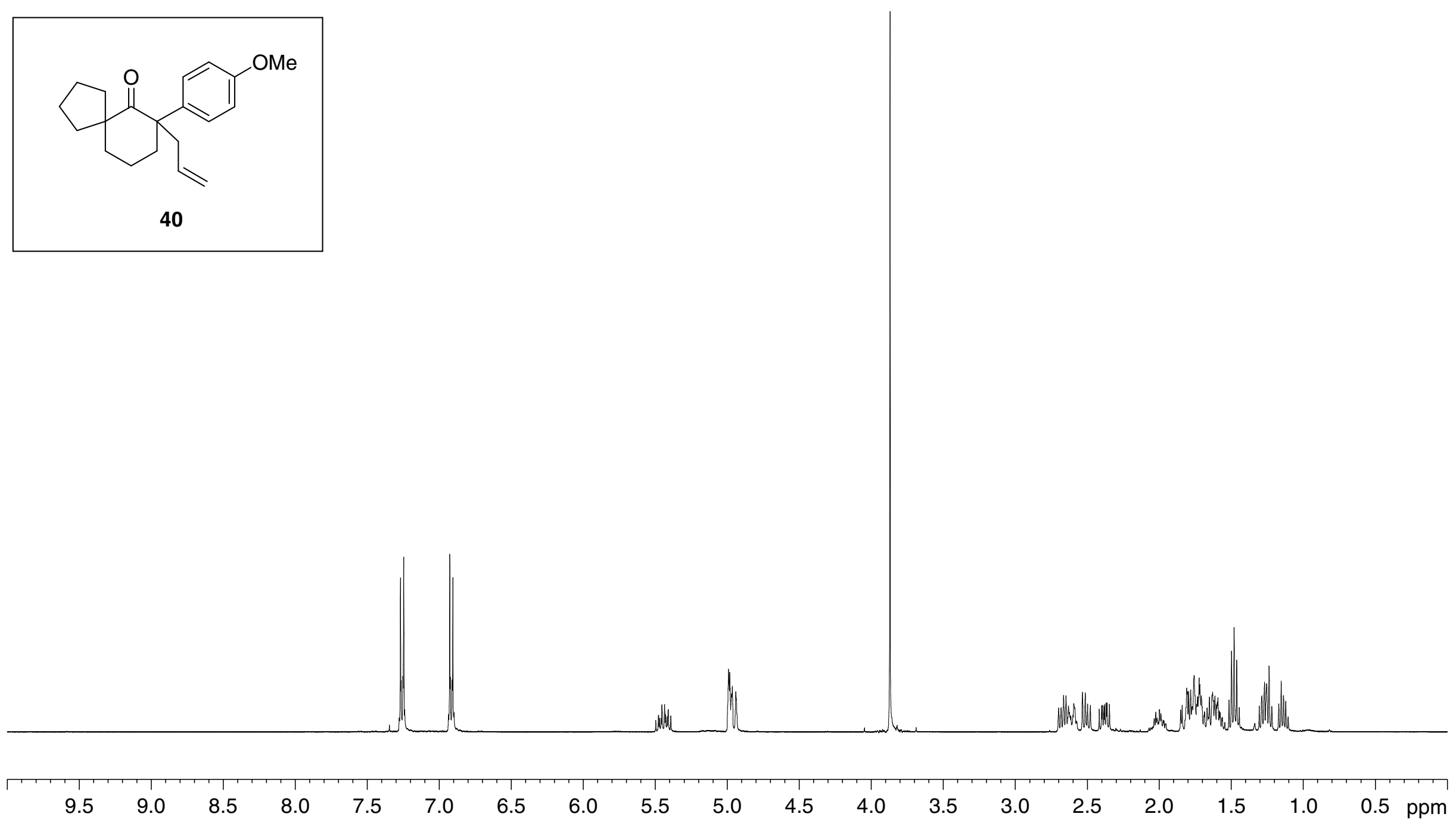
${ }^{13}$ C NMR (JMOD) Spectrum of 40; Solvent: $\mathrm{CDCl}_{3} ; 100.61 \mathrm{MHz}$; Instrument: Bruker DPX400 Spectrometer
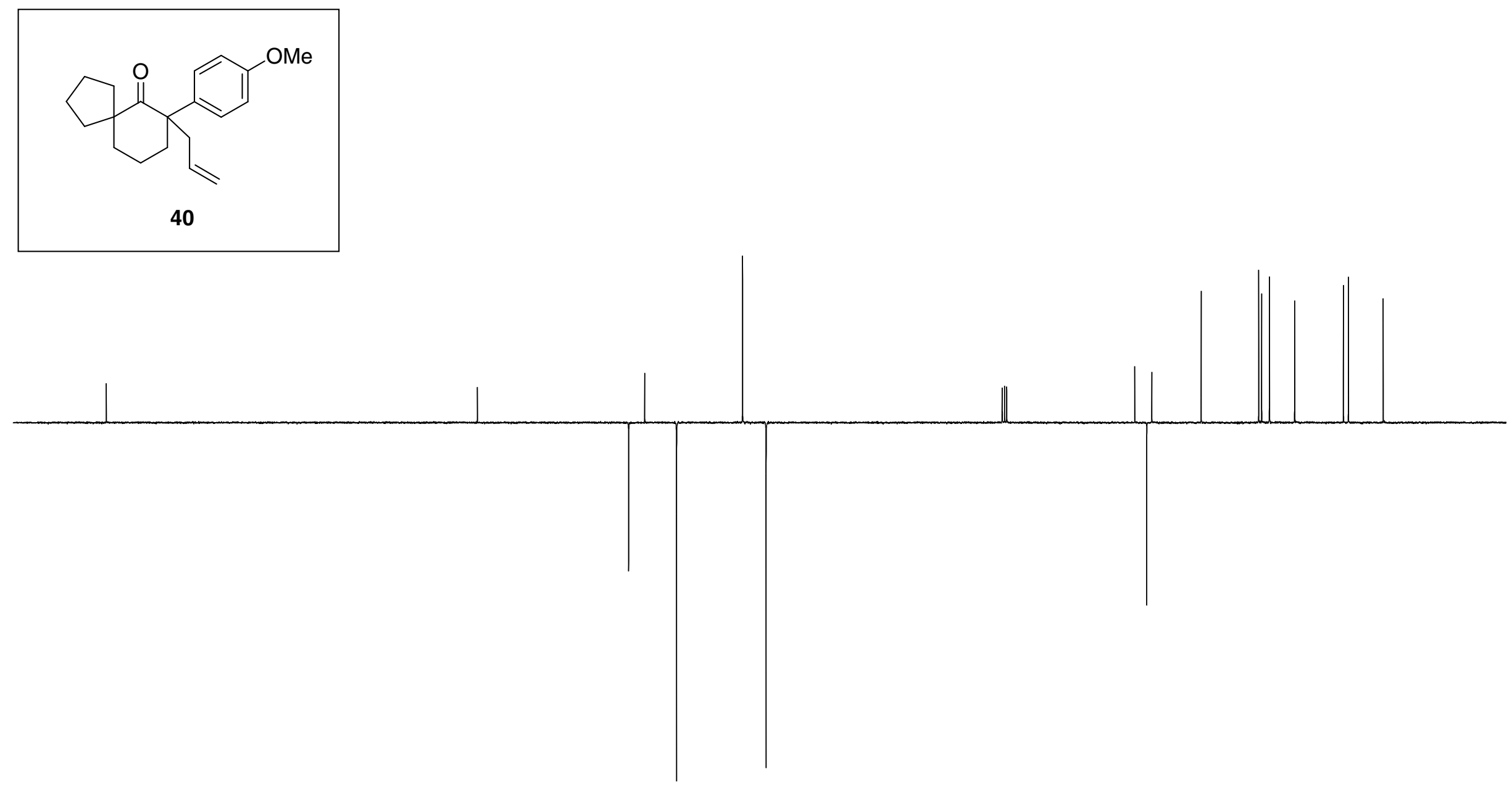

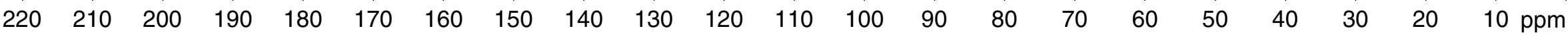


${ }^{1} \mathrm{H}$ NMR Spectrum of 41; Solvent: $\mathrm{CDCl}_{3} ; 400.13 \mathrm{MHz}$; Instrument: Bruker DPX400 Spectrometer
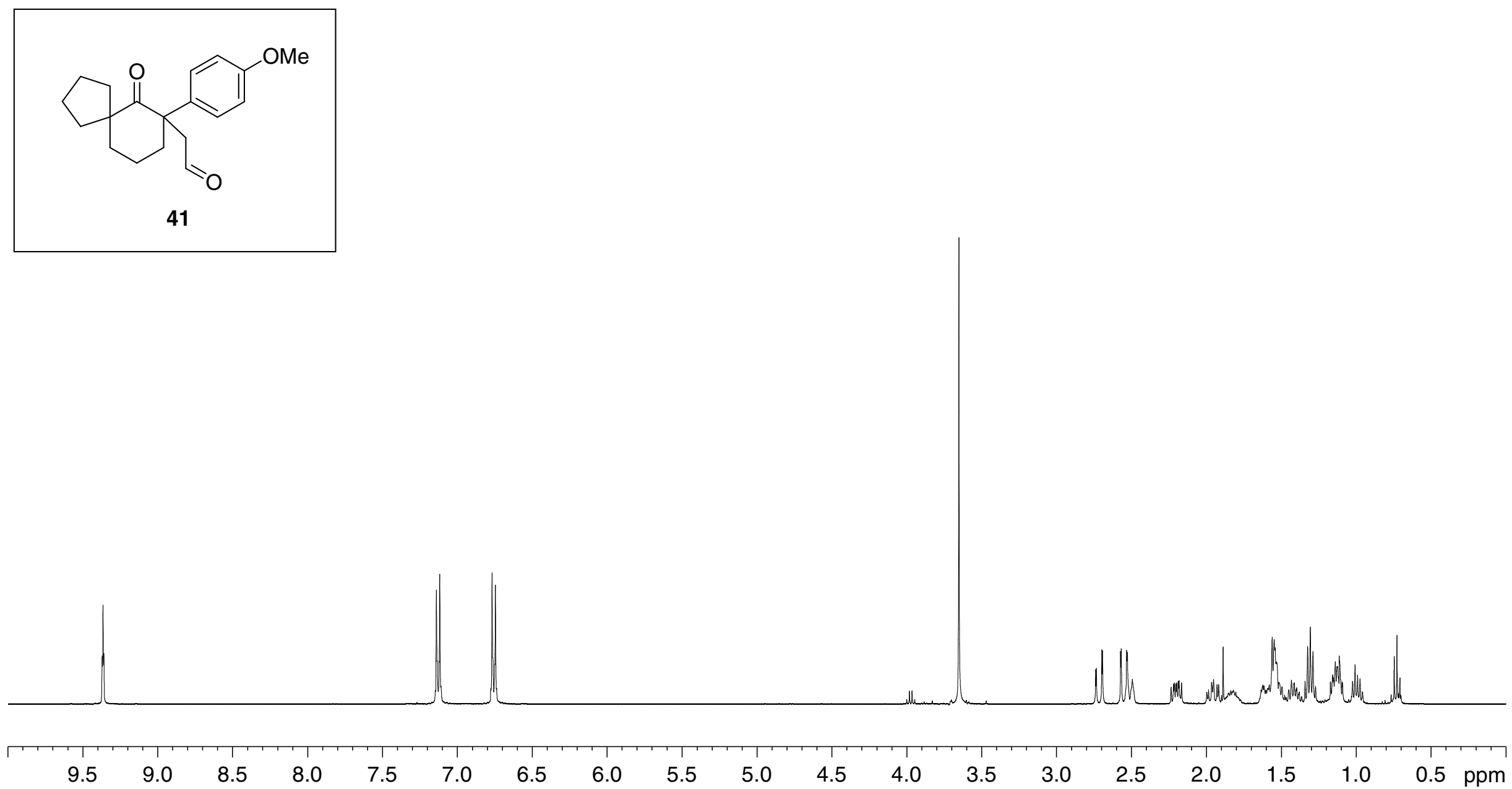
${ }^{13}$ C NMR (JMOD) Spectrum of 41; Solvent: $\mathrm{CDCl}_{3} ; 100.61 \mathrm{MHz}$; Instrument: Bruker DPX400 Spectrometer
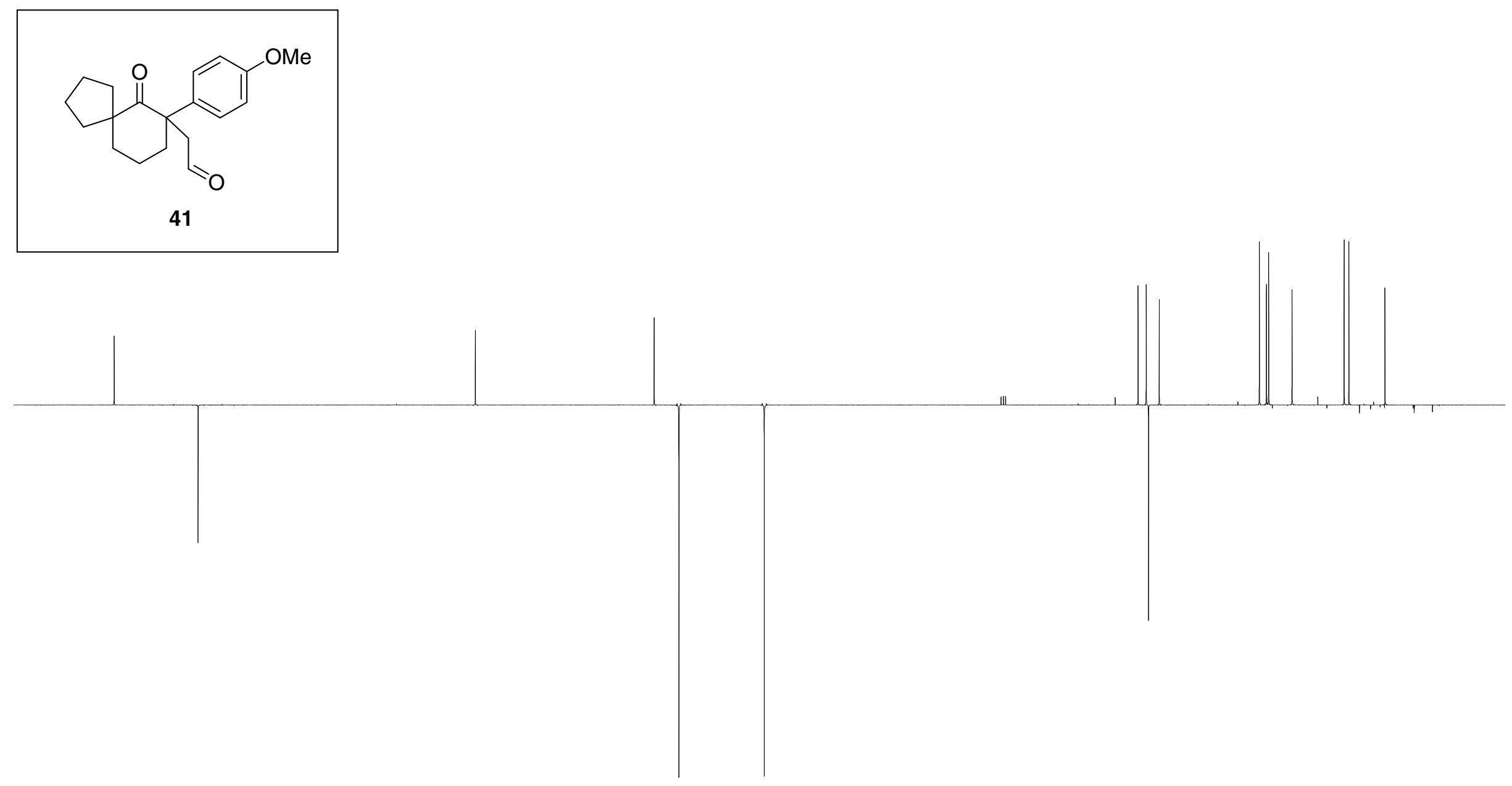
${ }^{1} \mathrm{H}$ NMR Spectrum of 43; Solvent: $\mathrm{CDCl}_{3}$; $400.13 \mathrm{MHz}$; Instrument: Bruker DPX400 Spectrometer
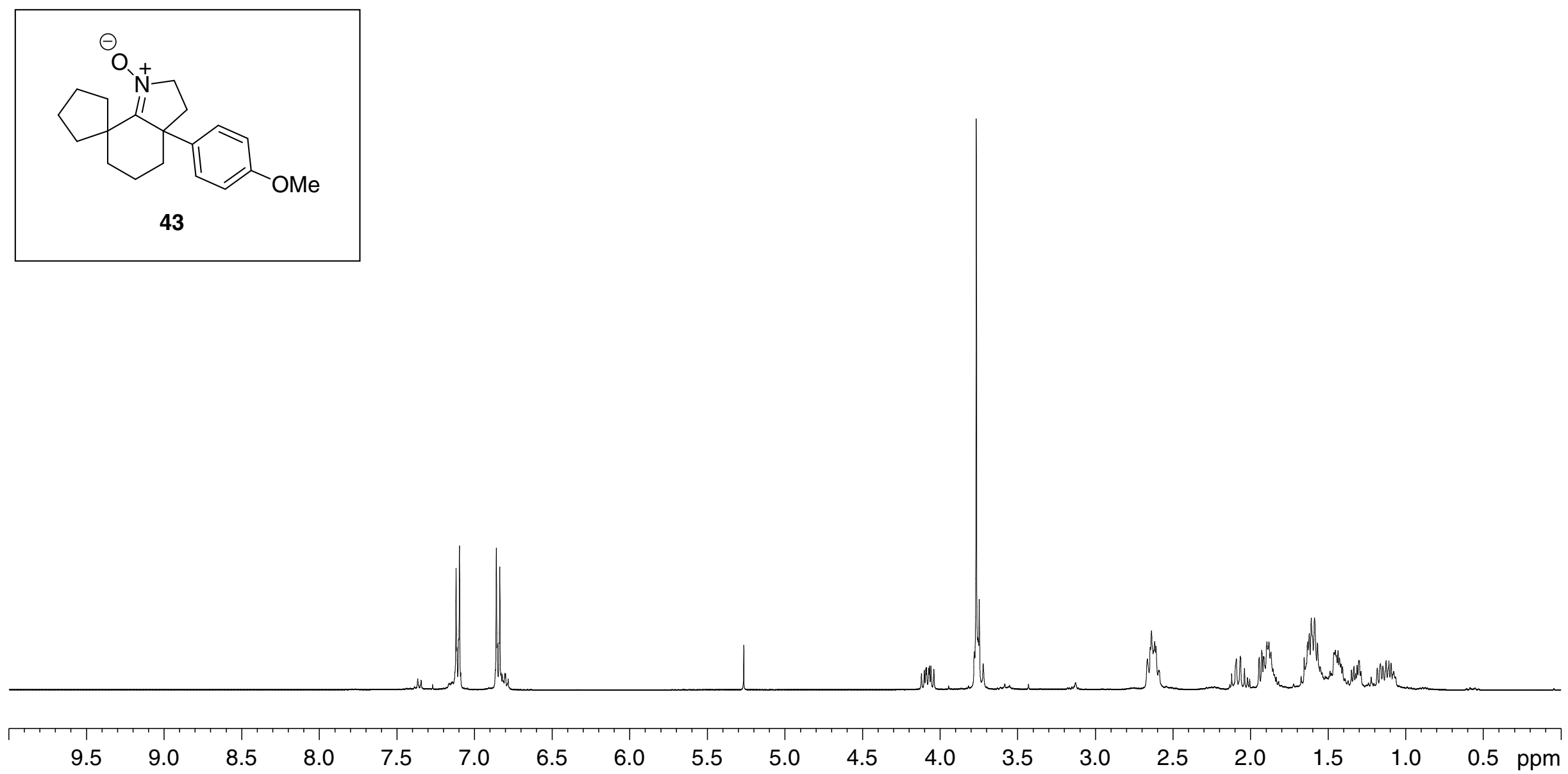
${ }^{13} \mathrm{C}$ NMR (JMOD) Spectrum of 43; Solvent: $\mathrm{CDCl}_{3} ; 100.61 \mathrm{MHz}$; Instrument: Bruker DPX400 Spectrometer

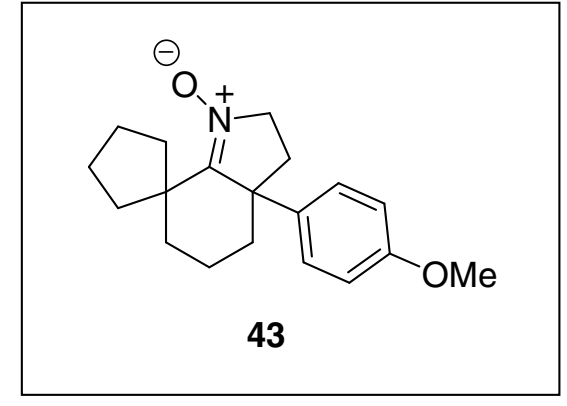

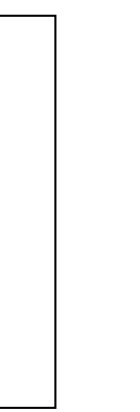
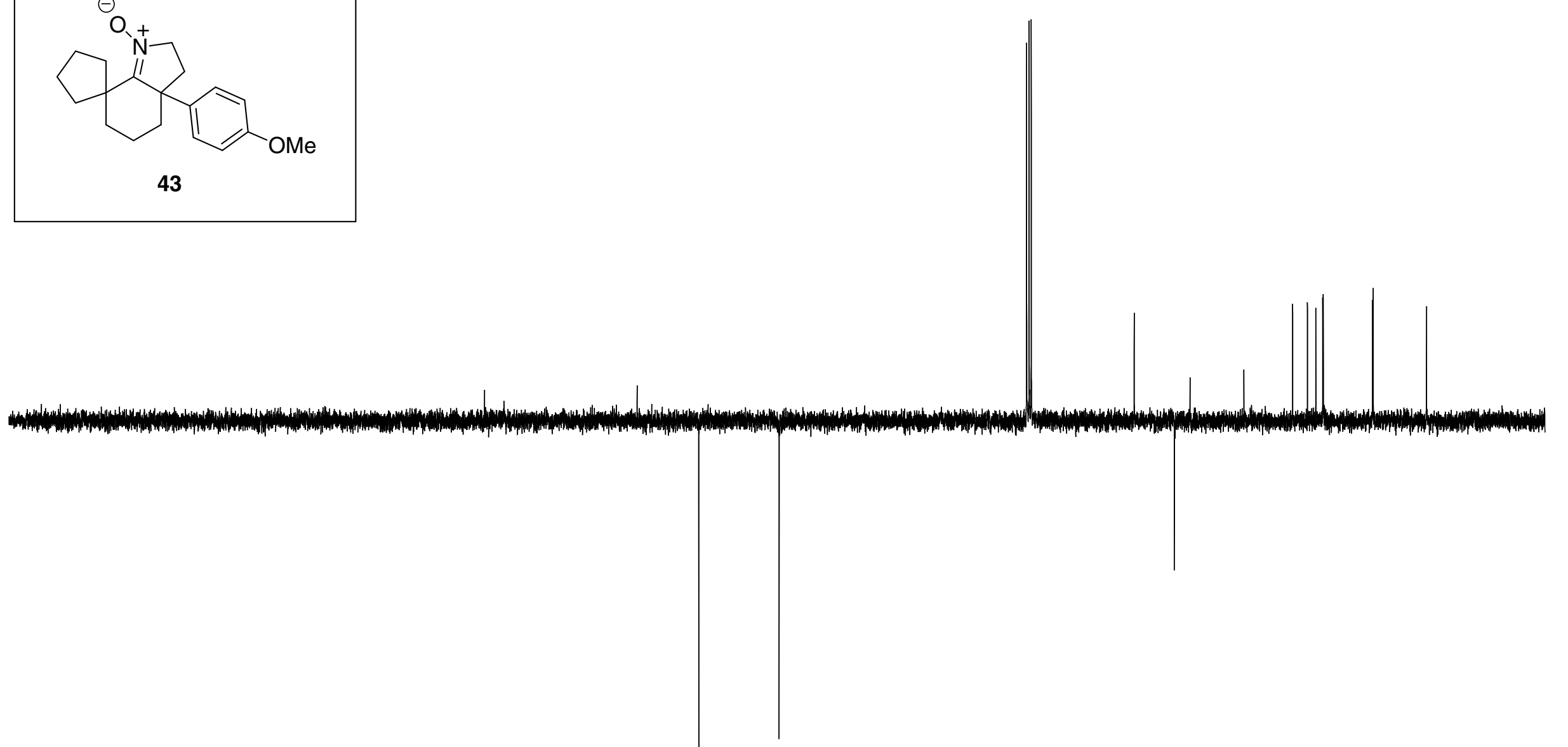

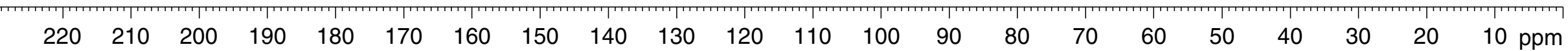


${ }^{1} \mathrm{H}$ NMR Spectrum of 44; Solvent: $\mathrm{CDCl}_{3} ; 400.13 \mathrm{MHz}$; Instrument: Bruker DPX400 Spectrometer
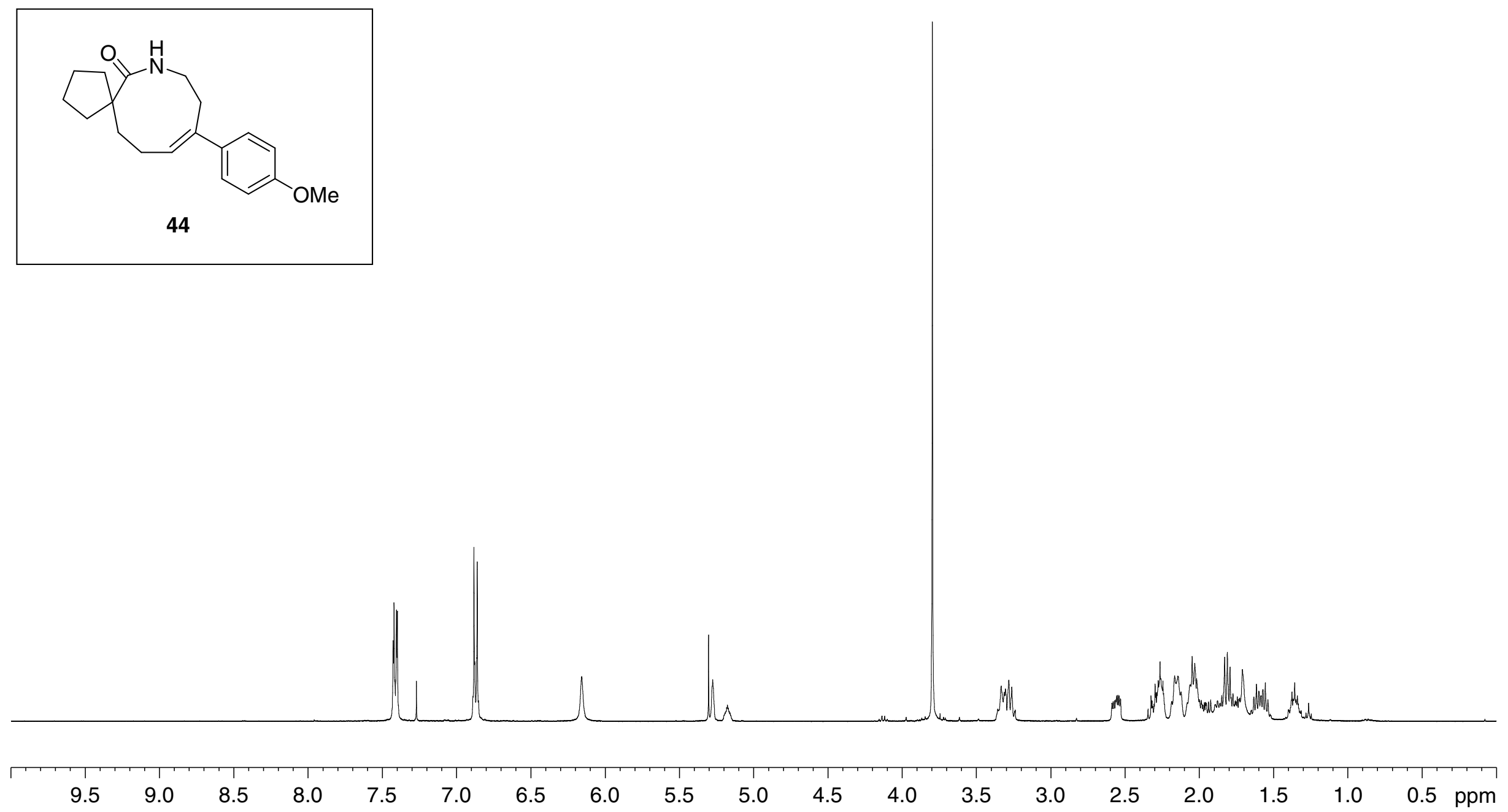
${ }^{13} \mathrm{C}$ NMR (JMOD) Spectrum of 43; Solvent: $\mathrm{CDCl}_{3} ; 100.61 \mathrm{MHz}$; Instrument: Bruker DPX400 Spectrometer
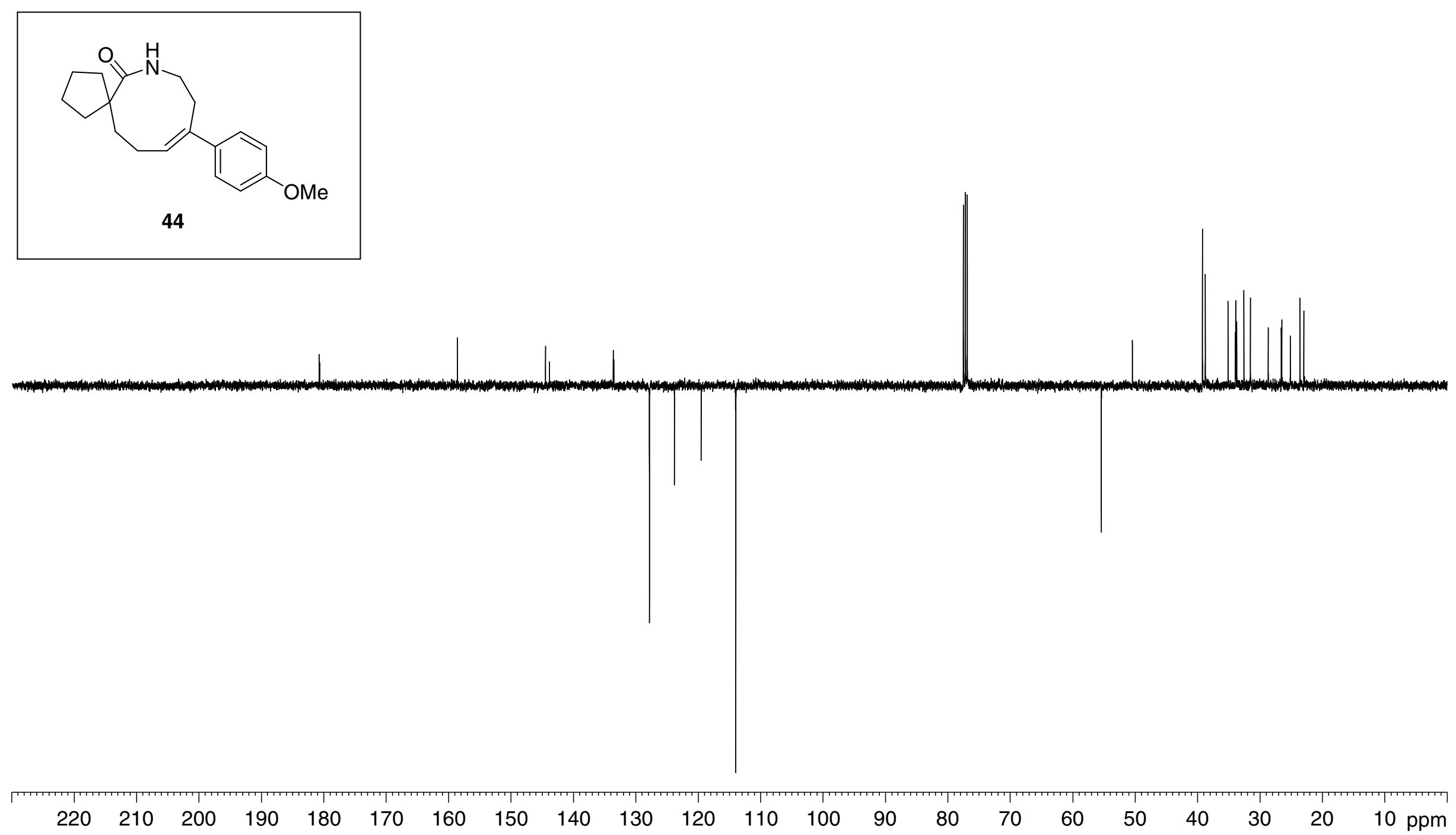


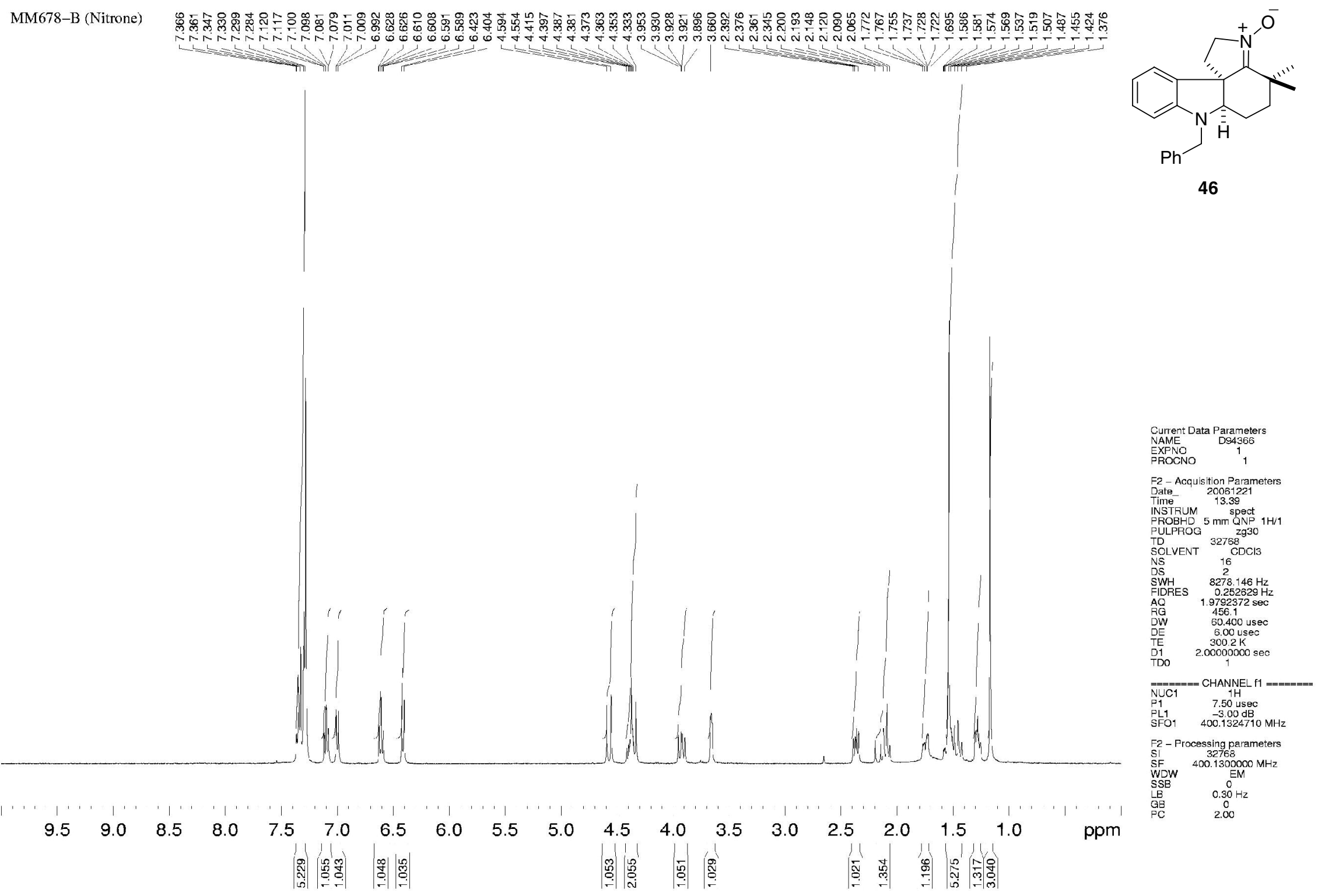



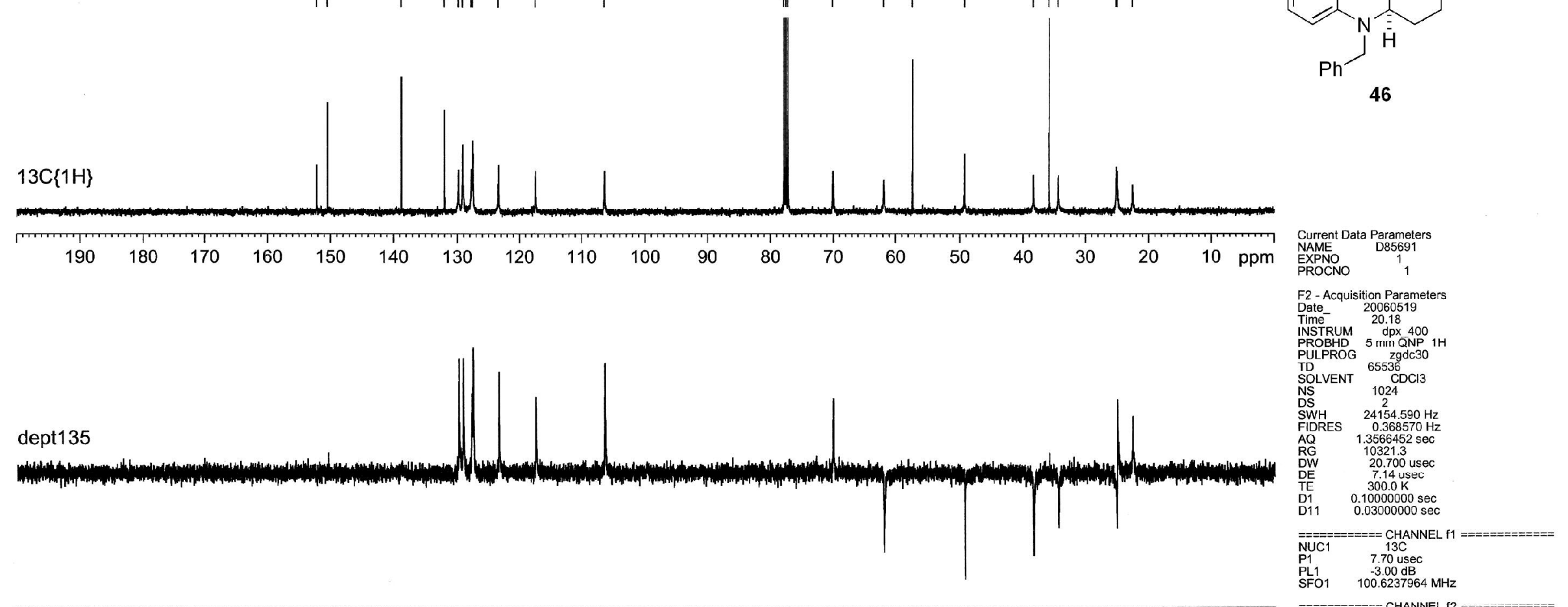

\section{dept90}

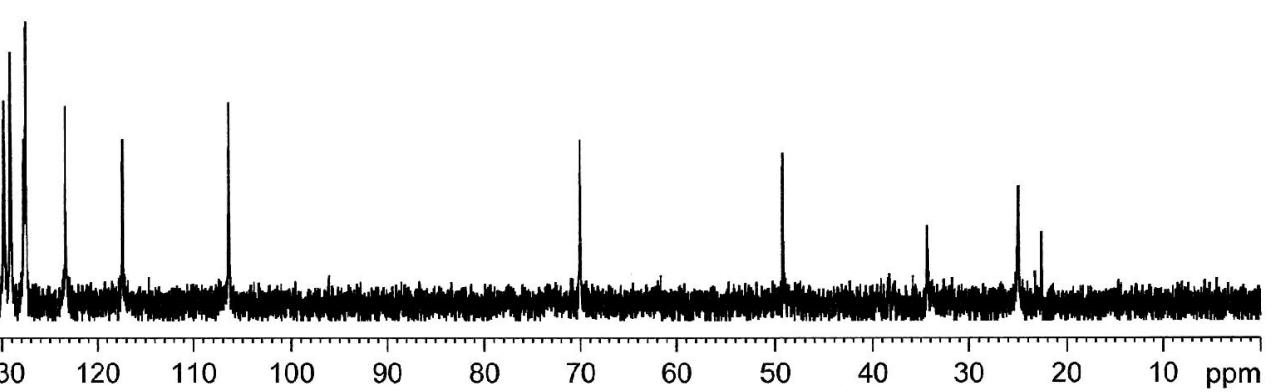




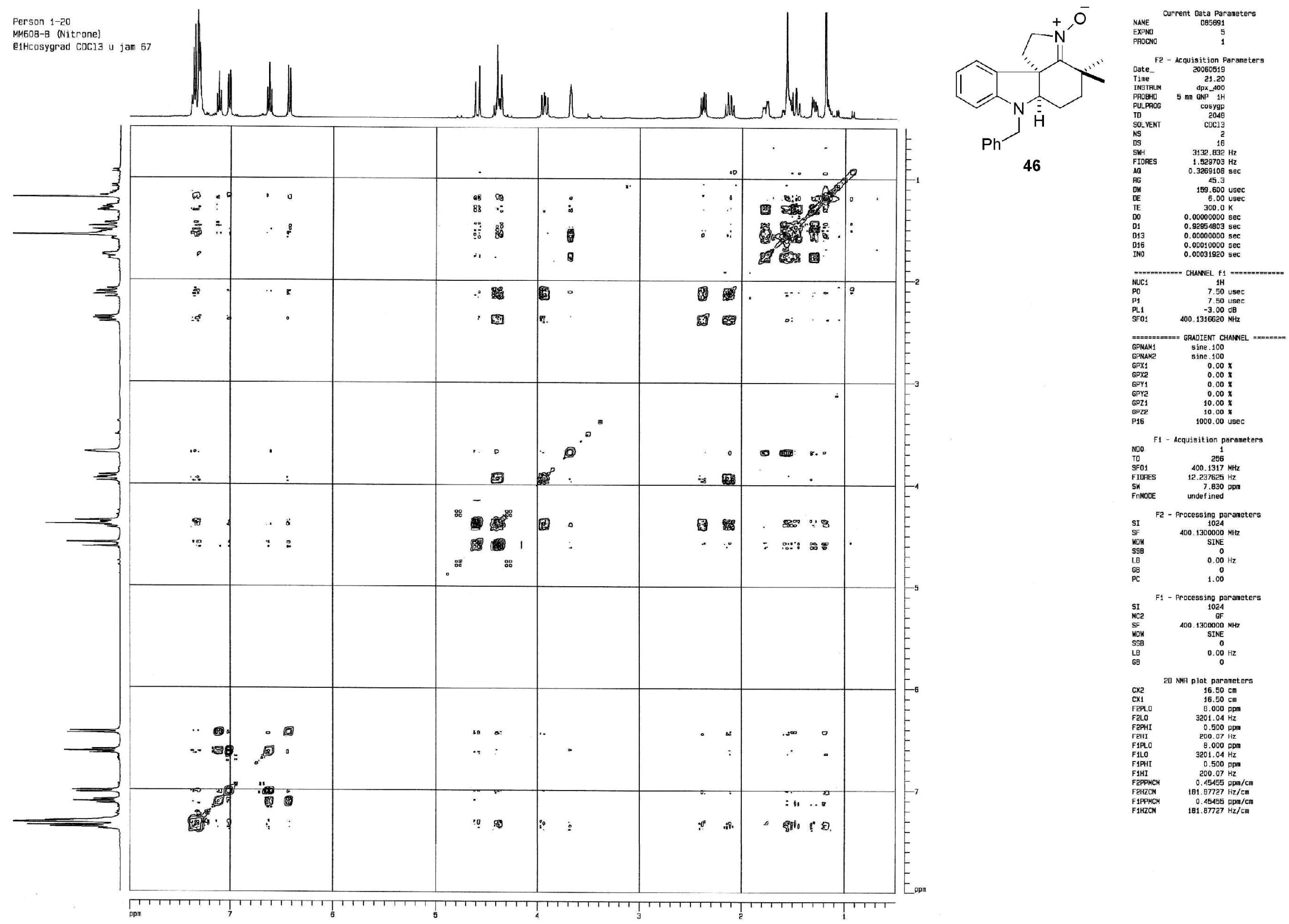




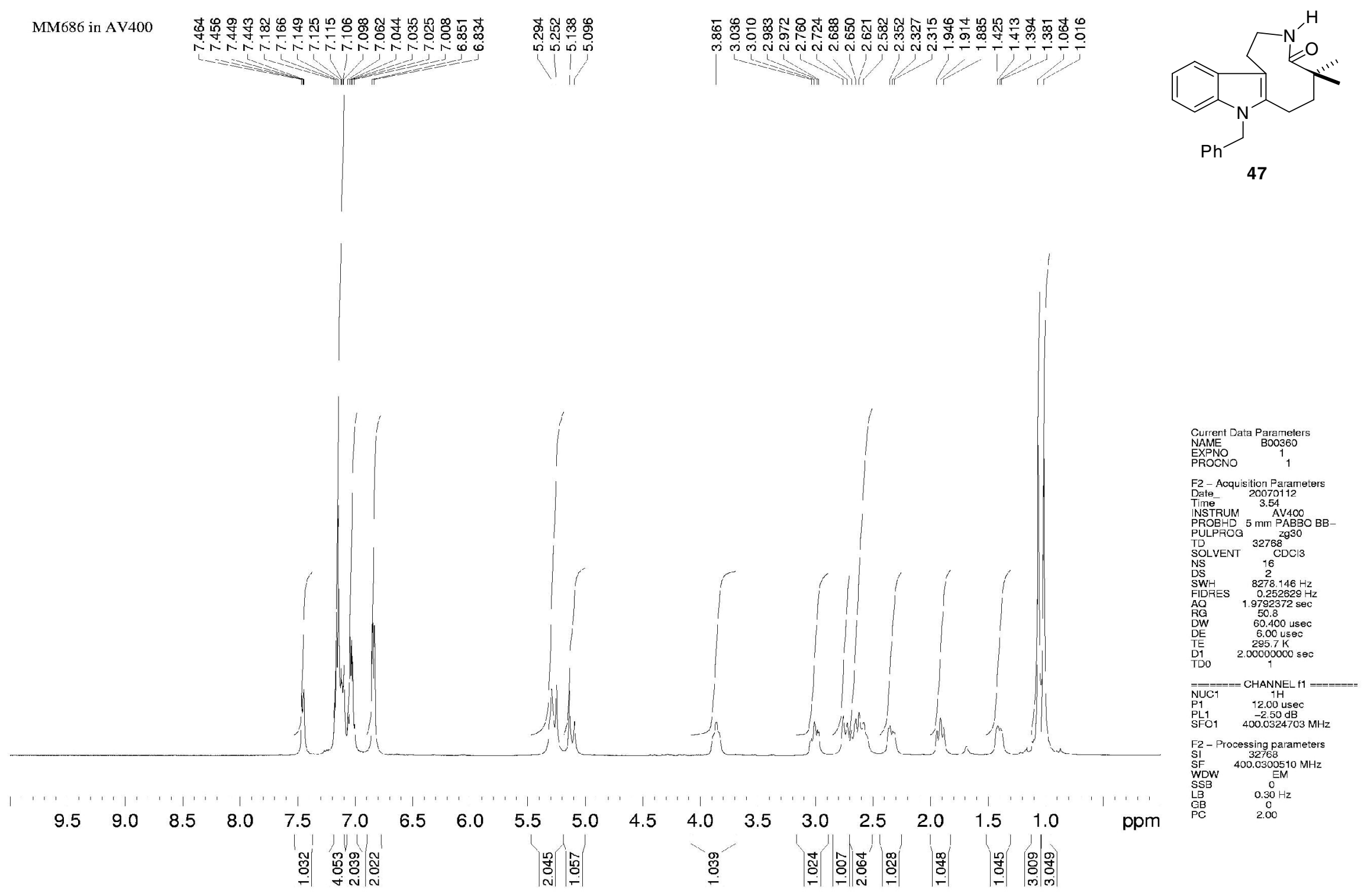




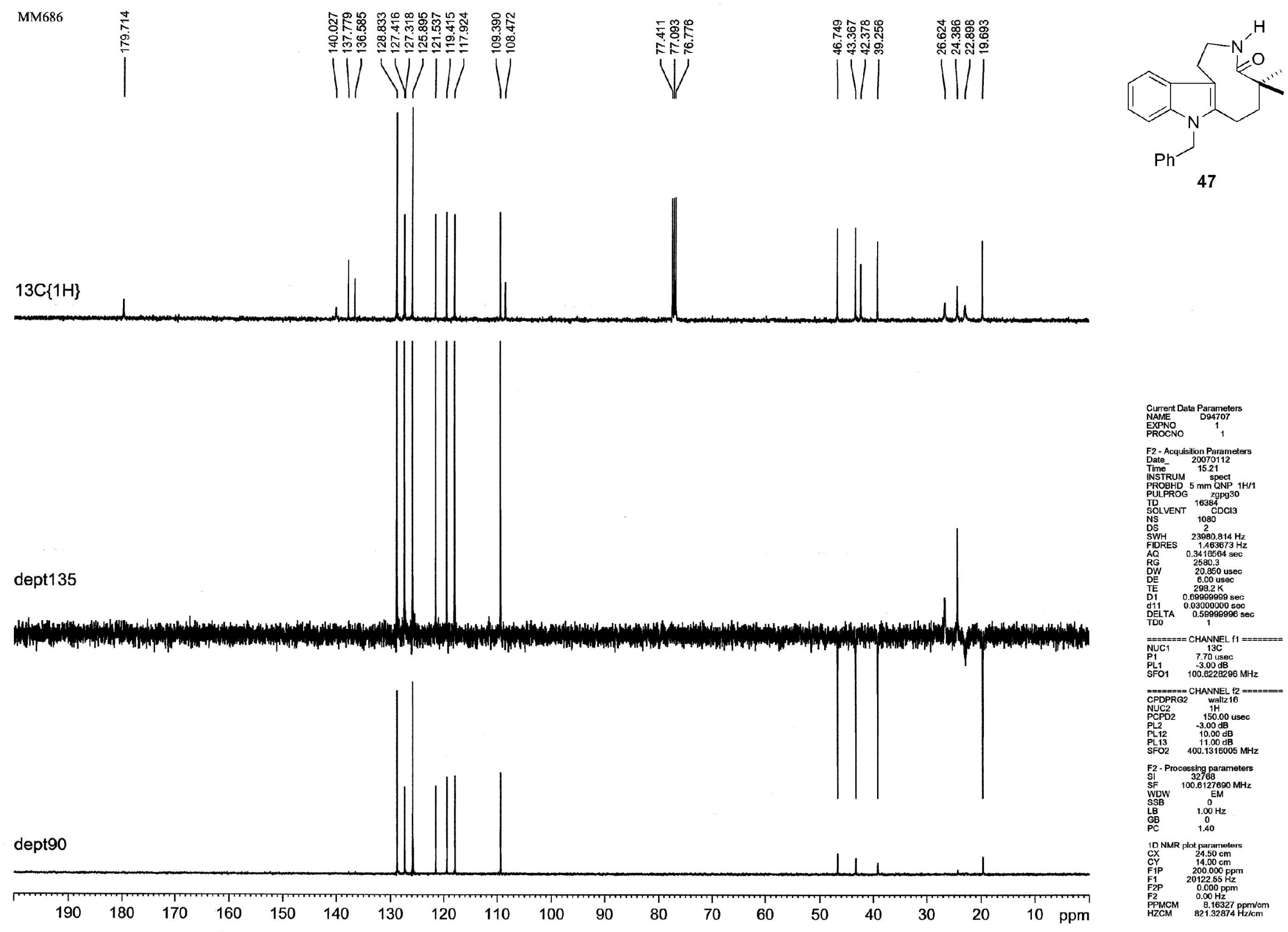


2D ${ }^{1} \mathrm{H}-{ }^{1} \mathrm{H}$ COSY NMR Spectrum of Compound

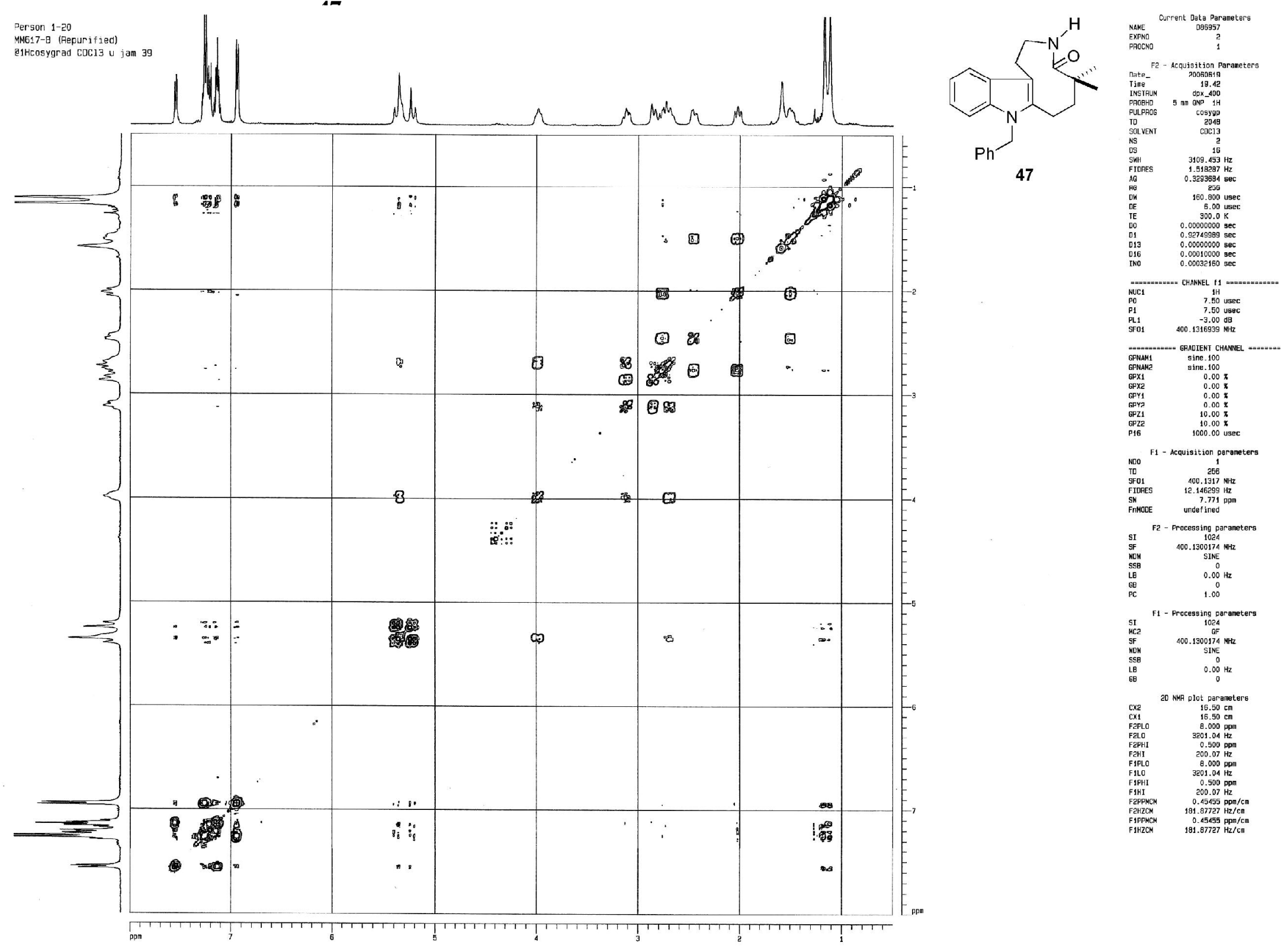




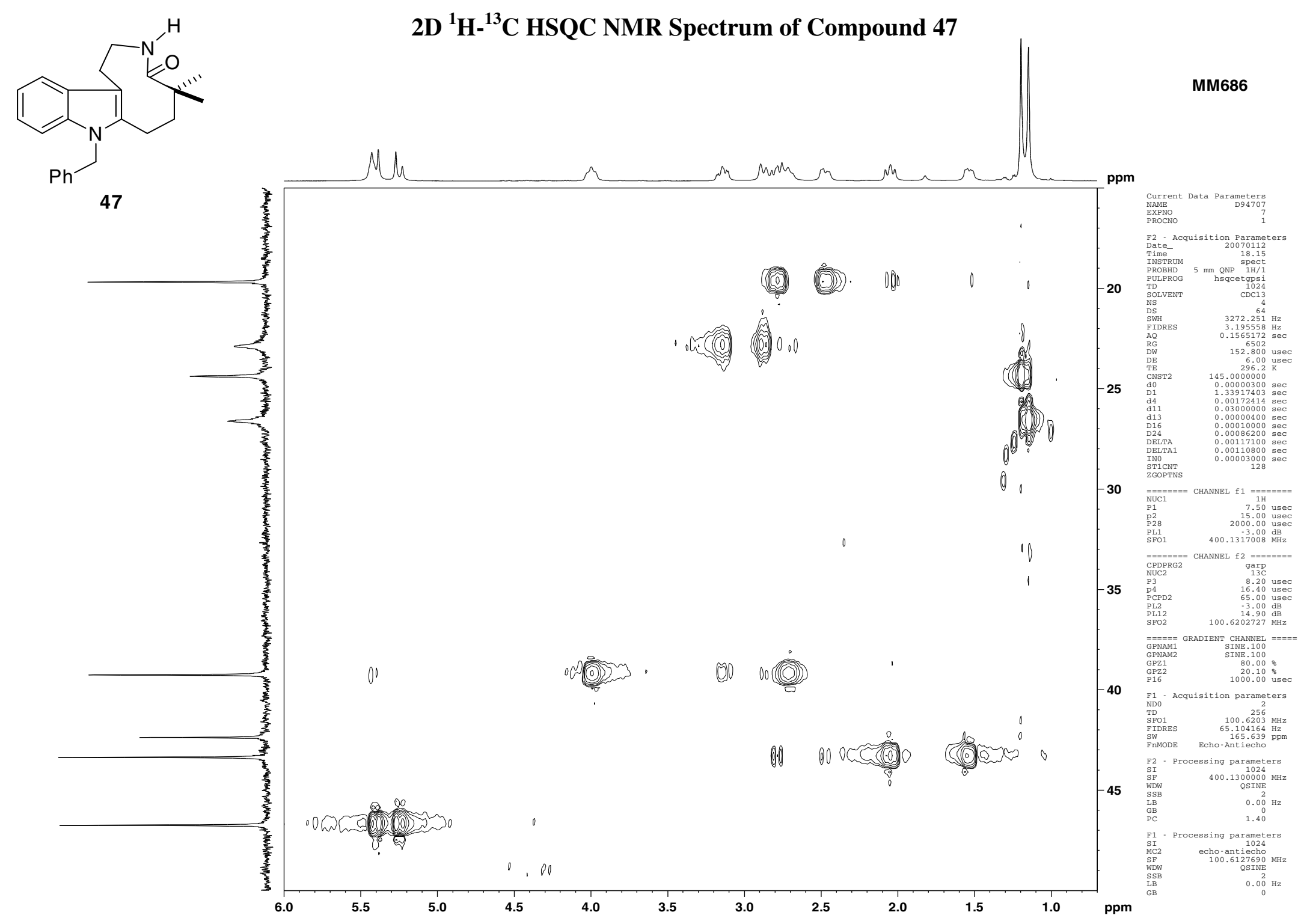

